\title{
Higgs interference effects at the one-loop level in the 1-Higgs-Singlet extension of the Standard Model
}

\author{
Nikolas Kauer, ${ }^{a}$ Alexander Lind, ${ }^{a, b}$ Philipp Maierhöfer ${ }^{c}$ and Weimin Song ${ }^{d}$ \\ ${ }^{a}$ Department of Physics, Royal Holloway, University of London, \\ Egham Hill, Egham TW20 0EX, U.K. \\ ${ }^{b}$ Department of Physics and Astronomy, University of Sussex, \\ Brighton BN1 9QH, U.K. \\ ${ }^{c}$ Physikalisches Institut, Albert-Ludwigs-Universität Freiburg, \\ 79104 Freiburg, Germany \\ ${ }^{d}$ Rutherford Appleton Laboratory, \\ Harwell Oxford, Didcot OX11 OQX, U.K. \\ E-mail: n.kauer@rhul.ac.uk, alexander.lind.2017@live.rhul.ac.uk, \\ philipp.maierhoefer@physik.uni-freiburg.de, weimin.song@cern.ch
}

Abstract: A detailed study of Higgs interference effects at the one-loop level in the 1Higgs-Singlet extension of the Standard Model (1HSM) is presented for the $W^{+} W^{-}$and $t \bar{t}$ decay modes with fully leptonic $W W$ decay. We explore interference effects for benchmark points with a heavy Higgs mass that significantly exceeds $2 m_{t}$. In the $W W$ channel, the Higgs signal and the interfering continuum background are loop induced. In the $t \bar{t}$ channel, which features a tree-level background, we also calculate the interference with the one-loop background, which, due to the appearance of the absorptive part, is found to dominate the normalisation and shape of differential Higgs cross section distributions and should therefore be considered in experimental analyses. The commonly used geometric average $K$-factor approximation $K_{\text {interference }} \approx\left(K_{\text {Higgs }} K_{\text {background }}\right)^{1 / 2}$ is not appropriate. We calculate with massive top and bottom quarks. Our 1HSM and SM implementation in SHERPA+OPEnLoOPs is publicly available and can be used as parton-level integrator or event generator.

Keywords: Higgs Physics, Beyond Standard Model, Perturbative QCD

ArXiv EPrint: 1905.03296 


\section{Contents}

1 Introduction 1

2 Model 4

$\begin{array}{lll}3 & \text { Calculational details } & 6\end{array}$

$\begin{array}{lll}4 & \text { Results } & 9\end{array}$

$\begin{array}{llr}5 & \text { Discussion } & 27\end{array}$

$\begin{array}{llr}6 & \text { Conclusions } & 29\end{array}$

A Partial decay widths for $h_{2} \rightarrow h_{1} h_{1}, h_{1} h_{1} h_{1}, h_{1} h_{1} h_{1} h_{1}$

$\begin{array}{ll}\text { B Nonredundant complete set of integrated results } & 31\end{array}$

C Standard Model distributions $\quad 33$

\section{Introduction}

In 2012, the ATLAS and CMS experiments at the CERN Large Hadron Collider (LHC) announced the discovery of a new scalar resonance with a mass of approximately $125 \mathrm{GeV}[1,2]$. The discovered particle is so far consistent with the Higgs boson predicted by the Standard Model (SM) Higgs mechanism [3-7], but many extensions to the SM preserve the minimal assumptions of an $\mathrm{SU}(2)$ doublet which acquires a vacuum expectation value thus inducing a physical Higgs boson that couples to fermions and vector bosons in proportion to their mass, while also allowing for an expanded Higgs sector with additional, heavier Higgs-like scalar particles.

Since a SM-like Higgs boson has been discovered, a theoretically consistent search for an additional Higgs boson has to be based on a model that is beyond the SM (BSM). The simplest extension of the Higgs sector of the SM introduces an additional real scalar singlet field which is neutral under the SM gauge groups. This 1-Higgs-Singlet extension of the SM, abbreviated as 1HSM, has been extensively explored in the literature [8-38]. The remaining viable parameter space of the 1HSM after LHC Run 1 has been studied in refs. [39-42].

At the LHC, ATLAS and CMS have been conducting searches for heavier Higgs-like bosons in various di-boson channels, in particular $W^{+} W^{-}$[43-49], and in various di-fermion channels, in particular $t \bar{t}[50,51]$.

So far, the heavy Higgs searches are geared to establishing a significant excess ("bump") in the invariant mass spectrum of the final state particles at the position of the heavy 


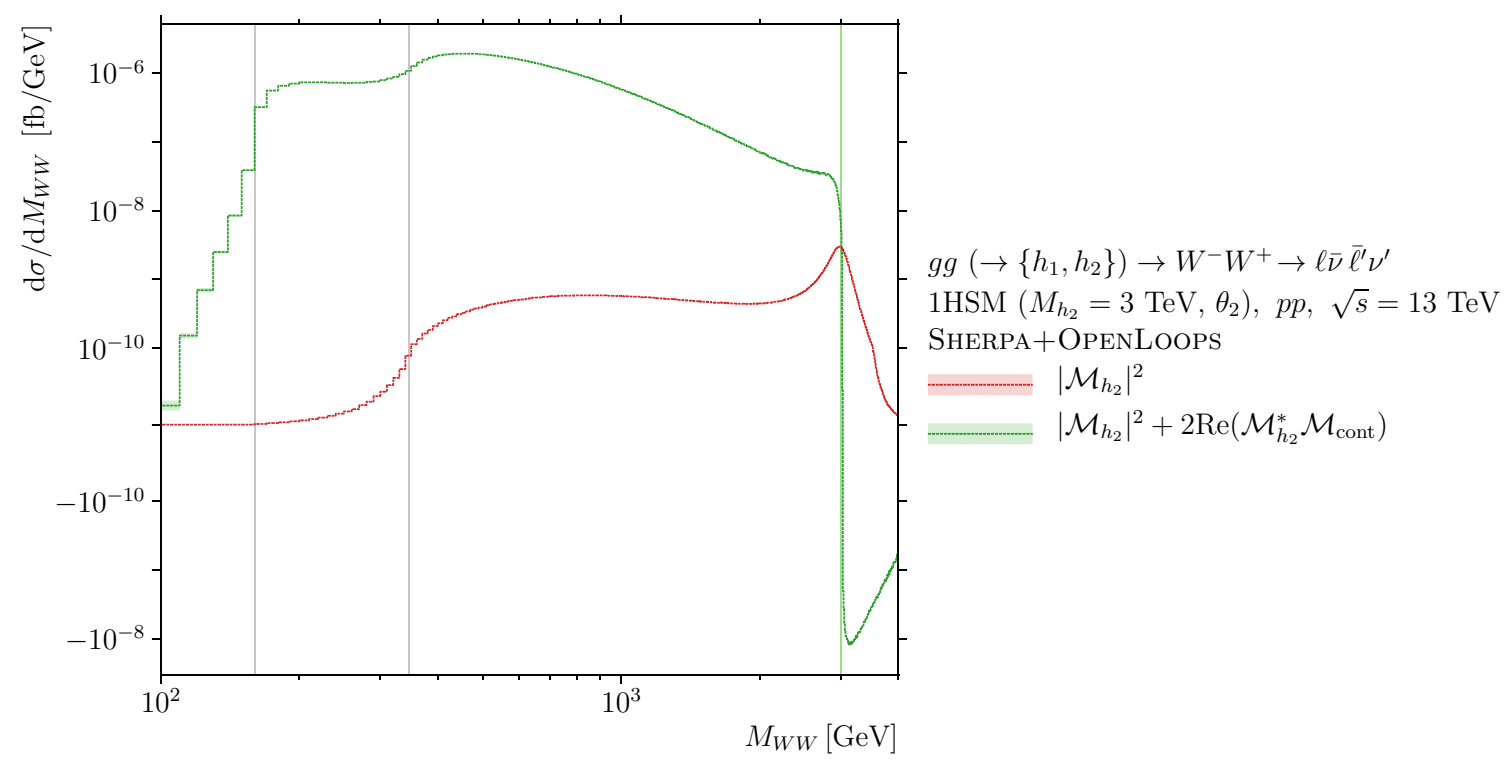

Figure 1. $M_{W W}$ distribution of the heavy Higgs cross section and including its interference with the continuum background in the 1-Higgs-Singlet Extension of the SM (1HSM) with $M_{h_{2}}=3 \mathrm{TeV}$ and mixing angle $\theta_{2}$ (see table 1) for $g g\left(\rightarrow\left\{h_{1}, h_{2}\right\}\right) \rightarrow W^{-} W^{+} \rightarrow \ell \bar{\nu} \bar{\ell}^{\prime} \nu^{\prime}$ in $p p$ collisions at $\sqrt{s}=13 \mathrm{TeV}$. For details, see sections 2 and 4 .

resonance. However, as illustrated in figure 1, the "bump" of the heavy resonance can turn out to be a tiny correction to the heavy resonance signal when signal-background interference is taken into account. Note also that the line shape of the resonance without and with interference has no resemblance. ${ }^{1}$ We emphasise that the signal-background interference is a constituent of the BSM signal and a priori has to be treated on equal footing with the mod-squared BSM amplitude (the "bump"). If the BSM amplitude is absent, the interference vanishes. It is therefore crucial to calculate and study interference effects for heavy resonance searches. Furthermore, as demonstrated below, in general it is crucial to take one-loop corrections to tree-level amplitudes into account to obtain reliable predictions.

Here, we focus on the case where the additional Higgs boson is heavier than the discovered Higgs boson. In this case, the BSM signal is affected not only by a sizeable Higgs

\footnotetext{
${ }^{1}$ For $\sigma\left(\left|\mathcal{M}_{h_{2}}\right|^{2}\right)$, figure 1 shows a shoulder extending from below the heavy Higgs resonance down to $2 m_{t}$. This significant deviation from the expected Breit-Wigner shape results from the convolution with the strongly rising (for $M_{W W} \rightarrow 0$ ) gluon parton distribution function (PDF). We note that the shoulder does not effectuate an enhanced experimental sensitivity to the heavy Higgs signal. This is apparent from the results given in section 4 and appendix B and can be understood qualitatively as follows. Before convolution with the PDF, for the continuum background cross section $d \sigma_{\text {cont }} \sim \hat{s}^{-1}$ (up to powers of $\log \hat{s})$. For $\sqrt{\hat{s}} \gg M_{\text {Higgs }}$, the same behaviour applies to $d \sigma_{\text {Higgs }}$. However, in the invariant mass region significantly below the Higgs resonance, one has $1 /\left(\hat{s}-M_{\text {Higgs }}^{2}\right)^{2} \sim M_{\text {Higgs }}^{-4}$, rather than $\hat{s}^{-2}$. This changes the dependence to $d \sigma_{\text {Higgs }} \sim \hat{s}$. In this region, for decreasing $\sqrt{\hat{s}}, d \sigma_{\text {Higgs }}$ decreases while $d \sigma_{\text {cont }}$ increases. The background hence outgrows the Higgs cross section when moving further and further below $M_{\text {Higgs }}$. Convolution with the PDF does not affect this relative change.
} 
interference with the continuum background, but also by a non-negligible interference between the heavy Higgs boson and the high-mass tail of the light Higgs boson [52], which is fully taken into account in the calculations presented here.

For the $W W$ and $Z Z$ decay modes, interference effects in 2-Higgs models have been studied previously. In gluon fusion Higgs production, the heavy Higgs-light Higgs interference was studied in the 1HSM in refs. [53-55] and in 2-Higgs-doublet models (2HDMs) in ref. [56]. ${ }^{2}$

The peak-dip deformation of the Higgs resonance in $g g \rightarrow t \bar{t}$ due to signal-background interference was first studied in the SM in ref. [60]. It was further studied for heavy scalars in 2-Higgs models for masses up to $750 \mathrm{GeV}$ in ref. [61, 62]. ${ }^{3}$ A detailed analytic discussion and illustrative study of the heavy Higgs line shape modification due to signal background interference in $g g \rightarrow t \bar{t}$ for scalar masses up to $1 \mathrm{TeV}$ was presented in ref. [64]. ${ }^{4}$ Recently, a detailed study of the experimental sensitivity to additional heavy (pseudo)scalar resonances with mass up to $1 \mathrm{TeV}$ in the singlet model, 2HDM and the hMSSM in $g g \rightarrow t \bar{t}$ at the LHC, taking into account signal-background interference effects, was presented in ref. [65]. In this paper, we extend the work of ref. [65] by studying the Higgs signal in the $0.7 \mathrm{TeV}$ to $3 \mathrm{TeV}$ mass range for integrated cross sections and differential distributions in $M_{t \bar{t}}$ and various kinematic observables. Interference effects between all three $g g \rightarrow t \bar{t}$ amplitude contributions - heavy Higgs, continuum background and light Higgs - are taken into account and illustrated individually. Significantly, we investigate the impact of higher-order corrections on the Higgs signal by accurately taking into account its interference with the virtual corrections to the $g g \rightarrow t \bar{t}$ continuum background. ${ }^{5}$ Due to a non-trivial phase, loop-level amplitude contributions can substantially change integrated cross sections and the shapes of differential cross sections [66-68]. Furthermore, it is wellknown that "flat" inclusive $K$-factors often do not model differential NLO corrections well. In 2HDMs, signal-background interference effects have also been studied in the context of heavy Higgs searches in the $t b W$ final state [69]. NLO effects in effective field theory fits to $W^{+} W^{-}$production at the LHC have been studied in ref. [70] and the implementation is publicly available through the POWHEG-BOX.

This paper is organised as follows: in section 2 we discuss the 1HSM and specify the used benchmark points. In section 3 we review the details of our calculation and specify the used input parameters and settings. In section 4 , we present cross sections and distributions

\footnotetext{
${ }^{2}$ A calculation including full interference effects in a Higgs portal model has been carried out in ref. [57]. For Higgs production in vector boson fusion, heavy-light interference in a 2-Higgs model was studied in ref. [58] for an $e^{+} e^{-}$collider and in more detail including heavy-continuum interference in ref. [59] for the LHC.

${ }^{3}$ See also ref. [63].

${ }^{4}$ Loop corrections to the background are not considered in ref. [64].

${ }^{5}$ In ref. [65], the interference is calculated at leading order (LO) and rescaled with the geometric average of inclusive $K$-factors for the signal and QCD background in an attempt to approximate higher-order corrections. This approach was also used in ref. [62] to obtain approximate next-to-leading order (NLO) results for heavy scalar $\left(h_{2}\right)$ production in $g g \rightarrow t \bar{t}+$ jet with $M_{h_{2}}=500 \mathrm{GeV}$. In ref. [61], for the $2 \mathrm{HDM}$ approximate NLO corrections were calculated using the effective $g g(g) H$ vertices obtained in the heavy top quark limit.
} 
for the Higgs signal and its interference in the 1HSM and, for comparison, in the SM for $g g \rightarrow$ Higgs $\rightarrow W W$ and $t \bar{t}$ with fully leptonic $W W$ decay taking into account tree- and one-loop backgrounds. In section 5 , we discuss our findings. We conclude in section 6 .

\section{Model}

As a minimal theoretically consistent model with two physical Higgs bosons, we consider the 1HSM, i.e. the SM with an added real singlet field which is neutral under all SM gauge groups. ${ }^{6}$ In the following, we give a brief summary of the model. A more detailed description can be found in refs. [29, 71].

The SM Higgs sector is extended by the addition of a new real scalar field, which is a singlet under all the gauge groups of the SM and which also gets a vacuum expectation value (VEV) under electroweak symmetry breaking. The most general gauge-invariant potential can be written as $[9,11]$

$$
V=\lambda\left(\Phi^{\dagger} \Phi-\frac{v^{2}}{2}\right)^{2}+\frac{1}{2} M^{2} s^{2}+\lambda_{1} s^{4}+\lambda_{2} s^{2}\left(\Phi^{\dagger} \Phi-\frac{v^{2}}{2}\right)+\mu_{1} s^{3}+\mu_{2} s\left(\Phi^{\dagger} \Phi-\frac{v^{2}}{2}\right),
$$

where $s$ is the real singlet scalar which is allowed to mix with the SM SU(2) Higgs doublet, which in the unitary gauge can be written as

$$
\Phi=\left(\begin{array}{c}
0 \\
(\phi+v) / \sqrt{2}
\end{array}\right)
$$

with VEV $v \simeq 246 \mathrm{GeV}$. Here it has already been exploited that (without the $Z_{2}$ symmetry) shifting the singlet field simply corresponds to a redefinition of the parameter coefficients and due to this freedom one can take the VEV of the singlet field to zero, which implies $M^{2}>0$. To avoid vacuum instability the quartic couplings must satisfy

$$
\lambda>0, \quad \lambda_{1}>0, \quad \lambda_{2}>-2 \sqrt{\lambda \lambda_{1}} .
$$

The trilinear couplings $\mu_{1}$ and $\mu_{2}$ can have positive or negative sign. Substituting eq. (2.2) into eq. (2.1), one obtains the potential

$$
V=\frac{\lambda}{4} \phi^{4}+\lambda v^{2} \phi^{2}+\lambda v \phi^{3}+\frac{1}{2} M^{2} s^{2}+\lambda_{1} s^{4}+\frac{\lambda_{2}}{2} \phi^{2} s^{2}+\lambda_{2} v \phi s^{2}+\mu_{1} s^{3}+\frac{\mu_{2}}{2} \phi^{2} s+\mu_{2} v \phi s .
$$

The mass eigenstates can be parametrised in terms of a mixing angle $\theta$ as

$$
\begin{aligned}
& h_{1}=\phi \cos \theta-s \sin \theta, \\
& h_{2}=\phi \sin \theta+s \cos \theta,
\end{aligned}
$$

where $h_{1}$ is assumed to be the lighter Higgs boson with a mass of $125 \mathrm{GeV}$, and

$$
\tan 2 \theta=\frac{-\mu_{2} v}{\lambda v^{2}-\frac{1}{2} M^{2}}
$$

\footnotetext{
${ }^{6}$ Higgs singlet models with an additional $Z_{2}$ symmetry have generated some interest recently because of the possibility of the additional Higgs boson being a dark matter candidate, but here we consider the most general extension.
} 


\begin{tabular}{|l|cccc|}
\hline$M_{h_{2}}[\mathrm{GeV}]$ & 700 & 1000 & 1500 & 3000 \\
\hline$\theta_{1}$ & $\pi / 15$ & $\pi / 15$ & $\pi / 22$ & $\pi / 45$ \\
& $\approx 0.21$ & $\approx 0.21$ & $\approx 0.14$ & $\approx 0.07$ \\
\hline$\theta_{2}$ & $\pi / 8$ & $\pi / 8$ & $\pi / 12$ & $\pi / 24$ \\
& $\approx 0.39$ & $\approx 0.39$ & $\approx 0.26$ & $\approx 0.13$ \\
\hline
\end{tabular}

Table 1. Mixing angles $\theta_{1}$ and $\theta_{2}$ are given for all considered benchmark points, which are also characterised by $M_{h_{1}}=125 \mathrm{GeV}$ and $\mu_{1}=\lambda_{1}=\lambda_{2}=0$.

with

$$
-\frac{\pi}{4}<\theta<\frac{\pi}{4}
$$

under the condition $M^{2}>2 \lambda v^{2}$. The model has six independent parameters, which we choose to be $M_{h_{1}}, M_{h_{2}}, \theta, \mu_{1}, \lambda_{1}$ and $\lambda_{2}$. The dependent model parameters are:

$$
\begin{aligned}
\lambda & =\frac{\cos (2 \theta)\left(M_{h_{1}}^{2}-M_{h_{2}}^{2}\right)+M_{h_{1}}^{2}+M_{h_{2}}^{2}}{4 v^{2}}, \\
M^{2} & =\frac{M_{h_{2}}^{2}-M_{h_{1}}^{2}+\sec (2 \theta)\left(M_{h_{1}}^{2}+M_{h_{2}}^{2}\right)}{2 \sec (2 \theta)}, \\
\mu_{2} & =-\tan (2 \theta) \frac{\lambda v^{2}-\frac{1}{2} M^{2}}{v} .
\end{aligned}
$$

We set $M_{h_{1}}$ to $125 \mathrm{GeV}$ in accordance with the mass of the observed resonance and study four values for the mass of the heavy Higgs resonance: $M_{h_{2}}=700 \mathrm{GeV}, M_{h_{2}}=1 \mathrm{TeV}$, $M_{h_{2}}=1.5 \mathrm{TeV}$ and $M_{h_{2}}=3 \mathrm{TeV}$. We consider the mixing angles specified in table 1 . The lower values are consistent with current experimental limits [41, 42]. ${ }^{7}$ For each value of $M_{h_{2}}$, two angles, $\theta_{1}$ and $\theta_{2}$, have been chosen to illustrate how the studied interference effects change with the mixing angle. Furthermore, we consider model benchmark points with vanishing coupling parameters $\mu_{1}, \lambda_{1}$ and $\lambda_{2} . \quad\left(\lambda_{1}>0\right.$ is treated as approximately zero.) We emphasise that this does not imply that the $h_{2} \rightarrow h_{1} h_{1}$ and $h_{2} \rightarrow h_{1} h_{1} h_{1}$ decay widths are zero (if kinematically allowed). This is a consequence of the $\phi-s$ mixing. Inverting eqs. (2.5) and (2.6), one finds

$$
\begin{aligned}
& \phi=h_{1} \cos \theta+h_{2} \sin \theta, \\
& s=-h_{1} \sin \theta+h_{2} \cos \theta .
\end{aligned}
$$

Substituting eqs. (2.12) and (2.13) into the potential $V$, see eq. (2.4), more specifically into

$$
\frac{\lambda}{4} \phi^{4}, \lambda v \phi^{3}, \frac{\mu_{2}}{2} \phi^{2} s,
$$

\footnotetext{
${ }^{7}$ The perturbativity condition $\lambda<4 \pi$ imposes via eq. (2.9) the constraint $|\theta|<\theta_{0}$, which is satisfied for our benchmark points, since $\theta_{0} \geq 0.42$ for $M_{h_{1}}=125 \mathrm{GeV}$ and $200 \mathrm{GeV} \lesssim M_{h_{2}} \leq 3 \mathrm{TeV}$. We do not quantitatively consider the RG running of $\lambda$ to high scales, but note that the chosen mixing angles decrease with increasing $M_{h_{2}} \geq 1 \mathrm{TeV}$. Our $\theta$ values are compatible with the perturbativity constraints shown in refs. [41, 42].
} 
gives nonvanishing $h_{2} h_{1} h_{1} h_{1}$ and $h_{2} h_{1} h_{1}$ interactions, since $\lambda$ and $\mu_{2}$ are non-zero. ${ }^{8}$ The numerical values of $\Gamma\left(h_{2} \rightarrow h_{1} h_{1}\right), \Gamma\left(h_{2} \rightarrow h_{1} h_{1} h_{1}\right)$ and $\Gamma\left(h_{2} \rightarrow h_{1} h_{1} h_{1} h_{1}\right)$ for our benchmark points are given in appendix A.

\section{Calculational details}

We carry out calculations in the 1HSM (signal hypothesis) and the SM (null hypothesis). As input parameters, we adopt the recommendation of the LHC Higgs Cross Section Working Group in chapter I.1 of ref. [72] with $M_{V}^{\text {pole }}$ and $\Gamma_{V}^{\text {pole }}$ as given by eq. (I.1.7). ${ }^{9}$ We employ the $G_{\mu}$ scheme, where

$$
\cos \theta_{W}=\frac{M_{W}}{M_{Z}}, \quad \alpha=\frac{\sqrt{2}}{\pi} G_{F} M_{W}^{2} \sin ^{2} \theta_{W} .
$$

More specifically, we use $G_{F}=1.1663787 \times 10^{-5} \mathrm{GeV}^{-2}, M_{W}=80.35797 \mathrm{GeV}, M_{Z}=$ $91.15348 \mathrm{GeV}, \Gamma_{W}=2.08430 \mathrm{GeV}, \Gamma_{Z}=2.49427 \mathrm{GeV}, m_{t}=173.2 \mathrm{GeV}, \Gamma_{t}=1.369 \mathrm{GeV}$ and $m_{b}=4.92 \mathrm{GeV}$. Via eq. (3.1), we have $1 / \alpha \approx 132.36$ and $\sin ^{2} \theta_{W} \approx 0.222838$.

The PDF set PDF4LHC15_nlo_mc [73] with default $\alpha_{s}$ is used, and the CKM matrix is approximated by the identity matrix. ${ }^{10}$ The renormalisation and factorisation scales are set to $M_{W W} / 2$ for $W W$ production and $M_{t \bar{t}} / 2$ for $t \bar{t}$ production. The $p p$ collision energy is $\sqrt{s}=13 \mathrm{TeV}$. Finite top and bottom quark mass effects are fully taken into account. Lepton masses are neglected. As unstable particle states arise in the considered processes, the prescription of the complex-mass scheme $[75,76]$ is applied to all scattering amplitudes.

SM Higgs widths have been calculated using HDECAY [77, 78] and Prophecy4F [79-81]. For the SM Higgs with $M_{H}=125 \mathrm{GeV}$, one obtains $\Gamma_{H}=4.087 \times 10^{-3} \mathrm{GeV}$. The Higgs boson widths in the $1 \mathrm{HSM}$ are calculated as follows:

$$
\begin{aligned}
\Gamma_{h_{1}} & =\cos ^{2} \theta \Gamma_{H}\left(M_{h_{1}}\right), \\
\Gamma_{h_{2}} & =\sin ^{2} \theta \Gamma_{H}\left(M_{h_{2}}\right)+\Gamma\left(h_{2} \rightarrow n \times h_{1}\right),
\end{aligned}
$$

where $\Gamma_{H}(M)$ denotes the width of a SM Higgs boson with mass $M .^{11}$ We take into account decay modes, where $h_{2}$ decays into up to four $h_{1}$ bosons, i.e. $2 \leq n \leq 4$ in eq. (3.3). A custom implementation of the 1HSM in FEYNRules [82, 83] and the UFO [84] interface with MADGrAPH5_AMC@NLO [85] was used to calculate $\Gamma\left(h_{2} \rightarrow h_{1} h_{1}\right), \Gamma\left(h_{2} \rightarrow\right.$ $\left.h_{1} h_{1} h_{1}\right)$ and $\Gamma\left(h_{2} \rightarrow h_{1} h_{1} h_{1} h_{1}\right)$. The resulting partial decay widths are given in table 8 in appendix A. The results displayed in table 8 demonstrate that the $h_{2}$ width contributions from higher $h_{1}$ multiplicities are suppressed for all considered benchmark points. The resulting values for $\Gamma_{h_{1}}$ and $\Gamma_{h_{2}}$ and the corresponding $\Gamma / M$ ratios are given in table 2 .

\footnotetext{
${ }^{8}$ The corresponding Feynman rules are given in eqs. (331) and (334) in ref. [71].

${ }^{9}$ For $m_{t}$, we use the world average of $m_{t}^{\mathrm{OS}}$.

${ }^{10}$ In this context, the associated error is smaller than $0.01 \%[74]$.

${ }^{11}$ For $M=3 \mathrm{TeV}, \Gamma_{H}(M)$ cannot be obtained using HDECAY due to numerical problems for decay modes with $b$ quark loops caused by the tiny value of the running $b$ quark mass. For $M=3 \mathrm{TeV}$, we therefore approximate $\Gamma_{H}(M) \approx \Gamma(H \rightarrow W W)+\Gamma(H \rightarrow Z Z)$.
} 


\begin{tabular}{|c|c|cccc|}
\hline$\theta$ & $M_{h_{2}}[\mathrm{GeV}]$ & 700 & 1000 & 1500 & 3000 \\
\hline \multirow{4}{*}{$\theta_{1}$} & $\Gamma_{h_{1}}[\mathrm{GeV}]$ & $3.910(5) \times 10^{-3}$ & $3.910(5) \times 10^{-3}$ & $4.004(5) \times 10^{-3}$ & $4.067(5) \times 10^{-3}$ \\
& $\Gamma_{h_{1}} / M_{h_{1}}$ & $3.1283(4) \times 10^{-5}$ & $3.1283(4) \times 10^{-5}$ & $3.2034(4) \times 10^{-5}$ & $3.2537(4) \times 10^{-5}$ \\
\cline { 2 - 6 } & $\Gamma_{h_{2}}[\mathrm{GeV}]$ & $10.780(3)$ & $34.295(3)$ & $79.52(2)$ & $86.70(3)$ \\
& $\Gamma_{h_{2}} / M_{h_{2}}$ & $0.015400(4)$ & $0.034295(3)$ & $0.053013(7)$ & $0.028902(9)$ \\
\hline \multirow{6}{*}{$\theta_{2}$} & $\Gamma_{h_{1}}[\mathrm{GeV}]$ & $3.488(5) \times 10^{-3}$ & $3.488(5) \times 10^{-3}$ & $3.813(5) \times 10^{-3}$ & $4.017(5) \times 10^{-3}$ \\
& $\Gamma_{h_{1}} / M_{h_{1}}$ & $2.7908(4) \times 10^{-5}$ & $2.7908(4) \times 10^{-5}$ & $3.0506(4) \times 10^{-5}$ & $3.2139(4) \times 10^{-5}$ \\
\cline { 2 - 6 } & $\Gamma_{h_{2}}[\mathrm{GeV}]$ & $33.903(8)$ & $116.37(4)$ & $273.6(2)$ & $322.5(2)$ \\
& $\Gamma_{h_{2}} / M_{h_{2}}$ & $0.04843(2)$ & $0.11637(4)$ & $0.18240(8)$ & $0.10751(5)$ \\
\hline
\end{tabular}

Table 2. Decay widths and $\Gamma / M$ ratios of the two physical Higgs bosons $h_{1}$ and $h_{2}$ in the 1-HiggsSinglet extension of the SM for the considered benchmark points. Details as in table 1. The error due to rounding and numerical integration is given in brackets.
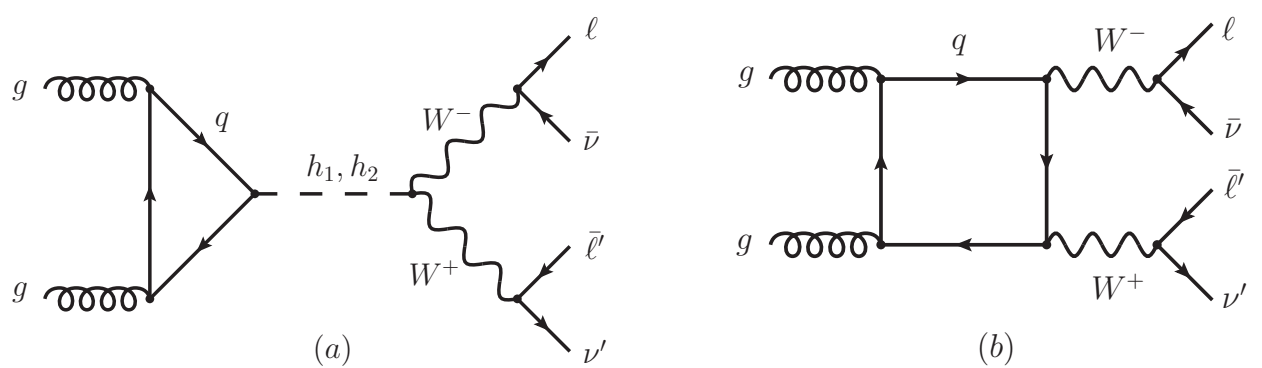

(b)

Figure 2. Representative Feynman graphs for $g g\left(\rightarrow\left\{h_{1}, h_{2}\right\}\right) \rightarrow W W \rightarrow 4$ leptons in the SM extended with a real scalar singlet field. The light $\left(h_{1}\right)$ and heavy Higgs $\left(h_{2}\right)$ production graphs (a) interfere with each other and the gluon-induced continuum background graphs (b).

We study Higgs boson production in gluon fusion at the LHC for the $W W$ and $t \bar{t}$ decay modes with subsequent fully-leptonic $W$ boson decays in the 1HSM:

$$
\begin{aligned}
& g g\left(\rightarrow\left\{h_{1}, h_{2}\right\}\right) \rightarrow W^{-} W^{+} \rightarrow \ell \bar{\nu} \bar{\ell}^{\prime} \nu^{\prime} \\
& g g\left(\rightarrow\left\{h_{1}, h_{2}\right\}\right) \rightarrow t \bar{t} \rightarrow b \bar{b} \ell \bar{\nu} \bar{\ell}^{\prime} \nu^{\prime} .
\end{aligned}
$$

The results presented in section 4 have been calculated at LO unless otherwise noted and are given for a single combination of different lepton flavours, for instance $\ell=e^{-}, \ell^{\prime}=\mu^{-}$.

Representative Feynman graphs for the light and heavy Higgs and interfering continuum background processes in the 1HSM are shown in figures 2 and 3. All considered amplitudes are at the one-loop level, except for the $g g \rightarrow t \bar{t}$ continuum background at LO. For the $t \bar{t}$ process shown in figure 3 , we note that continuum background graphs with $s$-channel gluon propagator do not interfere with the Higgs graphs, which have a colour singlet initial and final state. For the depicted continuum background graphs, only the colour singlet configurations - occurring with probability $1 /\left(N_{c}^{2}-1\right)$ - contribute to the signal-background interference. The corresponding SM graphs are obtained by substituting $h_{1}$ with $H$ and discarding $h_{2}$ contributions. The amplitudes are calculated using customised OpenLoops $[86,87]$ code, which is interfaced to the Sherpa Monte Carlo 


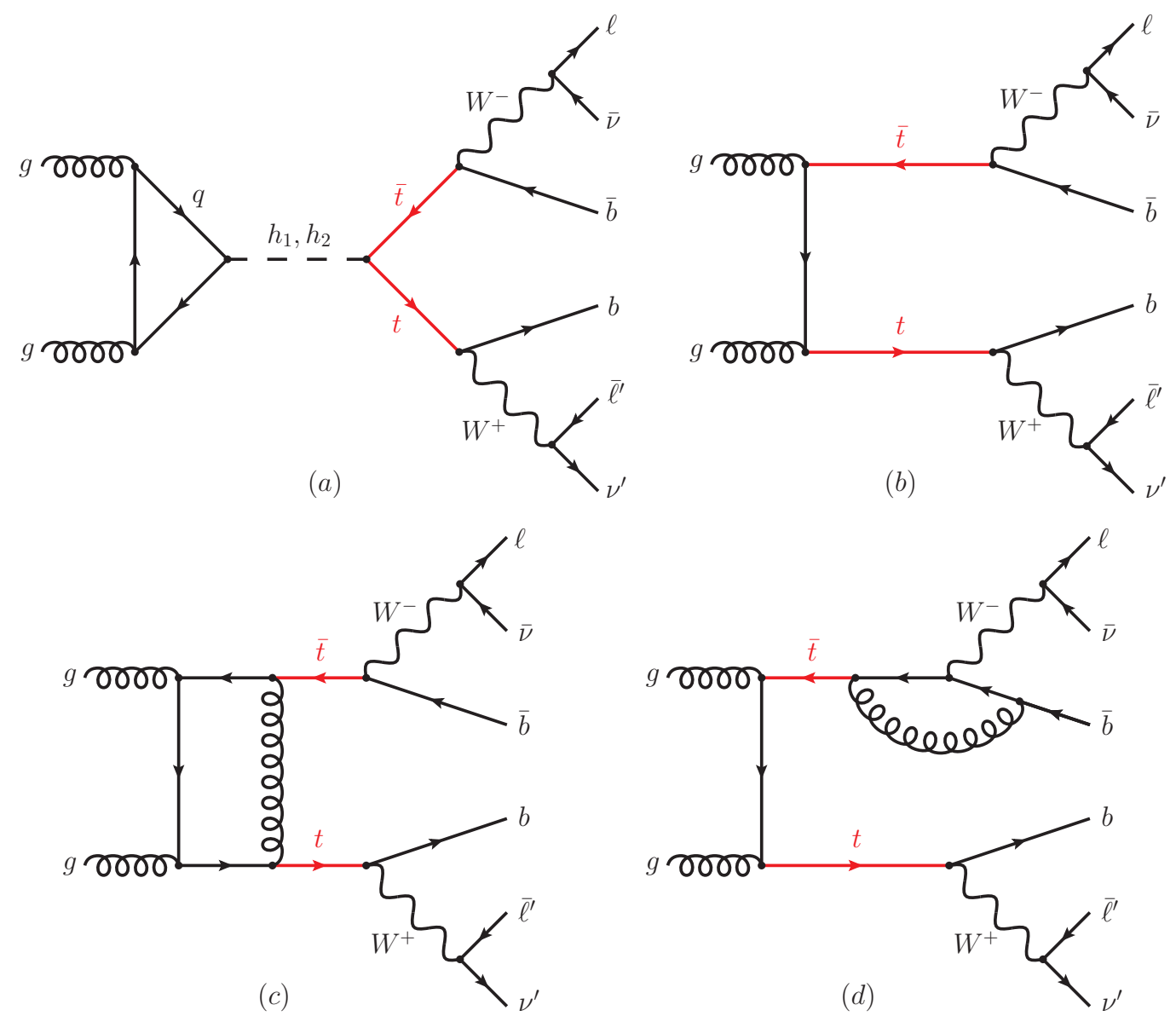

Figure 3. Representative Feynman graphs for $g g\left(\rightarrow\left\{h_{1}, h_{2}\right\}\right) \rightarrow t \bar{t} \rightarrow b \bar{b}+4$ leptons in the SM extended with a real scalar singlet field. The light $\left(h_{1}\right)$ and heavy Higgs $\left(h_{2}\right)$ production graphs (a) interfere with each other and the gluon-induced LO continuum background graphs (b). Interference with the gluon-induced continuum background at the one-loop level, for which representative graphs are shown in (c) and (d), is also considered. The process is calculated in double pole approximation with a pair of on-shell top quarks (red).

(MC) event generator [88, 89] and LHAPDF [90]. Since SHERPA-2.2.5 does not automatically generate phase space integrators for loop-induced processes, a customised approach is used. Full spin correlations are taken into account for all considered processes. For the top pair process, eq. (3.5), since our study focuses on the region with $2 m_{t}<M_{t \bar{t}}<M_{h_{2}}$, the double pole approximation [91] with a pair of on-shell top quark states - shown in red in figure 3 - is applied to simplify our calculations. It has been shown that higher-order corrections to interference can be larger than the interference at LO [66, 67]. In the case where LO involves tree-level amplitudes, this can be understood as follows: the relative phase that induces large interference arises primarily through the absorptive part of loop graphs. We therefore also calculate the interference between the LO Higgs amplitude $H$ in the SM and $h_{1}$ as well as $h_{2}$ in the 1HSM, see figure figure 3(a) - and the inter- 
fering continuum background amplitude at the one-loop level, see figures 3(c) and 3(d). ${ }^{12}$ Since the top quark states are treated in narrow-width approximation (NWA), factorising production and decay, nonfactorisable corrections are neglected. ${ }^{13}$

The one-loop continuum background amplitudes are affected by ultraviolet (UV) and infrared (IR) singularities, which are treated with conventional dimensional regularisation. OpenLoops uses the on-shell scheme to renormalise all masses. For all sufficiently inclusive transition probabilities ("IR-safe" observables), the IR poles cancel when the virtual corrections, represented by figures 3(c) and 3(d), are combined with the real emission corrections [94, 95] and the collinear counterterms, which, taken together, constitute the full next-to-leading order (NLO) corrections to the continuum background subprocess in eq. (3.5). ${ }^{14}$ In our calculations, we do not take into account the real emission corrections to the LO continuum background amplitude, i.e. to figure 3(b), because they do not interfere with the LO signal amplitude, see figure 3(a). We note that they would have to be included in a full NLO calculation of the signal-background interference, together with the real emission corrections to the LO signal amplitude. ${ }^{15}$ A full NLO calculation of the signal-background interference is beyond the scope of this work.

We note that our 1HSM and SM implementation in SHERPA+OPENLoops is included in the arXiv submission as ancillary file sherpa_openloops_code.tar.bz2.

\section{Results}

To take into account the fiducial selection at the LHC, we employ a simplified version of the experimental leptonic cuts used in ref. [98] and standard jet selection criteria [99]. More precisely, we apply: ${ }^{16}$

$$
\begin{aligned}
& p_{T \ell_{1}}>22 \mathrm{GeV}, \quad p_{T \ell_{2}}>15 \mathrm{GeV}, \quad\left|\eta_{\ell}\right|<2.5, \quad M_{\ell \bar{\ell}^{\prime}}>10 \mathrm{GeV}, \quad p_{T}>20 \mathrm{GeV}, \\
& p_{T j}>30 \mathrm{GeV}, \quad\left|\eta_{j}\right|<4.7, \quad \Delta R_{j \ell}>0.4 .
\end{aligned}
$$

Integrated results for the SM and all considered 1HSM benchmark points (see table 1) are shown in tables 4-7. Mod-squared amplitude contributions are specified using the abbreviations defined in table 3. For reference, a nonredundant complete set of integrated results is given in appendix $\mathrm{B}$.

\footnotetext{
${ }^{12}$ The NLO contribution from interference of 2-loop virtual corrections to the loop-induced Higgs amplitude with the tree-level continuum background amplitude is not taken into account. We note that this contribution includes multiscale 2-loop diagrams of the non-factorisable type shown in the centre of figure 9 in ref. [62], for which results are not yet available. We believe this tree-2-loop contribution is small compared to the 1-loop-1-loop contribution we compute, because tree-1-loop interference was found to be small compared to 1-loop-1-loop interference in similar processes [67, 92], but the non-factorisable contribution may be enhanced due to the lifted colour singlet final state restriction.

${ }^{13}$ In the inclusive case, nonfactorisable corrections are suppressed by $\Gamma_{t} / m_{t}$, i.e. $\sim 1 \%$ [93].

${ }^{14} \mathrm{~A}$ description of the structure of NLO calculations can be found in ref. [96]. In OpEnLoops, the coefficient defined in eq. (2.6) of ref. [96] is chosen according to eq. (2.7) therein.

${ }^{15}$ At full NLO, also $g q$ and $q \bar{q}$ subprocesses [97], which are quark-PDF suppressed at the LHC, formally contribute to the signal-background and $h_{1}-h_{2}$ interference, as illustrated for $0 \rightarrow g q \bar{q} Z Z$ in figure 2 of ref. [92].

${ }^{16}$ The $b$ and $\bar{b}$ quark in the final state are not jet-clustered in our LO study.
} 
To explore the differential dependence, various distributions have been calculated. In addition to the Higgs invariant mass distribution $\left(M_{W W}, M_{t \bar{t}}\right)$, we have also studied the transverse mass distribution of the $W W$ system $\left(M_{T, W W}\right)$, the dilepton invariant mass $\left(M_{\ell \bar{\ell}^{\prime}}\right)$ and the angular observables $\Delta \eta_{\ell \overline{\ell^{\prime}}}, \Delta \phi_{\ell \bar{\ell}^{\prime}}$ and $\Delta R_{\ell \bar{\ell}^{\prime}} .{ }^{17}$

Differential cross section distributions in the 1HSM for $W W$ production and the benchmark point with $M_{h_{2}}=1500 \mathrm{GeV}$ and mixing angle $\theta_{1}$ are displayed in figures 4-9 and for $t \bar{t}$ production and the benchmark point with $M_{h_{2}}=700 \mathrm{GeV}$ and mixing angle $\theta_{1}$ in figures 10-15. For reference, differential distributions in the SM are displayed in appendix C.

For $W W$ and $t \bar{t}$ production, invariant mass distributions of the relative deviation $\delta=R-1$ of the Higgs cross section including its interference with the background in the $1 \mathrm{HSM}$ with $M_{h_{2}}=\{700,1000,1500,3000\} \mathrm{GeV}$ and mixing angles $\theta_{1}$ and $\theta_{2}$ compared to the SM are shown in figures 16-21. More specifically, $R$ is the ratio of $\sigma\left(h_{1+2}+\mathrm{I}(\mathrm{C})\right)$ to $\sigma(H+\mathrm{I}(\mathrm{C}))$ or for $t \bar{t}$ production also $\sigma\left(h_{1+2}+\mathrm{I}\left(\mathrm{C}_{+\circlearrowleft}\right)\right)$ to $\sigma\left(H+\mathrm{I}\left(\mathrm{C}_{+\circlearrowleft}\right)\right)$, i.e. including the virtual corrections to the continuum background.

Furthermore, $M_{t \bar{t}}, M_{\ell \bar{\ell}^{\prime}}, \Delta \eta_{\ell \bar{\ell}^{\prime}}$ and $\Delta \phi_{\ell \bar{\ell}^{\prime}}$ distributions of the relative deviation $\delta=R-1$ of the Higgs interference with the background without and with the virtual corrections $\left(\mathcal{M}_{\text {cont,loop }}\right)$ in the SM and $1 \mathrm{HSM}$ with $M_{h_{2}}=\{700,1000\} \mathrm{GeV}$ and mixing angles $\left\{\theta_{1}, \theta_{2}\right\}$ for $g g\left(\rightarrow\left\{h_{1}, h_{2}, H\right\}\right) \rightarrow t \bar{t} \rightarrow b \bar{b} \ell \bar{\nu} \bar{\ell}^{\prime} \nu^{\prime}$ are displayed in figures 22-25. Here, $R$ is the ratio of $\sigma\left(\mathrm{I}\left(H, \mathrm{C}_{+\circlearrowleft}\right)\right)$ to $\sigma(\mathrm{I}(H, \mathrm{C}))$ and $\sigma\left(\mathrm{I}\left(h_{1+2}, \mathrm{C}_{+} \circlearrowleft\right)\right)$ to $\sigma\left(\mathrm{I}\left(h_{1+2}, \mathrm{C}\right)\right)$ in the SM and 1HSM, respectively.

Supplementary figures with distributions for all studied quantities, models and benchmark points are available at this URL: http://users.hepforge.org/ nkauer/arXiv/plots_08May2019.pdf [100].

\footnotetext{
${ }^{17} M_{T, W W}$ is defined as in eq. (3.6) in ref. [52].
} 


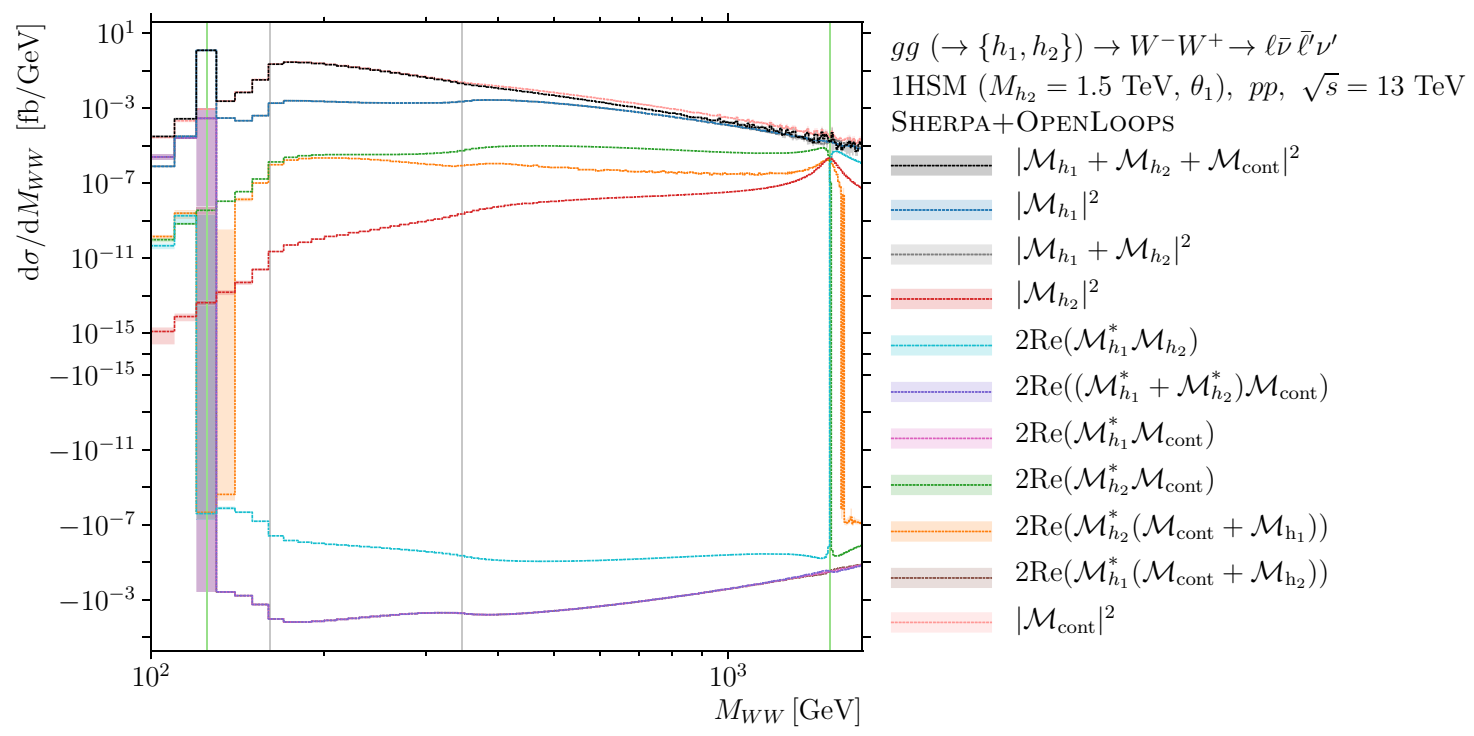

Figure 4. $M_{W W}$ distributions for the signal process $g g\left(\rightarrow\left\{h_{1}, h_{2}\right\}\right) \rightarrow W^{-} W^{+} \rightarrow \ell \bar{\nu} \bar{\ell}^{\prime} \nu^{\prime}$ in the 1-Higgs-Singlet Extension of the SM (1HSM) with $M_{h_{2}}=1500 \mathrm{GeV}$ and mixing angle $\theta_{1}$ (see table 1) including its interference with the background in $p p$ collisions at $\sqrt{s}=13 \mathrm{TeV}$. Vertical lines indicate the position of the $W W$ and $t \bar{t}$ thresholds (grey) and of the Higgs resonances (green). The selection cuts in (4.1) are applied. Cross sections are given for a single lepton flavour combination. The bands show the MC integration error estimate (90\% confidence interval).

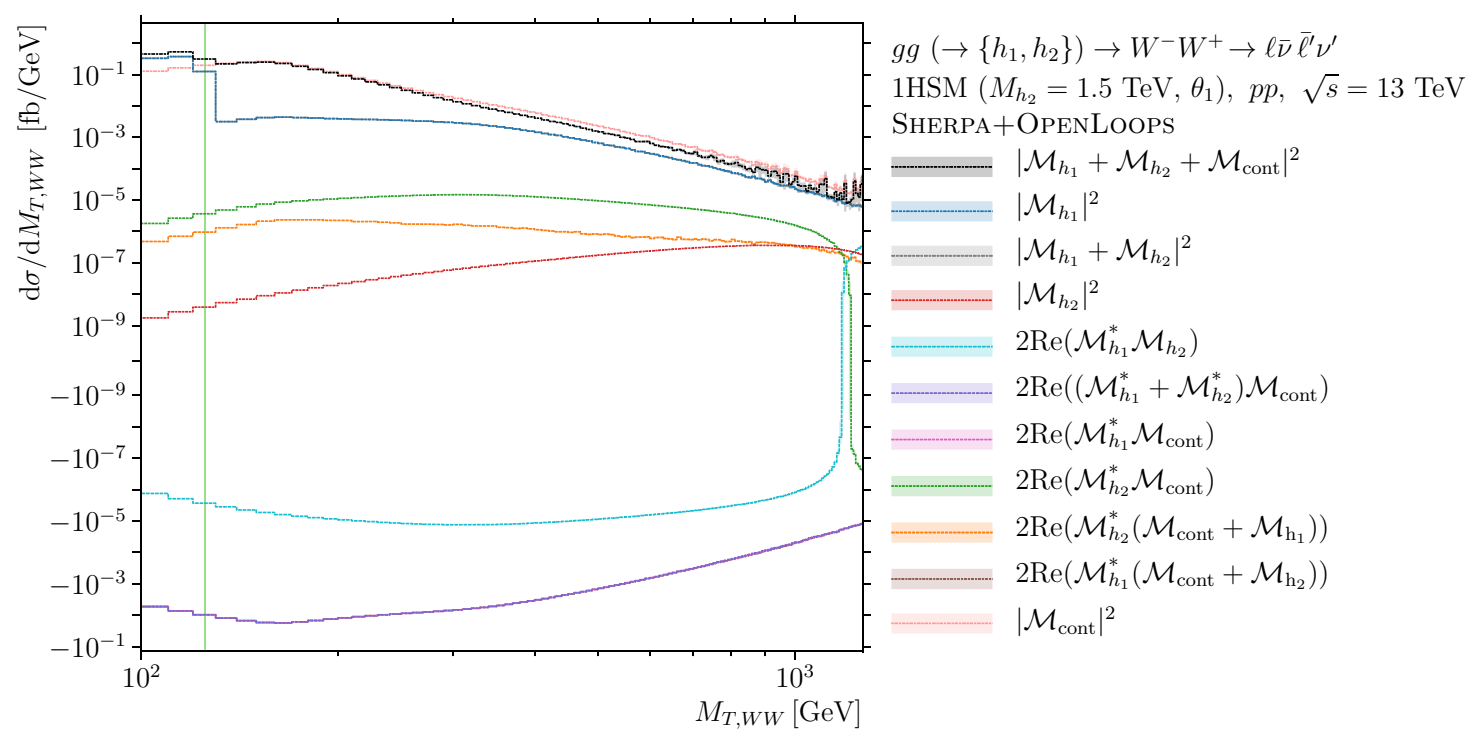

Figure 5. $\quad M_{T, W W}$ distributions for the signal process $g g\left(\rightarrow\left\{h_{1}, h_{2}\right\}\right) \rightarrow W^{-} W^{+} \rightarrow \ell \bar{\nu} \bar{\ell}^{\prime} \nu^{\prime}$ in the 1HSM $\left(M_{h_{2}}=1500 \mathrm{GeV}, \theta_{1}\right)$ including its interference with the background in $p p$ collisions at $\sqrt{s}=13 \mathrm{TeV}$. Other details as in figure 4 . 


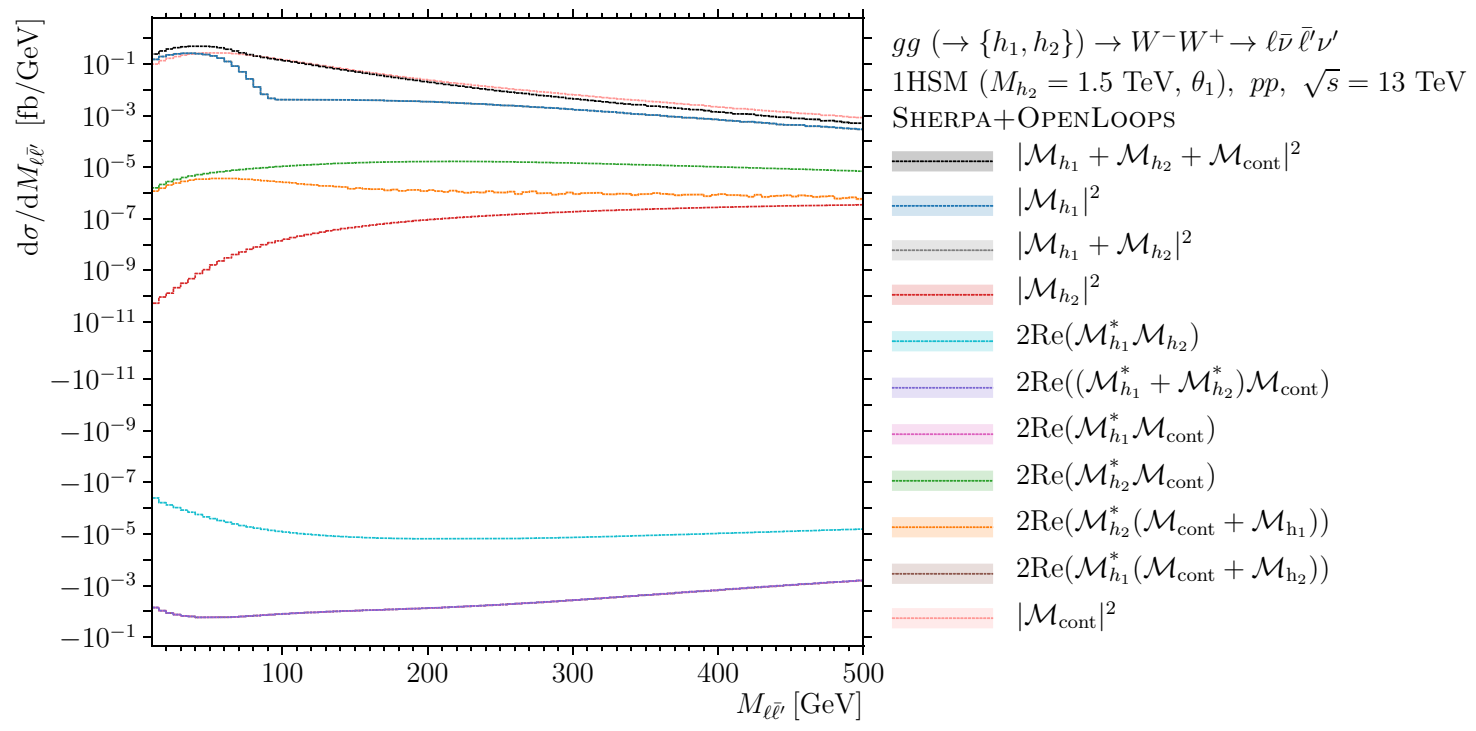

Figure 6. $M_{\ell \bar{\ell}^{\prime}}$ distributions for the signal process $g g\left(\rightarrow\left\{h_{1}, h_{2}\right\}\right) \rightarrow W^{-} W^{+} \rightarrow \ell \bar{\nu} \bar{\ell}^{\prime} \nu^{\prime}$ in the $1 \mathrm{HSM}\left(M_{h_{2}}=1500 \mathrm{GeV}, \theta_{1}\right)$ including its interference with the background in $p p$ collisions at $\sqrt{s}=13 \mathrm{TeV}$. Other details as in figure 4 .

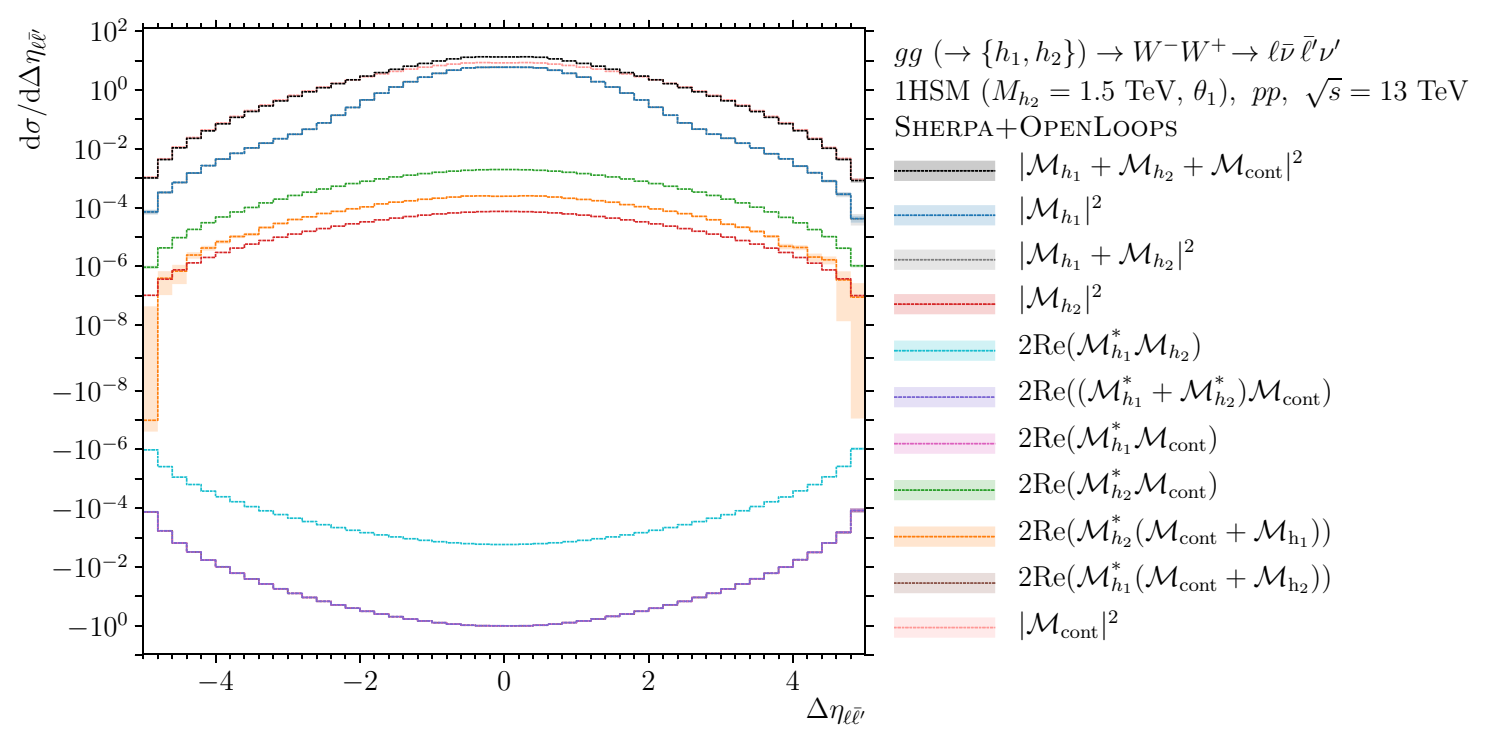

Figure 7. $\Delta \eta_{\ell \bar{\ell}^{\prime}}$ distributions for the signal process $g g\left(\rightarrow\left\{h_{1}, h_{2}\right\}\right) \rightarrow W^{-} W^{+} \rightarrow \ell \bar{\nu} \bar{\ell}^{\prime} \nu^{\prime}$ in the 1HSM $\left(M_{h_{2}}=1500 \mathrm{GeV}, \theta_{1}\right)$ including its interference with the background in $p p$ collisions at $\sqrt{s}=13 \mathrm{TeV}$. Other details as in figure 4 . 


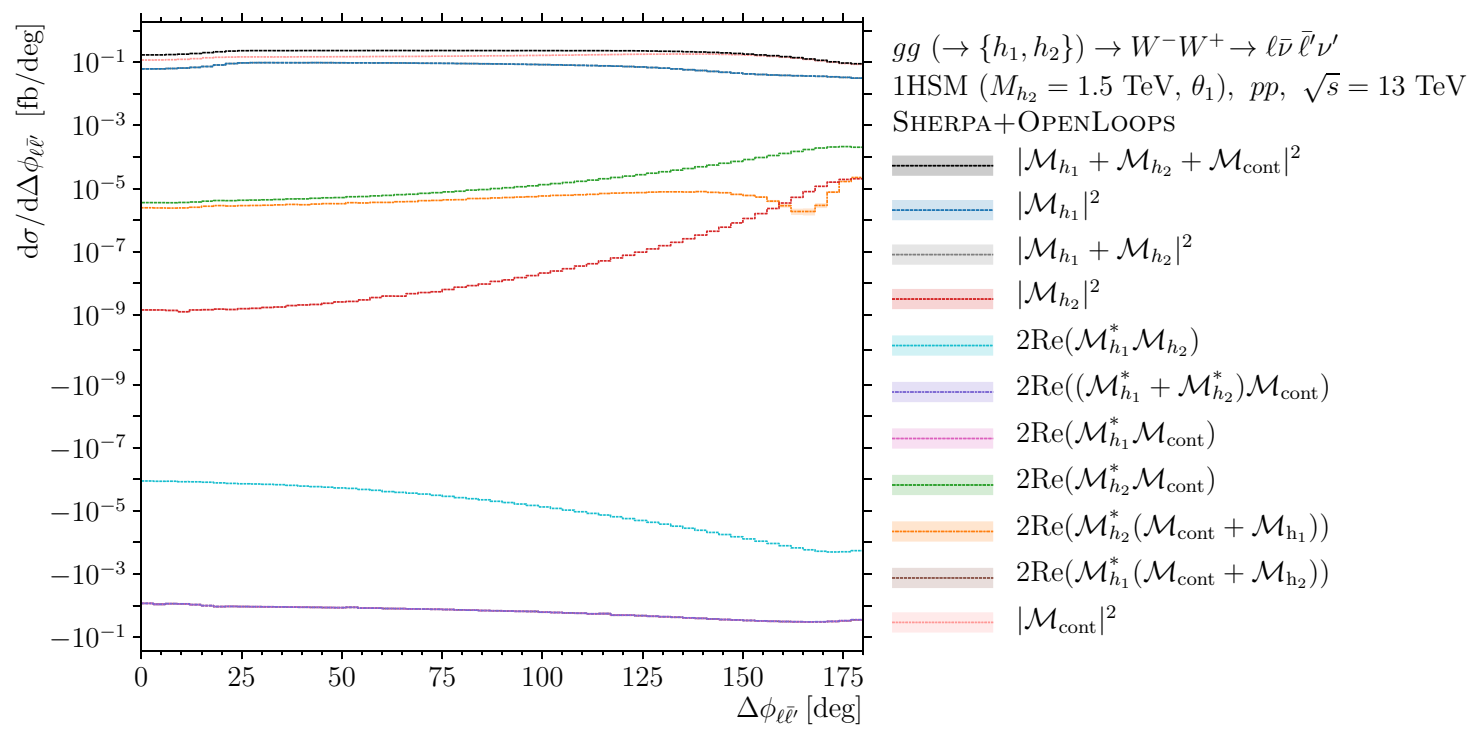

Figure 8. $\Delta \phi_{\ell \bar{\ell}^{\prime}}$ distributions for the signal process $g g\left(\rightarrow\left\{h_{1}, h_{2}\right\}\right) \rightarrow W^{-} W^{+} \rightarrow \ell \bar{\nu} \bar{\ell}^{\prime} \nu^{\prime}$ in the 1HSM $\left(M_{h_{2}}=1500 \mathrm{GeV}, \theta_{1}\right)$ including its interference with the background in $p p$ collisions at $\sqrt{s}=13 \mathrm{TeV}$. Other details as in figure 4 .

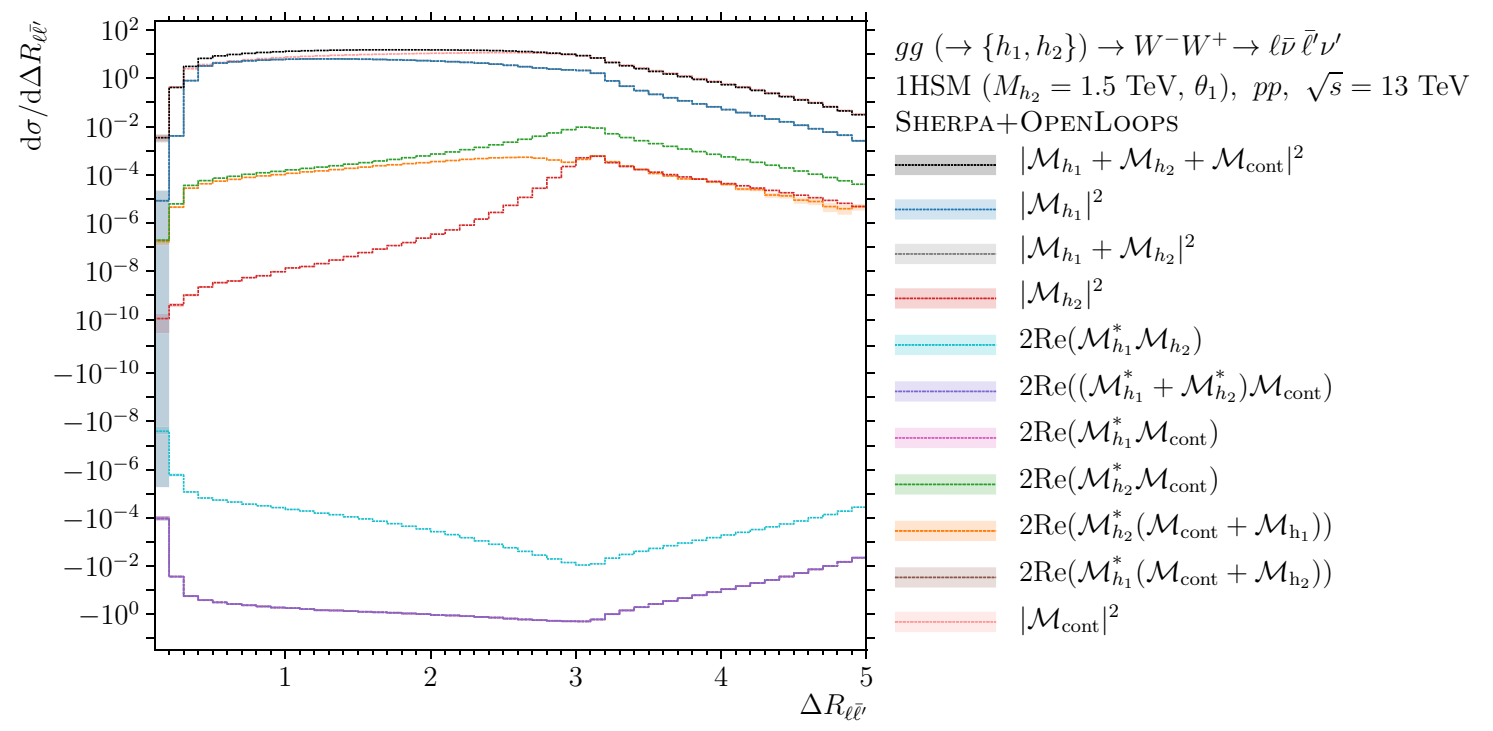

Figure 9. $\Delta R_{\ell \bar{\ell}^{\prime}}$ distributions for the signal process $g g\left(\rightarrow\left\{h_{1}, h_{2}\right\}\right) \rightarrow W^{-} W^{+} \rightarrow \ell \bar{\nu} \bar{\ell}^{\prime} \nu^{\prime}$ in the 1HSM $\left(M_{h_{2}}=1500 \mathrm{GeV}, \theta_{1}\right)$ including its interference with the background in $p p$ collisions at $\sqrt{s}=13 \mathrm{TeV}$. Other details as in figure 4 . 


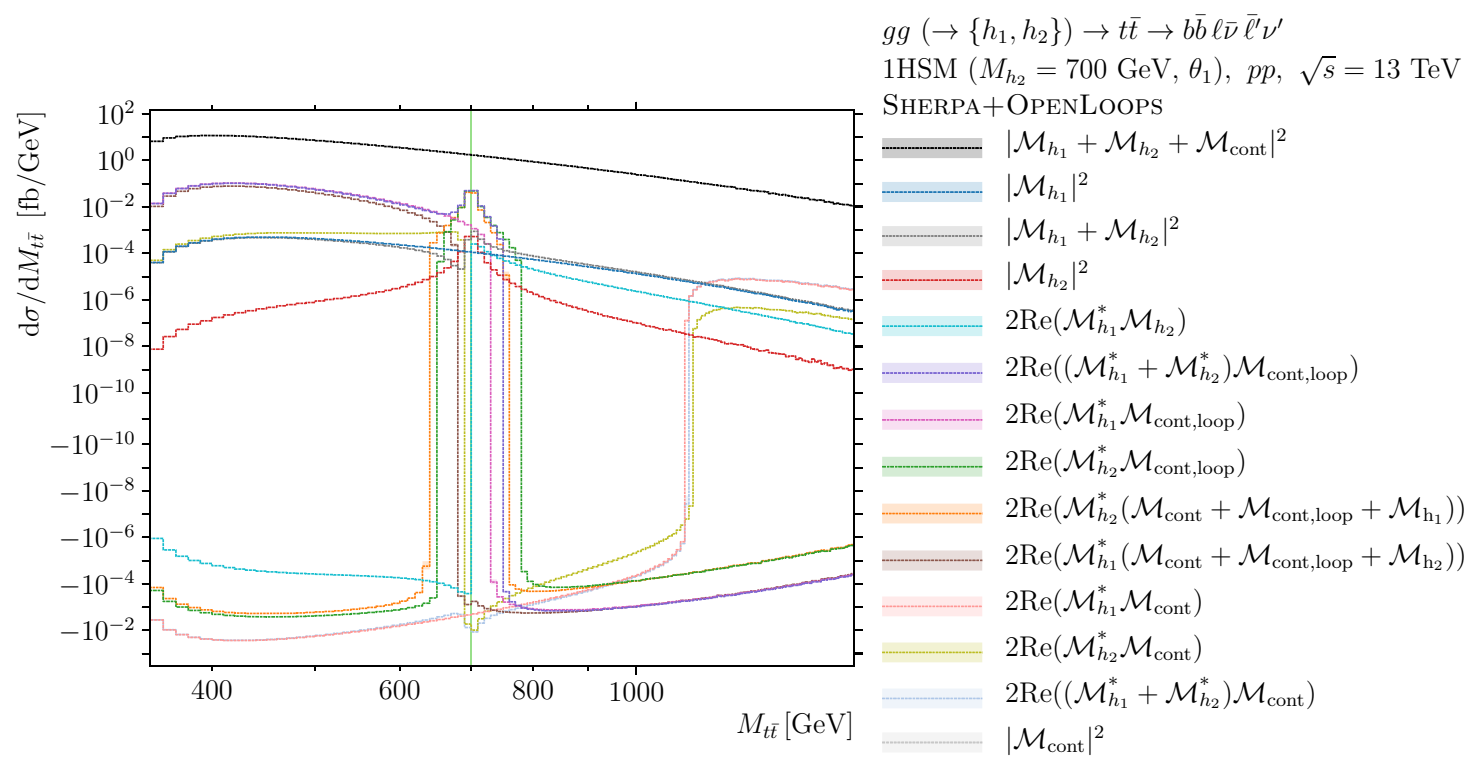

Figure 10. $M_{t \bar{t}}$ distributions for the signal process $g g\left(\rightarrow\left\{h_{1}, h_{2}\right\}\right) \rightarrow t \bar{t} \rightarrow b \bar{b} \ell \bar{\nu} \bar{\ell}^{\prime} \nu^{\prime}$ in the 1HSM $\left(M_{h_{2}}=700 \mathrm{GeV}, \theta_{1}\right)$ including its interference with the background in $p p$ collisions at $\sqrt{s}=13 \mathrm{TeV}$. Other details as in figure 4 .

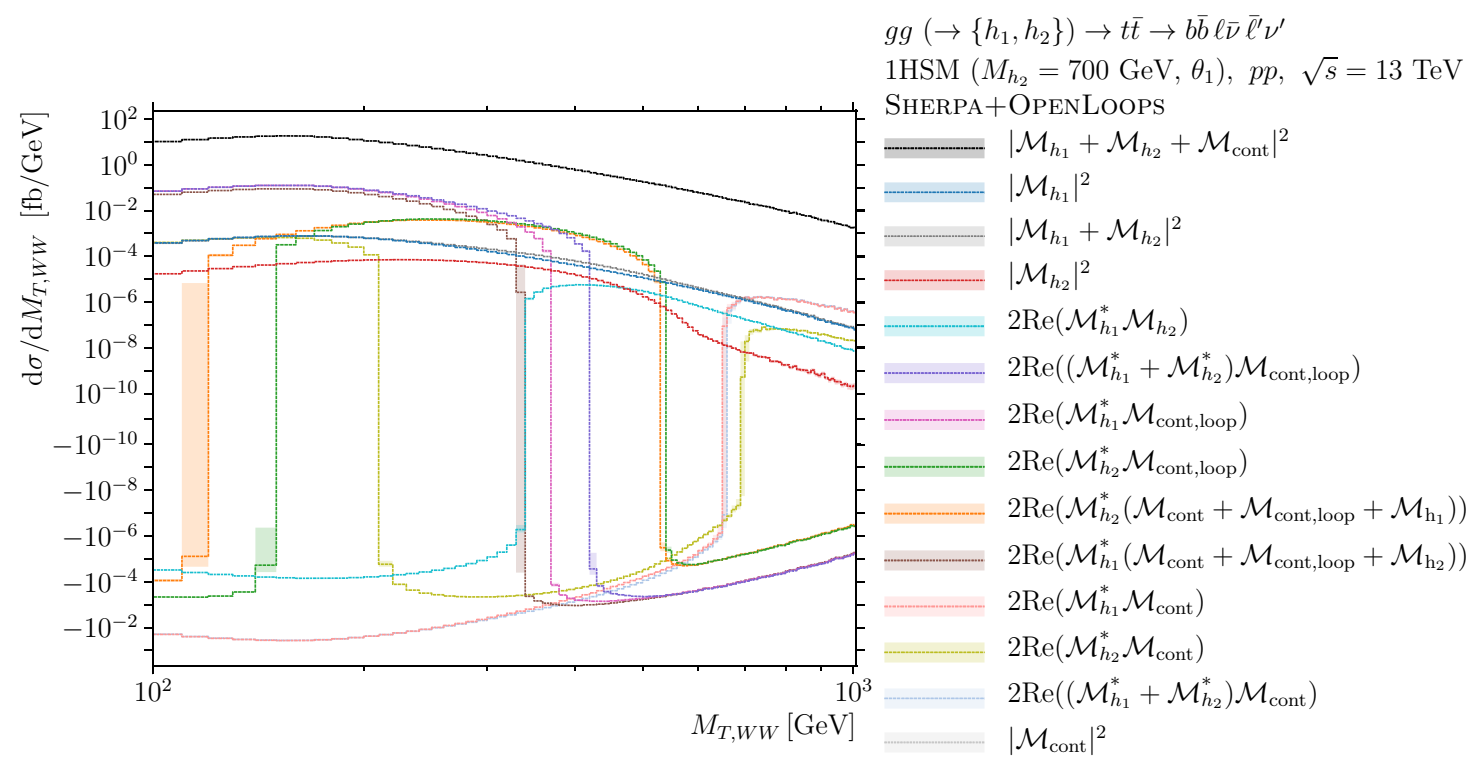

Figure 11. $M_{T, W W}$ distributions for the signal process $g g\left(\rightarrow\left\{h_{1}, h_{2}\right\}\right) \rightarrow t \bar{t} \rightarrow b \bar{b} \ell \bar{\nu} \bar{\ell}^{\prime} \nu^{\prime}$ in the $1 \mathrm{HSM}\left(M_{h_{2}}=700 \mathrm{GeV}, \theta_{1}\right)$ including its interference with the background in $p p$ collisions at $\sqrt{s}=13 \mathrm{TeV}$. Other details as in figure 4 . 


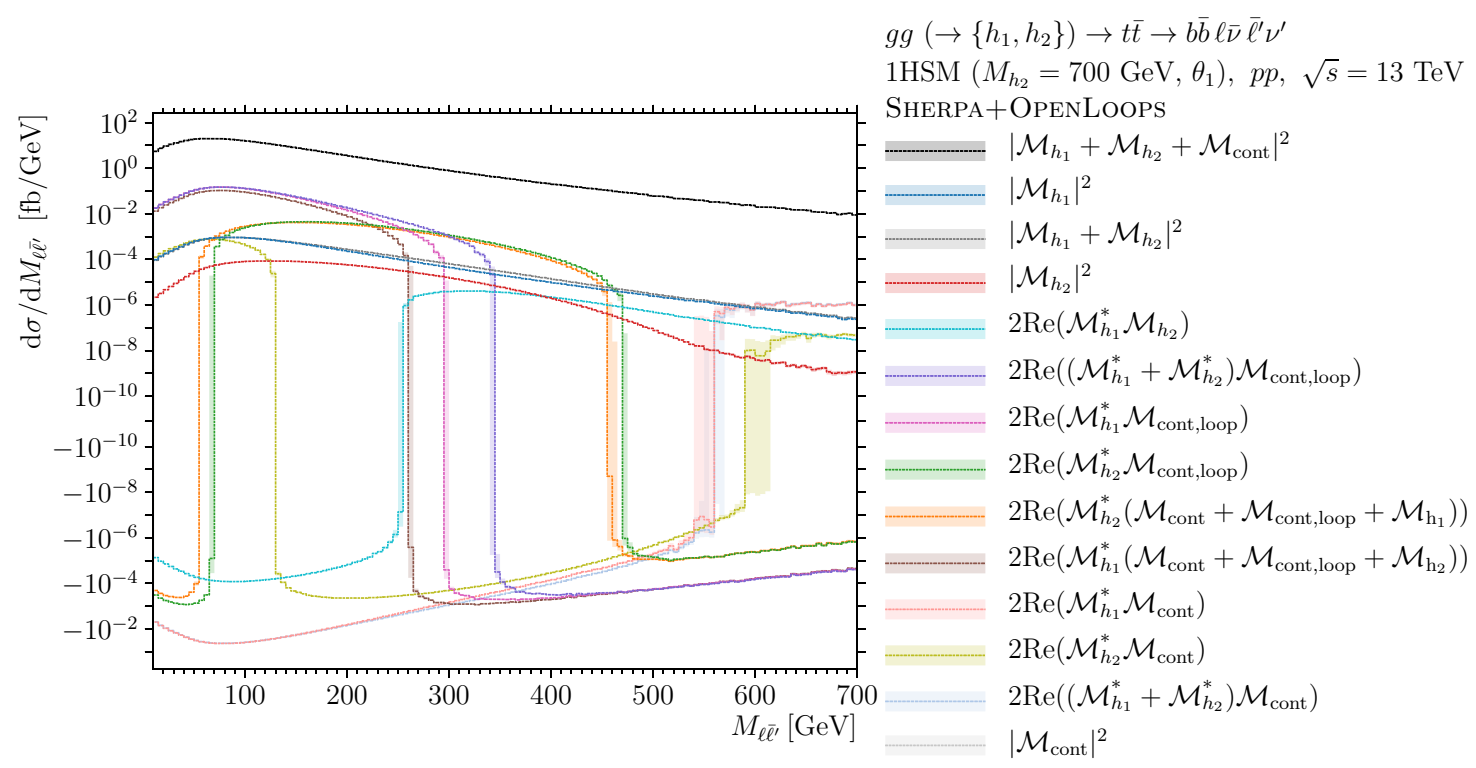

Figure 12. $M_{\ell \bar{\ell}^{\prime}}$ distributions for the signal process $g g\left(\rightarrow\left\{h_{1}, h_{2}\right\}\right) \rightarrow t \bar{t} \rightarrow b \bar{b} \ell \bar{\nu} \bar{\ell}^{\prime} \nu^{\prime}$ in the 1HSM $\left(M_{h_{2}}=700 \mathrm{GeV}, \theta_{1}\right)$ including its interference with the background in $p p$ collisions at $\sqrt{s}=13 \mathrm{TeV}$. Other details as in figure 10.

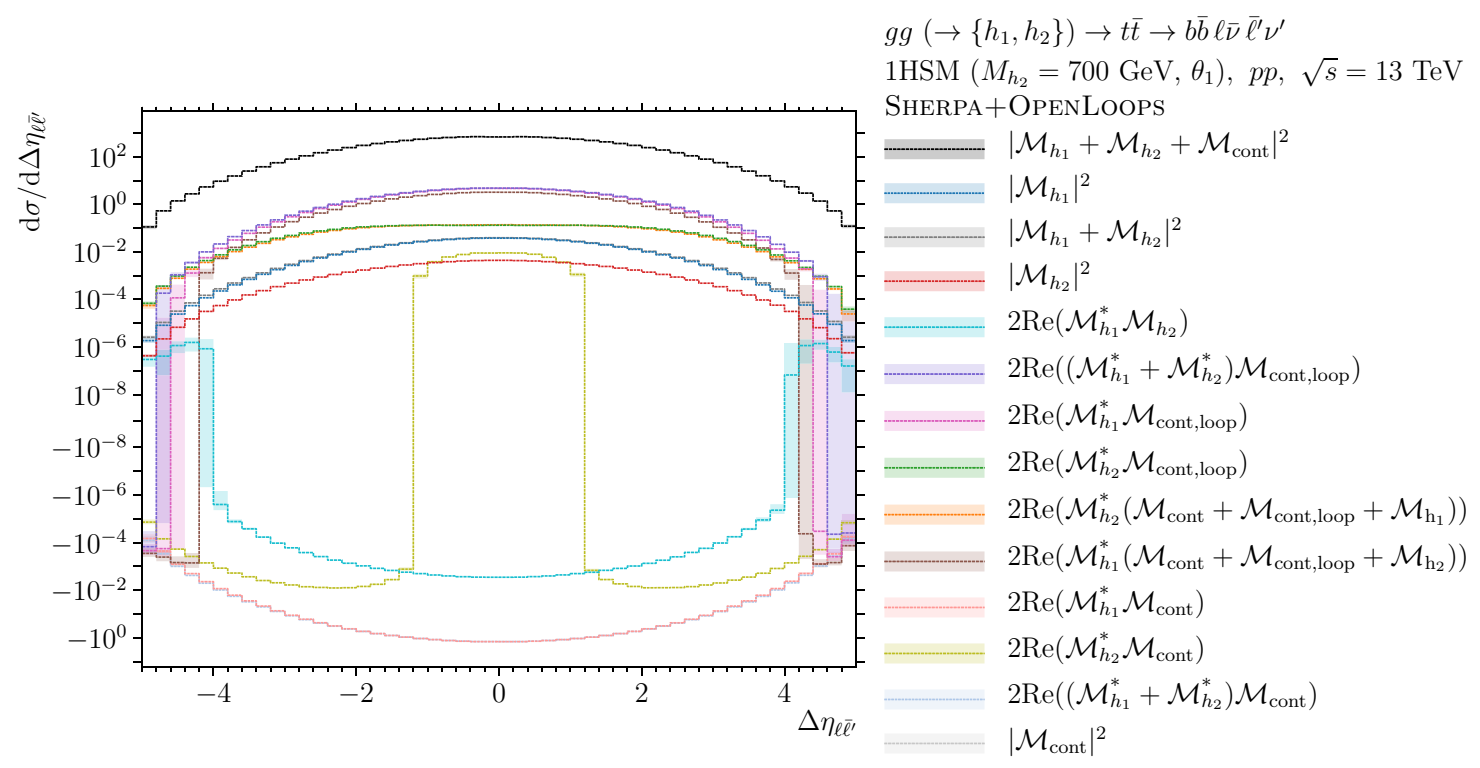

Figure 13. $\Delta \eta_{\ell \bar{\ell}^{\prime}}$ distributions for the signal process $g g\left(\rightarrow\left\{h_{1}, h_{2}\right\}\right) \rightarrow t \bar{t} \rightarrow b \bar{b} \ell \bar{\nu} \bar{\ell}^{\prime} \nu^{\prime}$ in the $1 \mathrm{HSM}\left(M_{h_{2}}=700 \mathrm{GeV}, \theta_{1}\right)$ including its interference with the background in $p p$ collisions at $\sqrt{s}=13 \mathrm{TeV}$. Other details as in figure 10 . 


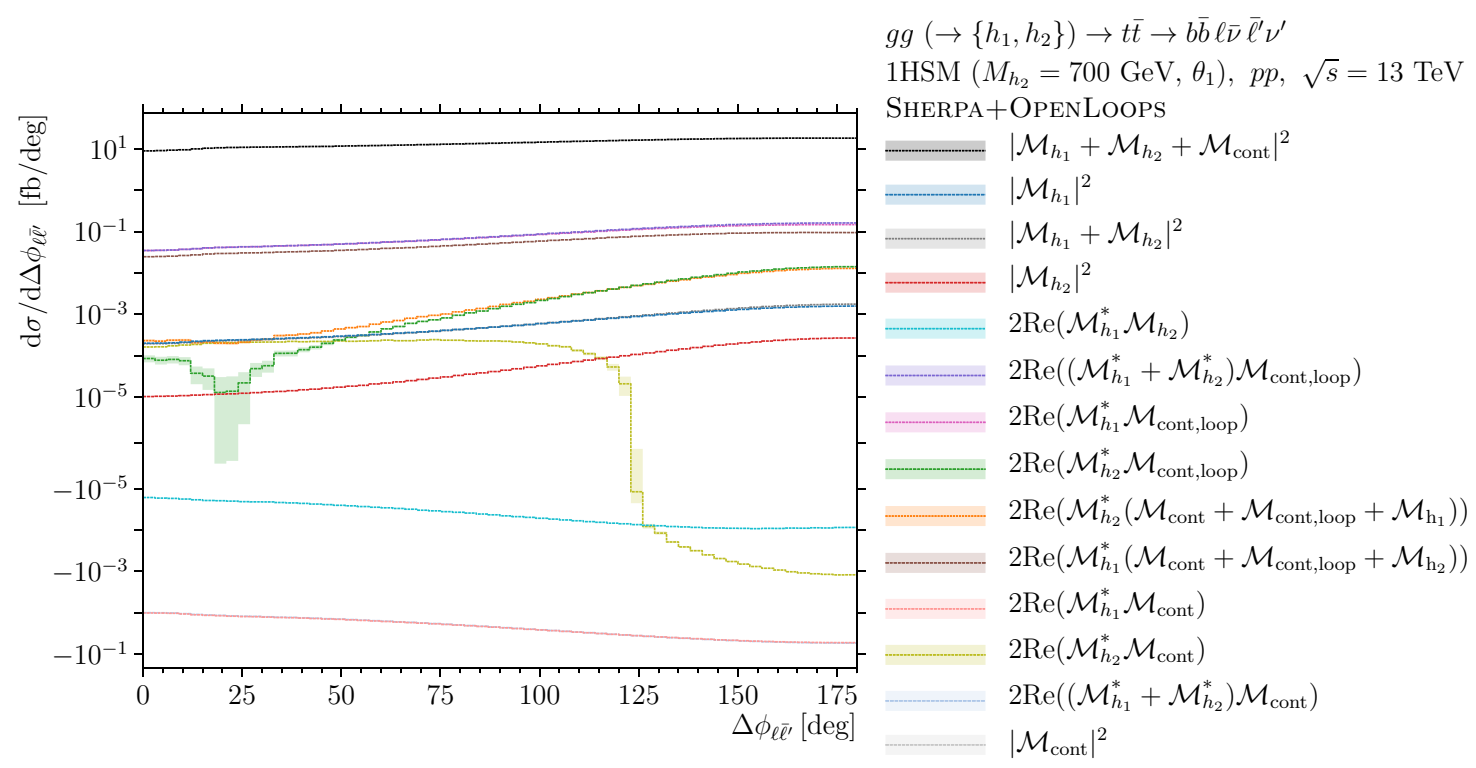

Figure 14. $\Delta \phi_{\ell \bar{\ell}^{\prime}}$ distributions for the signal process $g g\left(\rightarrow\left\{h_{1}, h_{2}\right\}\right) \rightarrow t \bar{t} \rightarrow b \bar{b} \ell \bar{\nu} \bar{\ell}^{\prime} \nu^{\prime}$ in the $1 \mathrm{HSM}\left(M_{h_{2}}=700 \mathrm{GeV}, \theta_{1}\right)$ including its interference with the background in $p p$ collisions at $\sqrt{s}=13 \mathrm{TeV}$. Other details as in figure 10 .

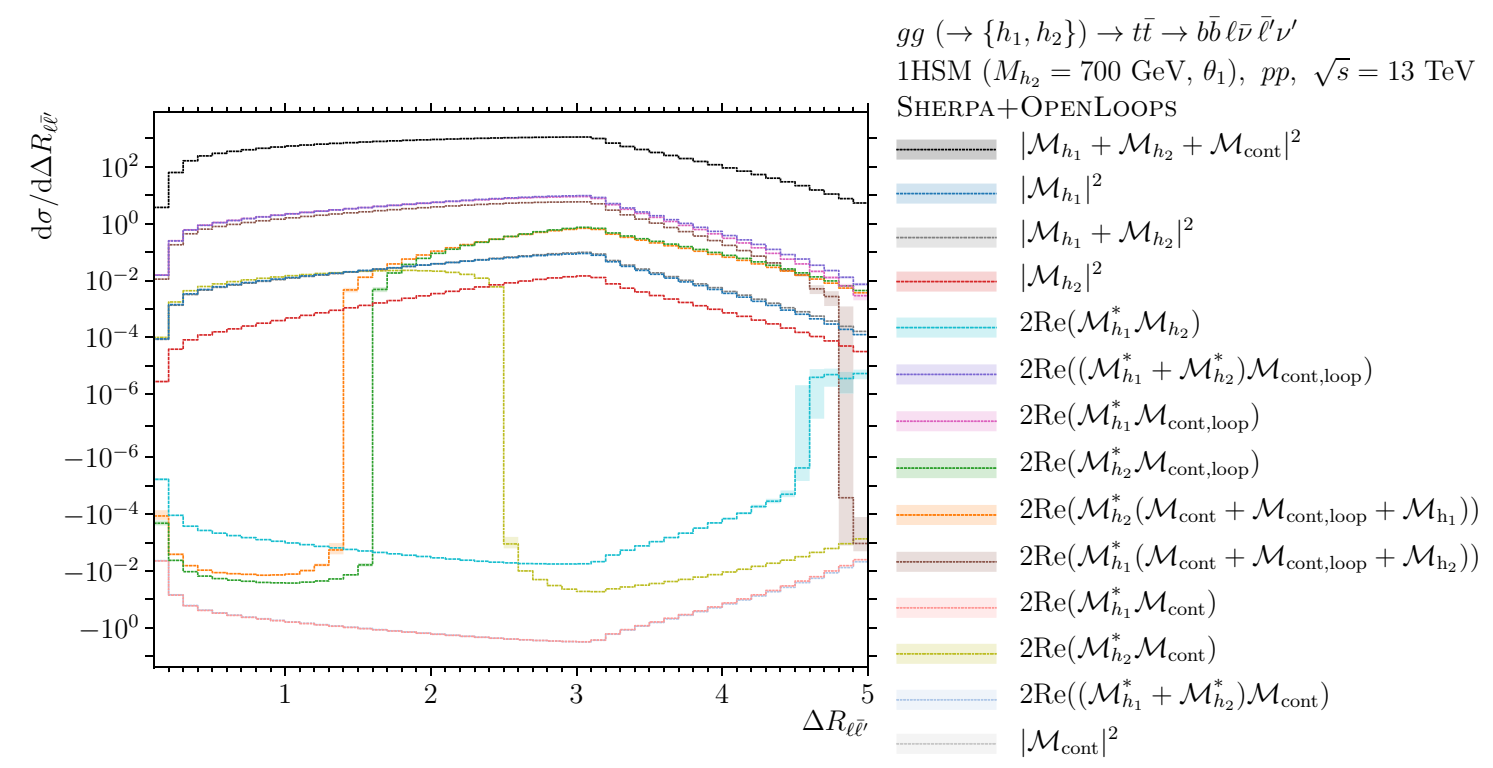

Figure 15. $\Delta R_{\ell \bar{\ell}^{\prime}}$ distributions for the signal process $g g\left(\rightarrow\left\{h_{1}, h_{2}\right\}\right) \rightarrow t \bar{t} \rightarrow b \bar{b} \ell \bar{\nu} \bar{\ell}^{\prime} \nu^{\prime}$ in the $1 \mathrm{HSM}\left(M_{h_{2}}=700 \mathrm{GeV}, \theta_{1}\right)$ including its interference with the background in $p p$ collisions at $\sqrt{s}=13 \mathrm{TeV}$. Other details as in figure 10 . 


\begin{tabular}{|c|c|}
\hline Label & $|\mathcal{M}|^{2}$ \\
\hline $\mathrm{Sq}(\mathrm{C})$ & $\left|\mathcal{M}_{\text {cont }}\right|^{2}$ \\
\hline $\operatorname{Sq}(H)$ & $\left|\mathcal{M}_{H}\right|^{2}$ \\
\hline $\mathrm{I}(H, \mathrm{C})$ & $2 \operatorname{Re}\left(\mathcal{M}_{H}^{*} \mathcal{M}_{\text {cont }}\right)$ \\
\hline$H+\mathrm{I}(\mathrm{C})$ & $\left|\mathcal{M}_{H}\right|^{2}+2 \operatorname{Re}\left(\mathcal{M}_{H}^{*} \mathcal{M}_{\text {cont }}\right)$ \\
\hline $\mathrm{I}\left(H, \mathrm{C}_{\circlearrowleft}\right)$ & $2 \operatorname{Re}\left(\mathcal{M}_{H}^{*} \mathcal{M}_{\text {cont,loop }}\right)$ \\
\hline $\mathrm{I}\left(H, \mathrm{C}_{+\circlearrowleft}\right)$ & $2 \operatorname{Re}\left(\mathcal{M}_{H}^{*}\left(\mathcal{M}_{\text {cont }}+\mathcal{M}_{\text {cont,loop }}\right)\right)$ \\
\hline$H+\mathrm{I}\left(\mathrm{C}_{+\circlearrowleft}\right)$ & $\left|\mathcal{M}_{H}\right|^{2}+2 \operatorname{Re}\left(\mathcal{M}_{H}^{*}\left(\mathcal{M}_{\text {cont }}+\mathcal{M}_{\text {cont,loop }}\right)\right)$ \\
\hline $\operatorname{Sq}\left(h_{1}\right)$ & $\left|\mathcal{M}_{h_{1}}\right|^{2}$ \\
\hline $\operatorname{Sq}\left(h_{2}\right)$ & $\left|\mathcal{M}_{h_{2}}\right|^{2}$ \\
\hline $\mathrm{I}\left(h_{1}, h_{2}\right)$ & $2 \operatorname{Re}\left(\mathcal{M}_{h_{1}}^{*} \mathcal{M}_{h_{2}}\right)$ \\
\hline$h_{2}+\mathrm{I}\left(h_{1}\right)$ & $\left|\mathcal{M}_{h_{2}}\right|^{2}+2 \operatorname{Re}\left(\mathcal{M}_{h_{1}}^{*} \mathcal{M}_{h_{2}}\right)$ \\
\hline $\mathrm{I}\left(h_{1}, \mathrm{C}\right)$ & $2 \operatorname{Re}\left(\mathcal{M}_{h_{1}}^{*} \mathcal{M}_{\text {cont }}\right)$ \\
\hline $\mathrm{I}\left(h_{2}, \mathrm{C}\right)$ & $2 \operatorname{Re}\left(\mathcal{M}_{h_{2}}^{*} \mathcal{M}_{\text {cont }}\right)$ \\
\hline $\operatorname{Sq}\left(h_{1+2}\right)$ & $\left|\mathcal{M}_{h_{1}}+\mathcal{M}_{h_{2}}\right|^{2}$ \\
\hline $\mathrm{I}\left(h_{1+2}, \mathrm{C}\right)$ & $2 \operatorname{Re}\left(\left(\mathcal{M}_{h_{1}}^{*}+\mathcal{M}_{h_{2}}^{*}\right) \mathcal{M}_{\text {cont }}\right)$ \\
\hline$h_{1+2}+\mathrm{I}(\mathrm{C})$ & $\left|\mathcal{M}_{h_{1}}+\mathcal{M}_{h_{2}}\right|^{2}+2 \operatorname{Re}\left(\left(\mathcal{M}_{h_{1}}^{*}+\mathcal{M}_{h_{2}}^{*}\right) \mathcal{M}_{\text {cont }}\right)$ \\
\hline $\mathrm{I}\left(h_{2}, \mathrm{C}+h_{1}\right)$ & $2 \operatorname{Re}\left(\mathcal{M}_{h_{2}}^{*}\left(\mathcal{M}_{\text {cont }}+\mathcal{M}_{\mathrm{h}_{1}}\right)\right)$ \\
\hline$h_{2}+\mathrm{I}\left(\mathrm{C}+h_{1}\right)$ & $\left|\mathcal{M}_{h_{2}}\right|^{2}+2 \operatorname{Re}\left(\mathcal{M}_{h_{2}}^{*}\left(\mathcal{M}_{\text {cont }}+\mathcal{M}_{\mathrm{h}_{1}}\right)\right)$ \\
\hline $\mathrm{I}\left(h_{1}, \mathrm{C}+h_{2}\right)$ & $2 \operatorname{Re}\left(\mathcal{M}_{h_{1}}^{*}\left(\mathcal{M}_{\text {cont }}+\mathcal{M}_{\mathrm{h}_{2}}\right)\right)$ \\
\hline $\mathrm{I}\left(h_{1}, \mathrm{C}_{\circlearrowleft}\right)$ & $2 \operatorname{Re}\left(\mathcal{M}_{h_{1}}^{*} \mathcal{M}_{\text {cont,loop }}\right)$ \\
\hline $\mathrm{I}\left(h_{2}, \mathrm{C}_{\circlearrowleft}\right)$ & $2 \operatorname{Re}\left(\mathcal{M}_{h_{2}}^{*} \mathcal{M}_{\text {cont,loop }}\right)$ \\
\hline$h_{1+2}+\mathrm{I}\left(\mathrm{C}_{+} \circlearrowleft\right)$ & $\left|\mathcal{M}_{h_{1}}+\mathcal{M}_{h_{2}}\right|^{2}+2 \operatorname{Re}\left(\left(\mathcal{M}_{h_{1}}^{*}+\mathcal{M}_{h_{2}}^{*}\right)\left(\mathcal{M}_{\text {cont }}+\mathcal{M}_{\text {cont,loop }}\right)\right)$ \\
\hline $\mathrm{I}\left(h_{2}, \mathrm{C}_{+}+h_{1}\right)$ & $2 \operatorname{Re}\left(\mathcal{M}_{h_{2}}^{*}\left(\mathcal{M}_{\text {cont }}+\mathcal{M}_{\text {cont, loop }}+\mathcal{M}_{\mathrm{h}_{1}}\right)\right)$ \\
\hline$h_{2}+\mathrm{I}\left(\mathrm{C}_{+\circlearrowleft}+h_{1}\right)$ & $\left|\mathcal{M}_{h_{2}}\right|^{2}+2 \operatorname{Re}\left(\mathcal{M}_{h_{2}}^{*}\left(\mathcal{M}_{\text {cont }}+\mathcal{M}_{\text {cont,loop }}+\mathcal{M}_{\mathrm{h}_{1}}\right)\right)$ \\
\hline $\mathrm{I}\left(h_{1}, \mathrm{C}_{+\circlearrowleft}+h_{2}\right)$ & $2 \operatorname{Re}\left(\mathcal{M}_{h_{1}}^{*}\left(\mathcal{M}_{\text {cont }}+\mathcal{M}_{\text {cont,loop }}+\mathcal{M}_{\mathrm{h}_{2}}\right)\right)$ \\
\hline
\end{tabular}

Table 3. Abbreviations used in tables with integrated cross sections and the corresponding mod-squared amplitude expressions. 


\begin{tabular}{|c|c|c|c|}
\hline \multicolumn{4}{|c|}{$\begin{array}{c}g g\left(\rightarrow\left\{H, h_{1}, h_{2}\right\}\right) \rightarrow W^{-} W^{+} \rightarrow \ell \bar{\nu} \bar{\ell}^{\prime} \nu^{\prime} \\
\sigma[\mathrm{fb}], p p, \sqrt{s}=13 \mathrm{TeV} \\
\text { SM and 1HSM (see tables } 1 \text { and } 3)\end{array}$} \\
\hline & \multirow{2}{*}{ SM } & \multicolumn{2}{|c|}{$|\mathcal{M}|^{2}$} \\
\hline & & $\operatorname{Sq}(H)$ & $H+\mathrm{I}(\mathrm{C})$ \\
\hline & $\sigma$ & $13.689(4)$ & $10.420(5)$ \\
\hline & ratio & 1 & $0.7612(5)$ \\
\hline & 1HSM & \multicolumn{2}{|c|}{$|\mathcal{M}|^{2}$} \\
\hline & $M_{h_{2}}[\mathrm{GeV}]$ & $\mathrm{Sq}\left(h_{1+2}\right)$ & $h_{1+2}+\mathrm{I}(\mathrm{C})$ \\
\hline \multirow{8}{*}{$\theta_{1}$} & 700 & $13.090(4)$ & $10.012(5)$ \\
\hline & ratio & 1 & $0.7649(5)$ \\
\hline & 1000 & $13.032(4)$ & $9.934(5)$ \\
\hline & ratio & 1 & $0.7623(5)$ \\
\hline & 1500 & $13.387(4)$ & $10.189(5)$ \\
\hline & ratio & 1 & $0.7611(5)$ \\
\hline & 3000 & $13.619(4)$ & $10.368(5)$ \\
\hline & ratio & 1 & $0.7613(5)$ \\
\hline \multirow{8}{*}{$\theta_{2}$} & 700 & $11.715(4)$ & $9.095(5)$ \\
\hline & ratio & 1 & $0.7763(5)$ \\
\hline & 1000 & $11.503(4)$ & $8.813(5)$ \\
\hline & ratio & 1 & $0.7662(5)$ \\
\hline & 1500 & $12.681(4)$ & $9.651(4)$ \\
\hline & ratio & 1 & $0.7611(4)$ \\
\hline & 3000 & $13.435(4)$ & $10.215(5)$ \\
\hline & ratio & 1 & $0.7603(5)$ \\
\hline
\end{tabular}

Table 4. Cross sections for $g g\left(\rightarrow\left\{H, h_{1}, h_{2}\right\}\right) \rightarrow W^{-} W^{+} \rightarrow \ell \bar{\nu} \bar{\ell}^{\prime} \nu^{\prime}$ in $p p$ collisions at $\sqrt{s}=13 \mathrm{TeV}$ in the Standard Model with $M_{H}=125 \mathrm{GeV}$ and its 1-Higgs-Singlet Extension with $M_{h_{1}}=125 \mathrm{GeV}$, $M_{h_{2}}=700,1000,1500,3000 \mathrm{GeV}$ and mixing angles $\theta_{1}$ and $\theta_{2}$ (see table 1). Mod-squared amplitude contributions are specified using the abbreviations defined in table 3. The ratio $\sigma / \sigma\left(\mathrm{Sq}\left(h_{1+2}\right)\right)$ is also given. The selection cuts in (4.1) are applied. Cross sections are given for a single lepton flavour combination. The integration error is displayed in brackets. 


\begin{tabular}{|c|c|c|c|c|}
\hline & \multicolumn{4}{|c|}{$\begin{array}{c}g g\left(\rightarrow\left\{h_{1}, h_{2}\right\}\right) \rightarrow W^{-} W^{+} \rightarrow \ell \bar{\nu} \bar{\ell}^{\prime} \nu^{\prime} \\
\sigma[\mathrm{fb}], p p, \sqrt{s}=13 \mathrm{TeV} \\
1 \mathrm{HSM}(\text { see tables } 1 \text { and } 3)\end{array}$} \\
\hline & \multirow{2}{*}{$\begin{array}{c}M_{h_{2}} \\
{[\mathrm{GeV}]}\end{array}$} & \multicolumn{3}{|c|}{$|\mathcal{M}|^{2}$} \\
\hline & & $\mathrm{Sq}\left(h_{2}\right)$ & $h_{2}+\mathrm{I}\left(h_{1}\right)$ & $h_{2}+\mathrm{I}\left(\mathrm{C}+h_{1}\right)$ \\
\hline \multirow{8}{*}{$\theta_{1}$} & 700 & $0.07810(2)$ & $0.04113(4)$ & $0.09591(7)$ \\
\hline & ratio & 1 & $0.5266(6)$ & $1.2280(9)$ \\
\hline & 1000 & $0.010824(2)$ & $-0.01621(2)$ & $0.01780(3)$ \\
\hline & ratio & 1 & $-1.498(2)$ & $1.644(2)$ \\
\hline & 1500 & $0.00027818(5)$ & $-0.005749(2)$ & $0.001214(3)$ \\
\hline & ratio & 1 & $-20.668(8)$ & $4.36(1)$ \\
\hline & 3000 & $5.3026(9) \times 10^{-7}$ & $-0.00032008(7)$ & $3.46(2) \times 10^{-5}$ \\
\hline & ratio & 1 & $-603.6(2)$ & $65.2(2)$ \\
\hline \multirow{8}{*}{$\theta_{2}$} & 700 & $0.27776(5)$ & $0.1737(2)$ & $0.3502(2)$ \\
\hline & ratio & 1 & $0.6252(4)$ & $1.2606(7)$ \\
\hline & 1000 & $0.035182(6)$ & $-0.03845(3)$ & $0.06833(5)$ \\
\hline & ratio & 1 & $-1.0928(9)$ & $1.942(2)$ \\
\hline & 1500 & $0.0008885(2)$ & $-0.016227(5)$ & $0.005293(7)$ \\
\hline & ratio & 1 & $-18.262(6)$ & $5.957(8)$ \\
\hline & 3000 & $2.3605(4) \times 10^{-6}$ & $-0.0010870(3)$ & $0.0001561(4)$ \\
\hline & ratio & 1 & $-460.5(2)$ & $66.1(2)$ \\
\hline
\end{tabular}

Table 5. Cross sections for $g g\left(\rightarrow\left\{h_{1}, h_{2}\right\}\right) \rightarrow W^{-} W^{+} \rightarrow \ell \bar{\nu} \bar{\ell}^{\prime} \nu^{\prime}$ in $p p$ collisions at $\sqrt{s}=13 \mathrm{TeV}$ in the 1HSM with focus on heavy Higgs $\left(h_{2}\right)$ production. The ratio $\sigma / \sigma\left(\operatorname{Sq}\left(h_{2}\right)\right)$ is also given. Other details as in table 4. 


\begin{tabular}{|c|c|c|c|c|}
\hline \multicolumn{5}{|c|}{$\begin{array}{c}g g\left(\rightarrow\left\{H, h_{1}, h_{2}\right\}\right) \rightarrow t \bar{t} \rightarrow b \bar{b} \ell \bar{\nu} \bar{\ell}^{\prime} \nu^{\prime} \\
\sigma[\mathrm{fb}], p p, \sqrt{s}=13 \mathrm{TeV} \\
\mathrm{SM} \text { and } 1 \mathrm{HSM}(\text { see tables } 1 \text { and } 3)\end{array}$} \\
\hline & \multirow{2}{*}{$\mathrm{SM}$} & \multicolumn{3}{|c|}{$|\bar{M}|^{2}$} \\
\hline & & $\mathrm{Sq}(H)$ & $H+\mathrm{I}(\mathrm{C})$ & $H+\mathrm{I}\left(\mathrm{C}_{+\circlearrowleft}\right)$ \\
\hline & $\sigma$ & $0.13367(4)$ & $-4.984(2)$ & $10.984(5)$ \\
\hline & ratio & 1 & $-37.28(2)$ & $82.17(5)$ \\
\hline & $1 \mathrm{HSM}$ & \multicolumn{3}{|c|}{$|\mathcal{M}|^{2}$} \\
\hline & $M_{h_{2}}[\mathrm{GeV}]$ & $\mathrm{Sq}\left(h_{1+2}\right)$ & $h_{1+2}+\mathrm{I}(\mathrm{C})$ & $h_{1+2}+\mathrm{I}\left(\mathrm{C}_{+\circlearrowleft}\right)$ \\
\hline \multirow{8}{*}{$\theta_{1}$} & 700 & $0.12834(4)$ & $-4.779(2)$ & $11.203(5)$ \\
\hline & ratio & 1 & $-37.23(2)$ & $87.29(5)$ \\
\hline & 1000 & $0.11820(4)$ & $-4.739(2)$ & $10.605(5)$ \\
\hline & ratio & 1 & $-40.10(2)$ & $89.72(5)$ \\
\hline & 1500 & $0.12735(4)$ & $-4.874(2)$ & $10.759(5)$ \\
\hline & ratio & 1 & $-38.27(2)$ & $84.48(5)$ \\
\hline & 3000 & $0.13228(4)$ & $-4.957(2)$ & $10.932(5)$ \\
\hline & ratio & 1 & $-37.47(2)$ & $82.64(5)$ \\
\hline \multirow{8}{*}{$\theta_{2}$} & 700 & $0.12576(4)$ & $-4.317(2)$ & $11.797(5)$ \\
\hline & ratio & 1 & $-34.33(2)$ & $93.80(5)$ \\
\hline & 1000 & $0.08696(3)$ & $-4.195(2)$ & $9.846(5)$ \\
\hline & ratio & 1 & $-48.24(2)$ & $113.23(6)$ \\
\hline & 1500 & $0.11365(3)$ & $-4.635(2)$ & $10.278(5)$ \\
\hline & ratio & 1 & $-40.78(2)$ & $90.44(5)$ \\
\hline & 3000 & $0.12895(4)$ & $-4.896(2)$ & $10.796(5)$ \\
\hline & ratio & 1 & $-37.97(2)$ & $83.72(5)$ \\
\hline
\end{tabular}

Table 6. Cross sections for $g g\left(\rightarrow\left\{H, h_{1}, h_{2}\right\}\right) \rightarrow t \bar{t} \rightarrow b \bar{b} \ell \bar{\nu} \bar{\ell}^{\prime} \nu^{\prime}$ in $p p$ collisions at $\sqrt{s}=13 \mathrm{TeV}$ in the SM and 1HSM. Virtual corrections $(\circlearrowleft)$ to the continuum background are taken into account (see main text). Other details as in table 4. 


\begin{tabular}{|c|c|c|c|c|c|}
\hline & \multicolumn{5}{|c|}{$\begin{array}{c}g g\left(\rightarrow\left\{h_{1}, h_{2}\right\}\right) \rightarrow t \bar{t} \rightarrow b \bar{b} \ell \bar{\nu} \bar{\ell}^{\prime} \nu^{\prime} \\
\sigma[\mathrm{fb}], p p, \sqrt{s}=13 \mathrm{TeV} \\
1 \mathrm{HSM}(\text { see tables } 1 \text { and } 3)\end{array}$} \\
\hline & \multirow{2}{*}{$\begin{array}{c}M_{h_{2}} \\
{[\mathrm{GeV}]}\end{array}$} & \multicolumn{4}{|c|}{$|\mathcal{M}|^{2}$} \\
\hline & & $\mathrm{Sq}\left(h_{2}\right)$ & $h_{2}+\mathrm{I}\left(h_{1}\right)$ & $h_{2}+\mathrm{I}\left(\mathrm{C}+h_{1}\right)$ & $h_{2}+\mathrm{I}\left(\mathrm{C}_{+\circlearrowleft}+h_{1}\right)$ \\
\hline \multirow{8}{*}{$\theta_{1}$} & 700 & $0.015207(4)$ & $0.00607(1)$ & $-0.00744(2)$ & $0.6966(6)$ \\
\hline & ratio & 1 & $0.3990(7)$ & $-0.489(2)$ & $45.80(4)$ \\
\hline & 1000 & $0.0012148(4)$ & $-0.004079(3)$ & $0.03194(3)$ & $0.09891(8)$ \\
\hline & ratio & 1 & $-3.358(2)$ & $26.30(3)$ & $81.42(7)$ \\
\hline & 1500 & $1.2910(4) \times 10^{-5}$ & $-0.0009172(3)$ & $0.009049(4)$ & $-0.00278(2)$ \\
\hline & ratio & 1 & $-71.05(3)$ & $700.9(4)$ & $-2.15(1) \times 10^{2}$ \\
\hline & 3000 & $7.858(3) \times 10^{-9}$ & $-4.655(2) \times 10^{-5}$ & $0.0005783(2)$ & $-0.0007648(7)$ \\
\hline & ratio & 1 & $-5.923(3) \times 10^{3}$ & $7.359(3) \times 10^{4}$ & $-9.733(9) \times 10^{4}$ \\
\hline \multirow{8}{*}{$\theta_{2}$} & 700 & $0.05395(2)$ & $0.02842(3)$ & $-0.04930(9)$ & $2.436(2)$ \\
\hline & ratio & 1 & $0.5268(5)$ & $-0.914(2)$ & $45.16(4)$ \\
\hline & 1000 & $0.004151(2)$ & $-0.010379(6)$ & $0.07329(9)$ & $0.4855(4)$ \\
\hline & ratio & 1 & $-2.501(2)$ & $17.66(3)$ & $117.0(1)$ \\
\hline & 1500 & $5.566(2) \times 10^{-5}$ & $-0.0026682(8)$ & $0.02068(1)$ & $0.03554(2)$ \\
\hline & ratio & 1 & $-47.94(2)$ & $371.6(3)$ & $638.6(4)$ \\
\hline & 3000 & $8.503(3) \times 10^{-8}$ & $-0.00015896(5)$ & $0.0017632(6)$ & $-0.001246(3)$ \\
\hline & ratio & 1 & $-1869.5(7)$ & $2.0736(9) \times 10^{4}$ & $-1.465(3) \times 10^{4}$ \\
\hline
\end{tabular}

Table 7. Cross sections for $g g\left(\rightarrow\left\{h_{1}, h_{2}\right\}\right) \rightarrow t \bar{t} \rightarrow b \bar{b} \ell \bar{\nu} \bar{\ell}^{\prime} \nu^{\prime}$ in $p p$ collisions at $\sqrt{s}=13 \mathrm{TeV}$ in the 1HSM with focus on heavy Higgs production. Other details as in table 5. 


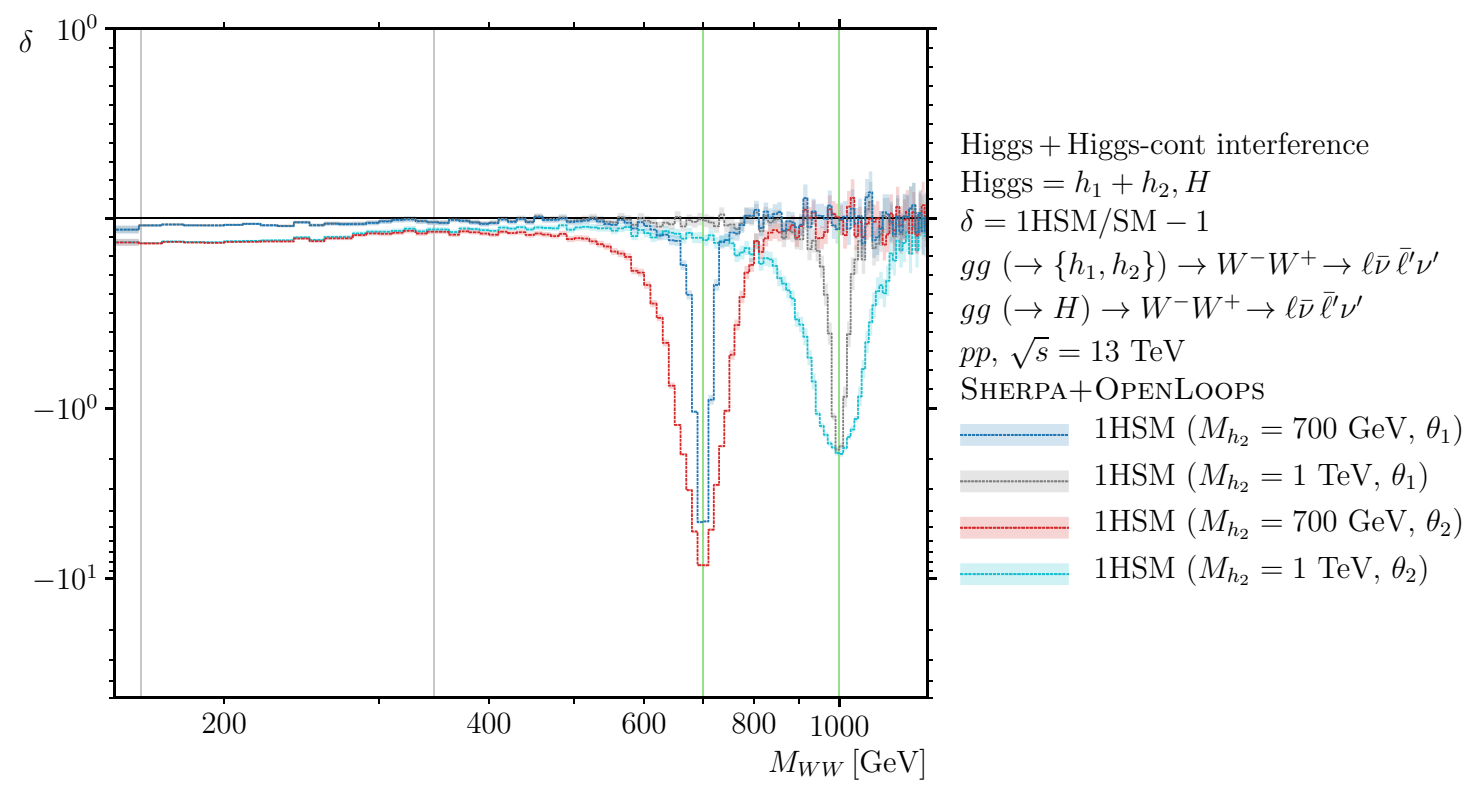

Figure 16. $M_{W W}$ distributions of the relative deviation $\delta=R-1$ of the Higgs cross section including its interference with the background in the $1 \mathrm{HSM}\left(M_{h_{2}}=\{700,1000\} \mathrm{GeV},\left\{\theta_{1}, \theta_{2}\right\}\right)$ compared to the SM for $g g\left(\rightarrow\left\{h_{1}, h_{2}, H\right\}\right) \rightarrow W^{-} W^{+} \rightarrow \ell \bar{\nu} \bar{\ell}^{\prime} \nu^{\prime}$ in $p p$ collisions at $\sqrt{s}=13 \mathrm{TeV}$. $R$ is the ratio of $\sigma\left(h_{1+2}+\mathrm{I}(\mathrm{C})\right)$ to $\sigma(H+\mathrm{I}(\mathrm{C}))$. Other details as in figure 4 .

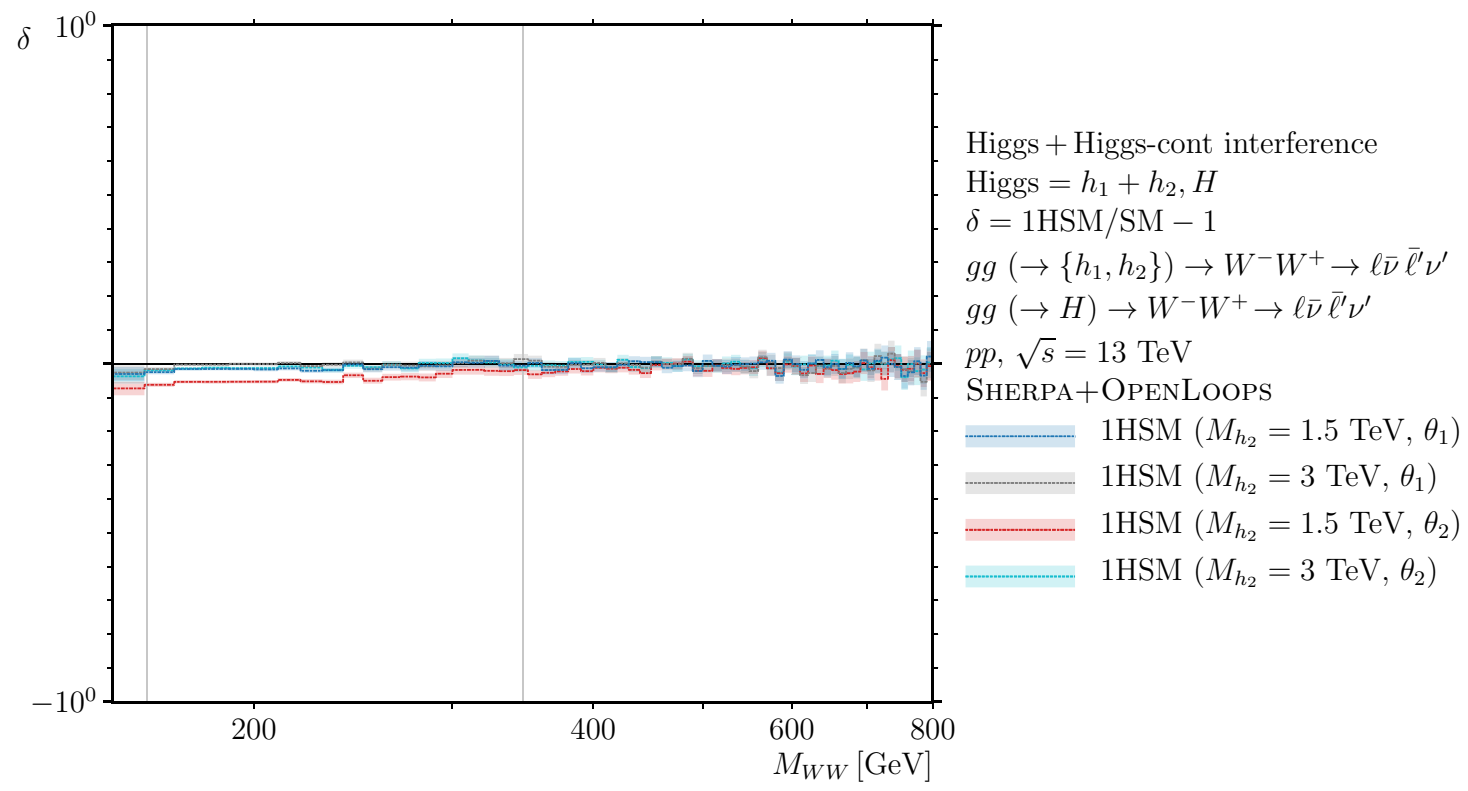

Figure 17. $M_{W W}$ distributions of the relative deviation $\delta=R-1$ of the Higgs cross section including its interference with the background in the $1 \mathrm{HSM}\left(M_{h_{2}}=\{1.5,3\} \mathrm{TeV},\left\{\theta_{1}, \theta_{2}\right\}\right)$ compared to the SM for $g g\left(\rightarrow\left\{h_{1}, h_{2}, H\right\}\right) \rightarrow W^{-} W^{+} \rightarrow \ell \bar{\nu} \bar{\ell}^{\prime} \nu^{\prime}$ in $p p$ collisions at $\sqrt{s}=13 \mathrm{TeV}$. $R$ is the ratio of $\sigma\left(h_{1+2}+\mathrm{I}(\mathrm{C})\right)$ to $\sigma(H+\mathrm{I}(\mathrm{C}))$. Other details as in figure 4. 


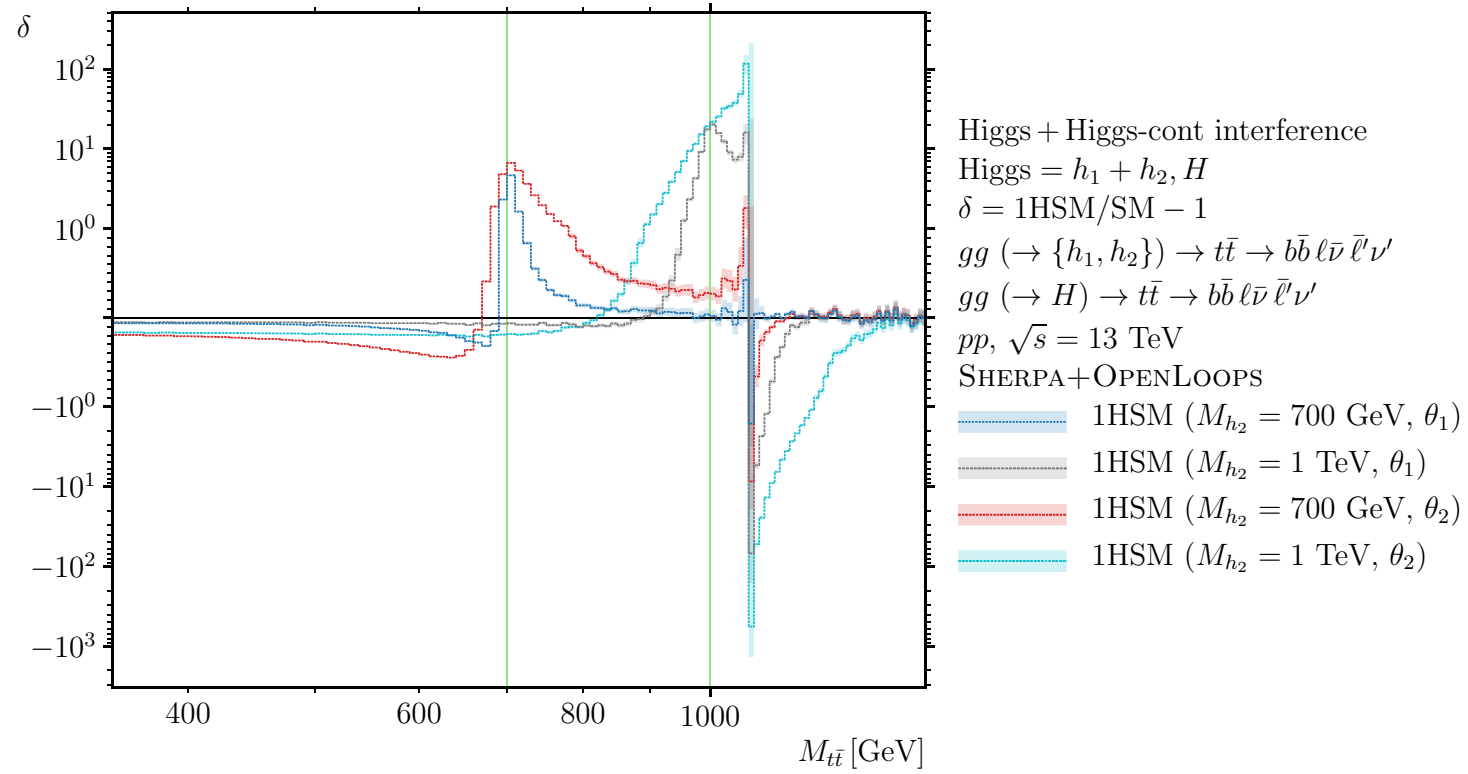

Figure 18. $M_{t \bar{t}}$ distributions of the relative deviation $\delta=R-1$ of the Higgs cross section including its interference with the background in the $1 \mathrm{HSM}\left(M_{h_{2}}=\{700,1000\} \mathrm{GeV},\left\{\theta_{1}, \theta_{2}\right\}\right)$ compared to the SM for $g g\left(\rightarrow\left\{h_{1}, h_{2}, H\right\}\right) \rightarrow t \bar{t} \rightarrow b \bar{b} \ell \bar{\nu} \bar{\ell}^{\prime} \nu^{\prime}$ in $p p$ collisions at $\sqrt{s}=13 \mathrm{TeV} . R$ is the ratio of $\sigma\left(h_{1+2}+\mathrm{I}(\mathrm{C})\right)$ to $\sigma(H+\mathrm{I}(\mathrm{C}))$. Other details as in figure 4.

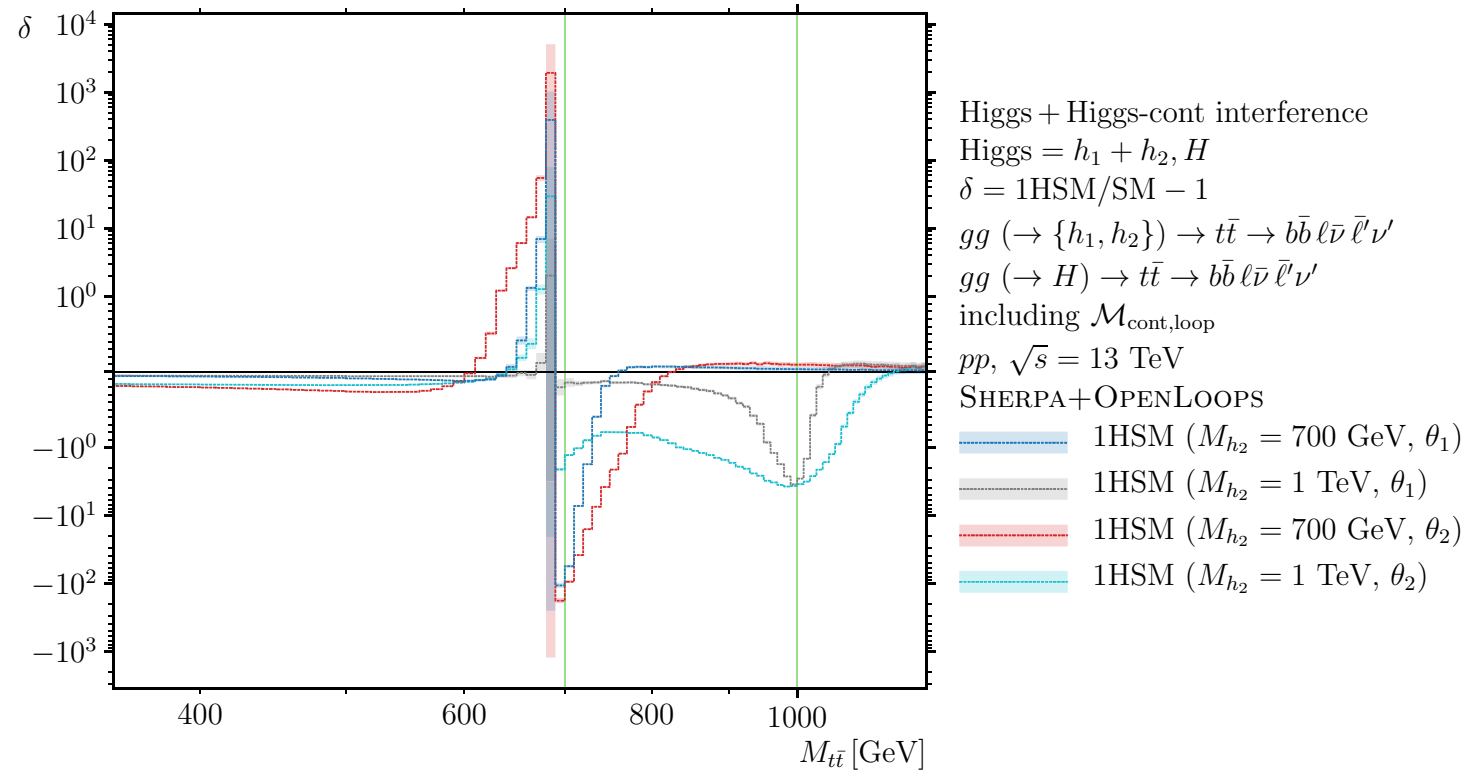

Figure 19. $M_{t \bar{t}}$ distributions of the relative deviation $\delta=R-1$ of the Higgs cross section including its interference with the background in the $1 \mathrm{HSM}\left(M_{h_{2}}=\{700,1000\} \mathrm{GeV},\left\{\theta_{1}, \theta_{2}\right\}\right)$ compared to the SM for $g g\left(\rightarrow\left\{h_{1}, h_{2}, H\right\}\right) \rightarrow t \bar{t} \rightarrow b \bar{b} \ell \bar{\nu} \bar{\ell}^{\prime} \nu^{\prime}$ in $p p$ collisions at $\sqrt{s}=13 \mathrm{TeV} . R$ is the ratio of $\sigma\left(h_{1+2}+\mathrm{I}\left(\mathrm{C}_{+\circlearrowleft}\right)\right)$ to $\sigma\left(H+\mathrm{I}\left(\mathrm{C}_{+\circlearrowleft}\right)\right)$. Other details as in figure 4. 


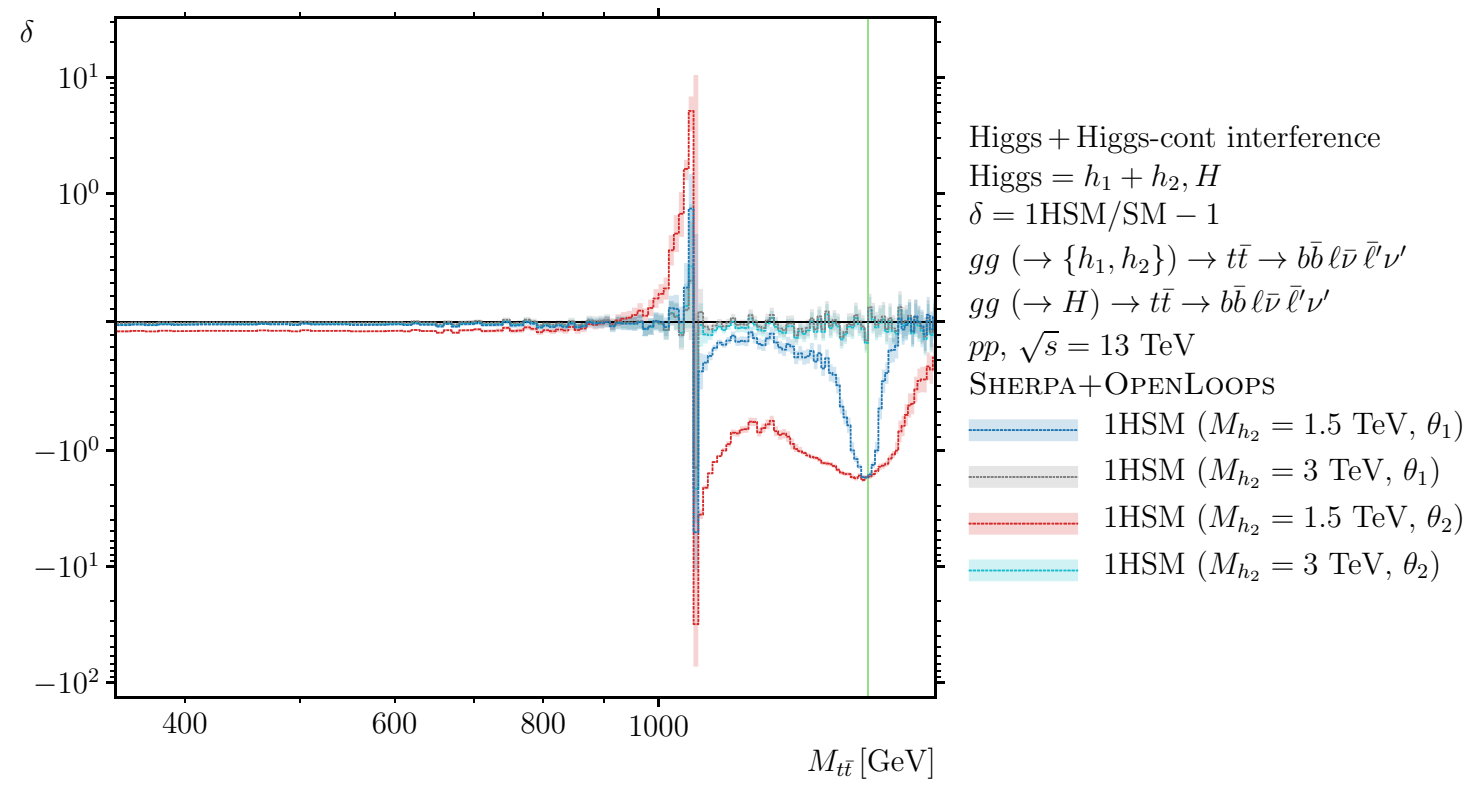

Figure 20. $M_{t \bar{t}}$ distributions of the relative deviation $\delta=R-1$ of the Higgs cross section including its interference with the background in the 1HSM $\left(M_{h_{2}}=\{1.5,3\} \mathrm{TeV},\left\{\theta_{1}, \theta_{2}\right\}\right)$ compared to the $\mathrm{SM}$ for $g g\left(\rightarrow\left\{h_{1}, h_{2}, H\right\}\right) \rightarrow t \bar{t} \rightarrow b \bar{b} \ell \bar{\nu} \bar{\ell}^{\prime} \nu^{\prime}$ in $p p$ collisions at $\sqrt{s}=13 \mathrm{TeV} . R$ is the ratio of $\sigma\left(h_{1+2}+\mathrm{I}(\mathrm{C})\right)$ to $\sigma(H+\mathrm{I}(\mathrm{C}))$. Other details as in figure 4 .

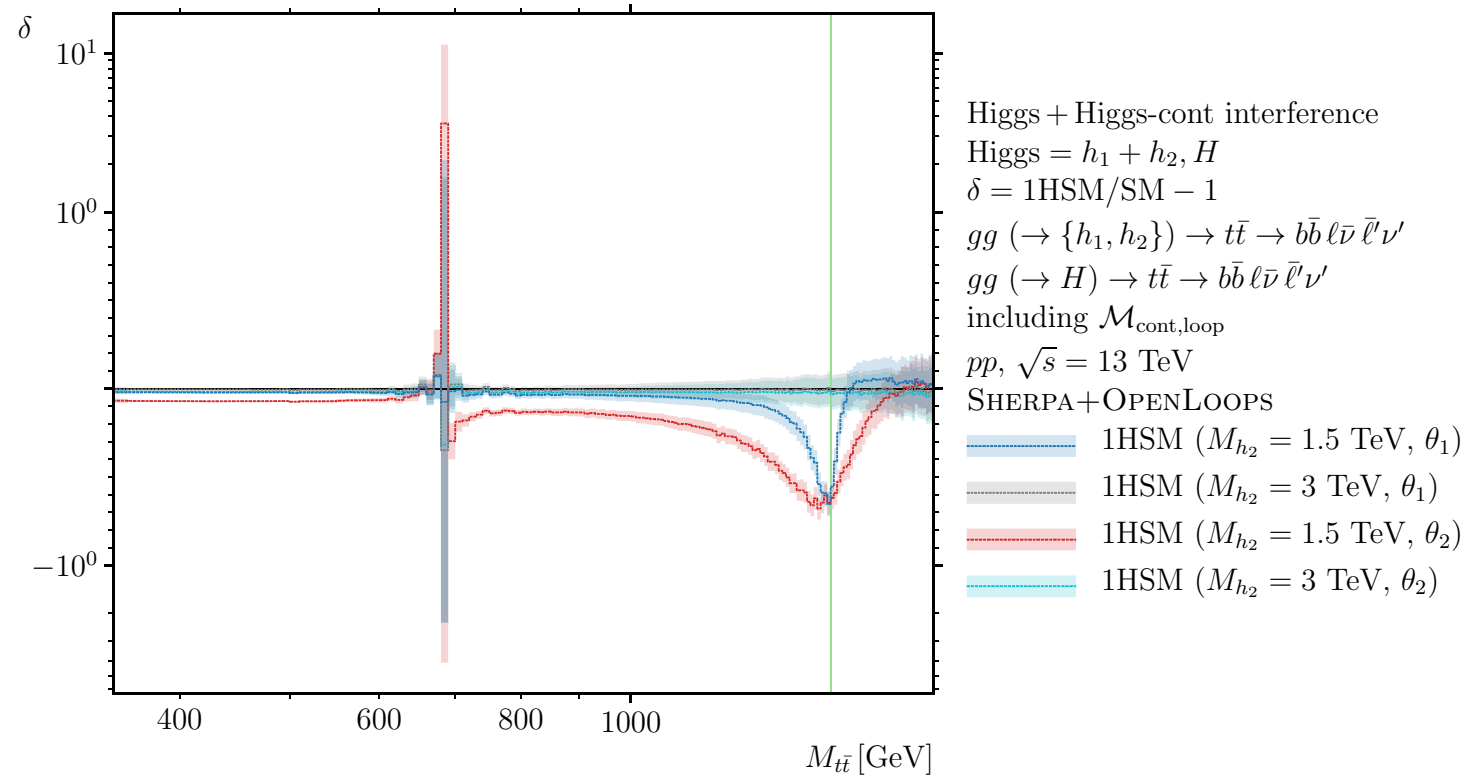

Figure 21. $M_{t \bar{t}}$ distributions of the relative deviation $\delta=R-1$ of the Higgs cross section including its interference with the background in the 1HSM $\left(M_{h_{2}}=\{1.5,3\} \mathrm{TeV},\left\{\theta_{1}, \theta_{2}\right\}\right)$ compared to the SM for $g g\left(\rightarrow\left\{h_{1}, h_{2}, H\right\}\right) \rightarrow t \bar{t} \rightarrow b \bar{b} \ell \bar{\nu} \bar{\ell}^{\prime} \nu^{\prime}$ in $p p$ collisions at $\sqrt{s}=13 \mathrm{TeV} . R$ is the ratio of $\sigma\left(h_{1+2}+\mathrm{I}\left(\mathrm{C}_{+\circlearrowleft}\right)\right)$ to $\sigma\left(H+\mathrm{I}\left(\mathrm{C}_{+\circlearrowleft}\right)\right)$. Other details as in figure 4. 


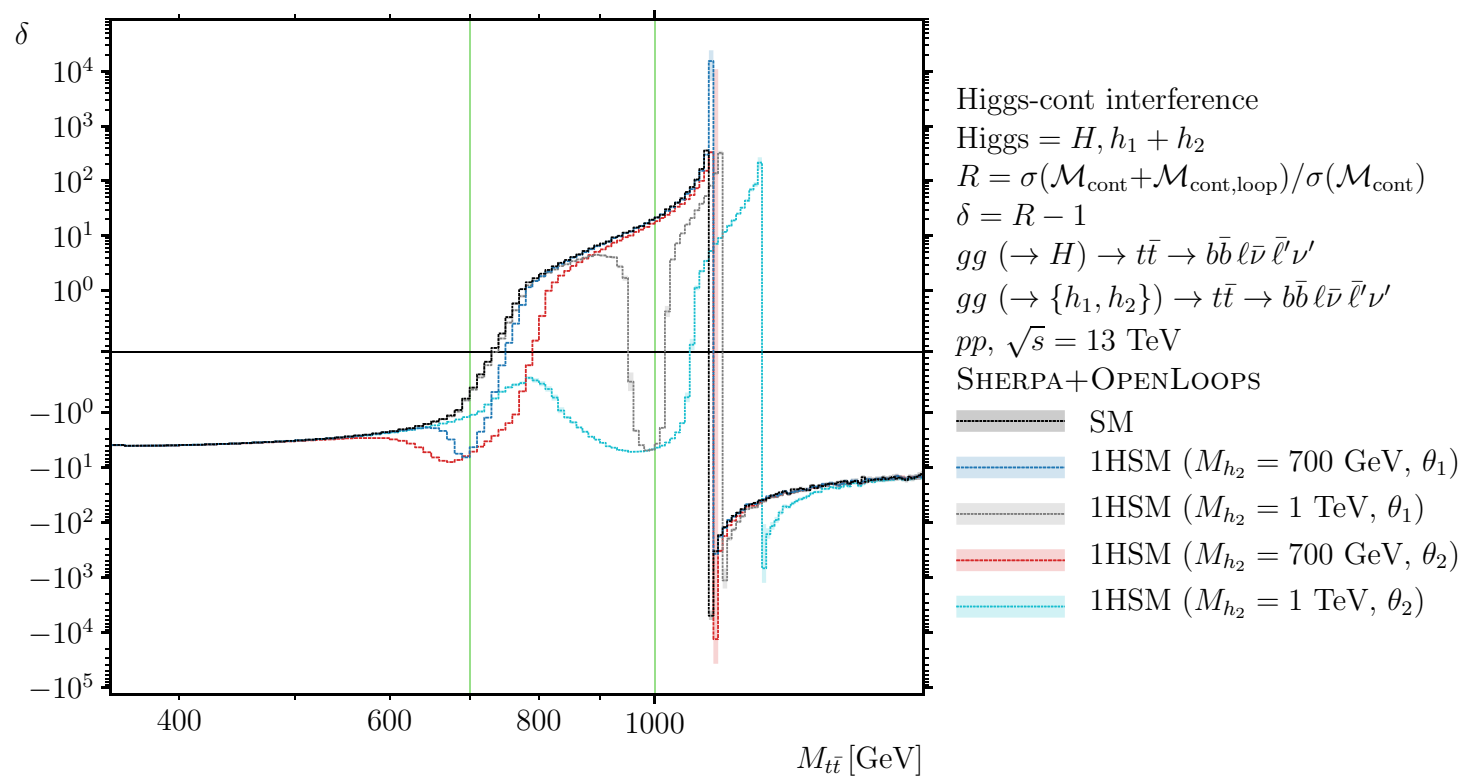

Figure 22. $\quad M_{t \bar{t}}$ distributions of the relative deviation $\delta=R-1$ of the Higgs interference with the background without and with the virtual corrections $\left(\mathcal{M}_{\text {cont,loop }}\right)$ in the SM and 1HSM $\left(M_{h_{2}}=\{700,1000\} \mathrm{GeV},\left\{\theta_{1}, \theta_{2}\right\}\right)$ for $g g\left(\rightarrow\left\{h_{1}, h_{2}, H\right\}\right) \rightarrow t \bar{t} \rightarrow b \bar{b} \ell \bar{\nu} \bar{\ell}^{\prime} \nu^{\prime}$ in $p p$ collisions at $\sqrt{s}=13 \mathrm{TeV} . R$ is the ratio of $\sigma\left(\mathrm{I}\left(H, \mathrm{C}_{+} \circlearrowleft\right)\right)$ to $\sigma(\mathrm{I}(H, \mathrm{C}))$ and $\sigma\left(\mathrm{I}\left(h_{1+2}, \mathrm{C}_{+\circlearrowleft}\right)\right)$ to $\sigma\left(\mathrm{I}\left(h_{1+2}, \mathrm{C}\right)\right)$ in the SM and 1HSM, respectively. Other details as in figure 4.

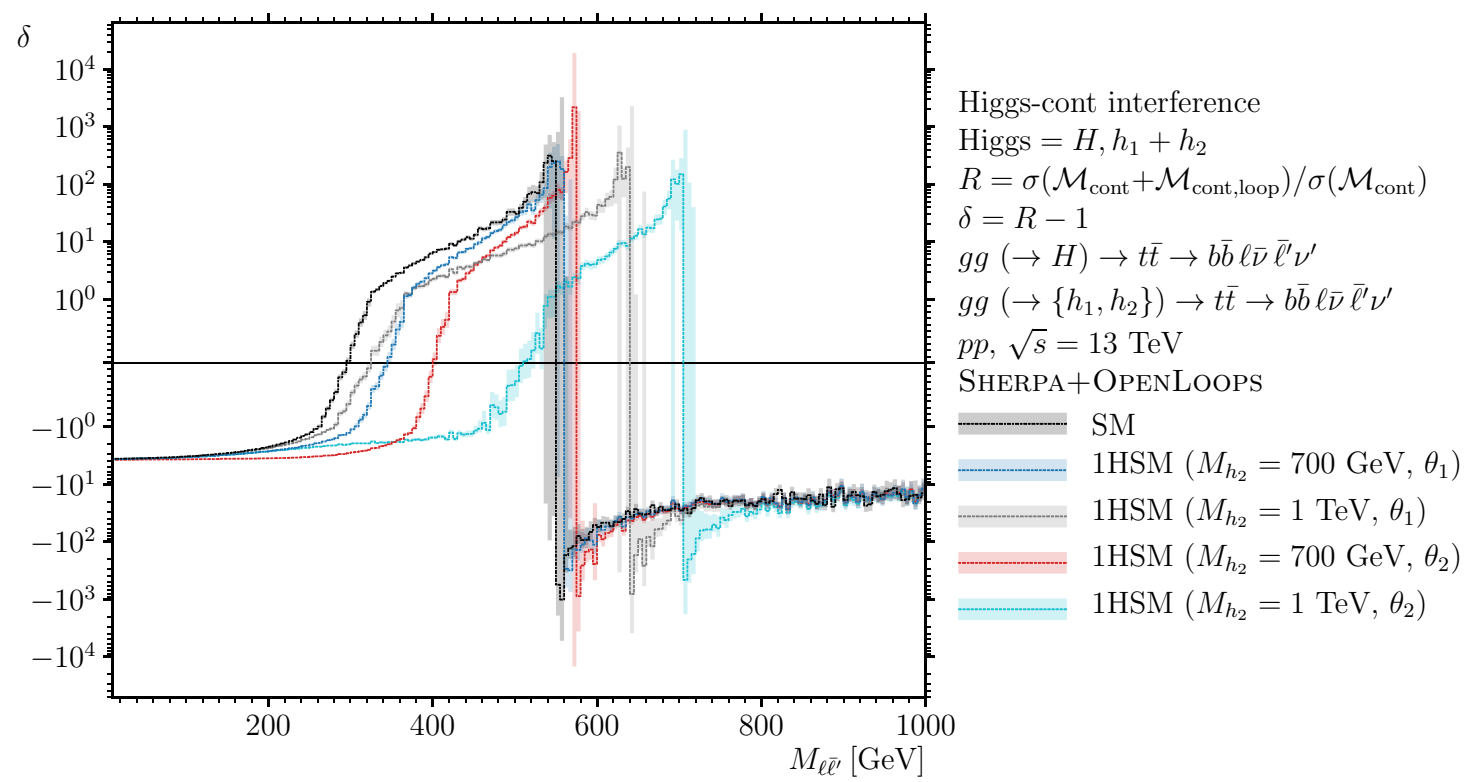

Figure 23. $M_{\ell \bar{\ell}^{\prime}}$ distributions of the relative deviation $\delta=R-1$ of the Higgs interference with the background without and with the virtual corrections in the SM and 1HSM $\left(M_{h_{2}}=\{700,1000\} \mathrm{GeV}\right.$, $\left.\left\{\theta_{1}, \theta_{2}\right\}\right)$ for $g g\left(\rightarrow\left\{H, h_{1}, h_{2}\right\}\right) \rightarrow t \bar{t} \rightarrow b \bar{b} \ell \bar{\nu} \bar{\ell}^{\prime} \nu^{\prime}$ in $p p$ collisions at $\sqrt{s}=13 \mathrm{TeV}$. Other details as in figure 22 . 


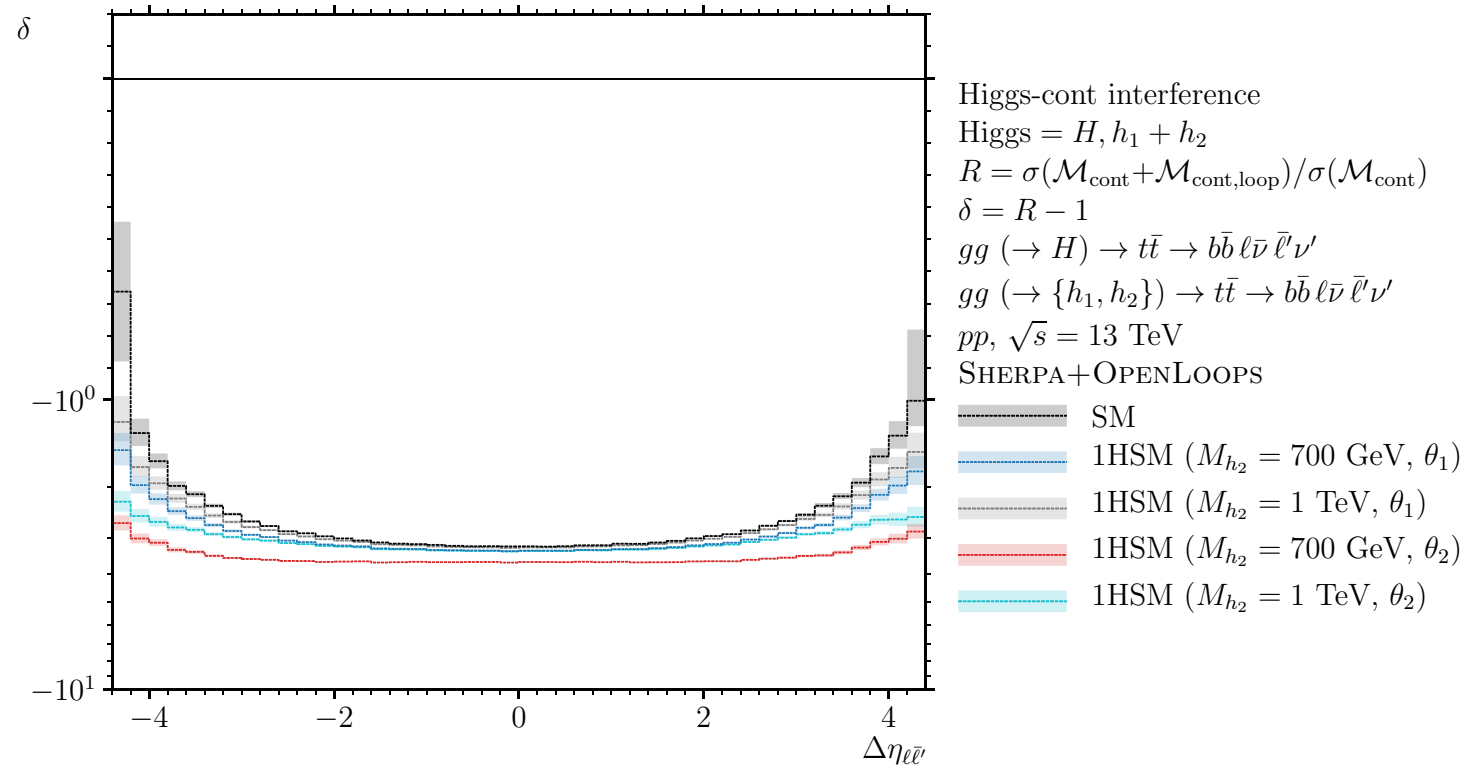

Figure 24. $\Delta \eta_{\ell \bar{\ell}^{\prime}}$ distributions of the relative deviation $\delta=R-1$ of the Higgs interference with the background without and with the virtual corrections in the SM and 1HSM $\left(M_{h_{2}}=\{700,1000\} \mathrm{GeV}\right.$, $\left.\left\{\theta_{1}, \theta_{2}\right\}\right)$ for $g g\left(\rightarrow\left\{H, h_{1}, h_{2}\right\}\right) \rightarrow t \bar{t} \rightarrow b \bar{b} \ell \bar{\nu} \bar{\ell}^{\prime} \nu^{\prime}$ in $p p$ collisions at $\sqrt{s}=13 \mathrm{TeV}$. Other details as in figure 22 .

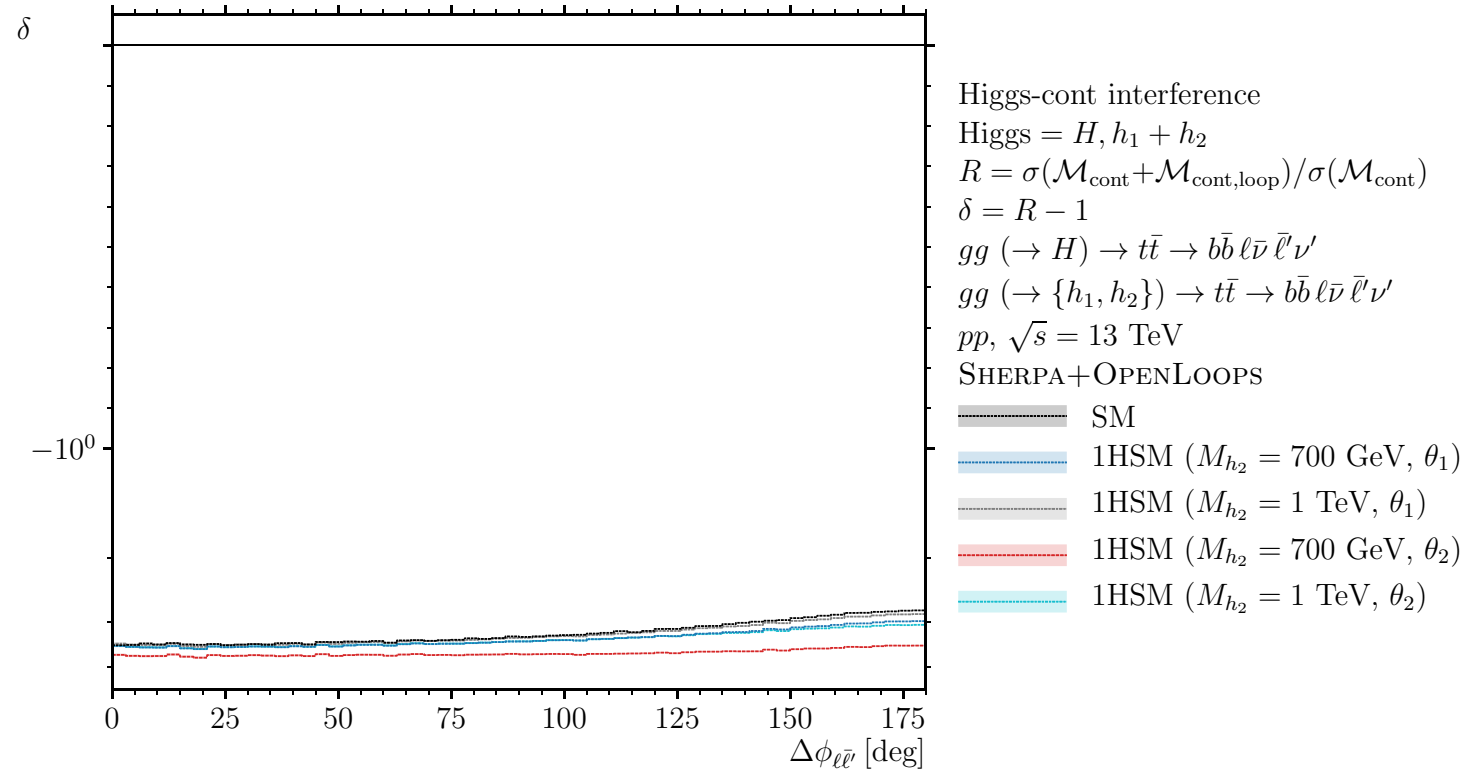

Figure 25. $\Delta \phi_{\ell \bar{\ell}^{\prime}}$ distributions of the relative deviation $\delta=R-1$ of the Higgs interference with the background without and with the virtual corrections in the SM and $1 \mathrm{HSM}\left(M_{h_{2}}=\{700,1000\} \mathrm{GeV}\right.$, $\left.\left\{\theta_{1}, \theta_{2}\right\}\right)$ for $g g\left(\rightarrow\left\{H, h_{1}, h_{2}\right\}\right) \rightarrow t \bar{t} \rightarrow b \bar{b} \ell \bar{\nu} \bar{\ell}^{\prime} \nu^{\prime}$ in $p p$ collisions at $\sqrt{s}=13 \mathrm{TeV}$. Other details as in figure 22 . 


\section{Discussion}

The $\mathrm{Sq}\left(h_{1+2}\right)$ integrated cross sections displayed in table 4 for $W W$ production in the 1HSM exhibit a relatively small deviation from the $\mathrm{SM}$ cross section $\mathrm{Sq}(H)$, which ranges from $-5 \%$ to $-0.05 \%$ for $M_{h_{2}}=1 \mathrm{TeV}$ and $M_{h_{2}}=3 \mathrm{TeV}$, respectively, and the mixing angle $\theta_{1}\left(\theta_{1} \lesssim 0.2\right.$, see table 1$)$. Increasing, for illustration, ${ }^{18}$ the mixing angle to $\theta_{2} \lesssim 0.4$, the cross section deviation range increases to $-16 \%$ to $-2 \%$ with corresponding heavy Higgs masses. When adding the continuum background interference, in the 1HSM and SM the cross section is reduced uniformly by a factor close to 0.76 . Since in table 4 the $\left|\mathcal{M}_{h_{1}}+\mathcal{M}_{h_{2}}\right|^{2}$ and $\left|\mathcal{M}_{H}\right|^{2}$ Higgs cross sections are compared, due to unitarity constraints it is not surprising that cross section deviations are small and the impact of the interference is uniform.

In table 5 , we show for $W W$ production how interference affects the integrated heavy Higgs resonance cross section $\mathrm{Sq}\left(h_{2}\right)$. Due to the falling gluon PDF and the decreasing value of $\theta_{1,2}$ for $M_{h_{2}}=\{1.5,3\} \mathrm{TeV}$ (see table 1 ), $\mathrm{Sq}\left(h_{2}\right)$ decreases rapidly with increasing $M_{h_{2}}$ and, as expected, is roughly a factor $3-5$ higher for the mixing angle $\theta_{2}<\pi / 4$, which is larger than $\theta_{1}>0$. The heavy Higgs cross section $\operatorname{Sq}\left(h_{2}\right)$ is drastically altered when taking into account the interference with the light Higgs $\mathrm{I}\left(h_{1}, h_{2}\right)$, because the light Higgs cross section $\mathrm{Sq}\left(h_{1}\right)$ is significantly larger than $\mathrm{Sq}\left(h_{2}\right)$ throughout (see table 10 in appendix B). As seen in table 5 , the cross section ratio $\left(h_{2}+\mathrm{I}\left(h_{1}\right)\right) / \mathrm{Sq}\left(h_{2}\right)$ ranges from $0.527(0.625)$ to $-604(-461)$ when $M_{h_{2}}$ increases from $700 \mathrm{GeV}$ to $3 \mathrm{TeV}$ with mixing angle $\theta_{1}\left(\theta_{2}\right)$. When comparing the integrated cross sections $\mathrm{Sq}\left(h_{2}\right), h_{2}+\mathrm{I}\left(h_{1}\right)$ and $h_{2}+\mathrm{I}\left(\mathrm{C}+h_{1}\right)$, where the heavy Higgs-continuum background interference has also been added in the third quantity, it is apparent that the heavy Higgs-light Higgs interference $\mathrm{I}\left(h_{1}, h_{2}\right)$ and $\mathrm{I}\left(h_{1}, \mathrm{C}\right)$ always have opposite signs (see also table 10 in appendix B), which results in a substantial reduction of the interference impact on the heavy resonance in $W W$ production. This can be seen in table 5: the cross section ratio $\left(h_{2}+\mathrm{I}\left(\mathrm{C}+h_{1}\right)\right) / \mathrm{Sq}\left(h_{2}\right)$ only ranges from 1.228 (1.261) to 65 (66) when $M_{h_{2}}$ increases from $700 \mathrm{GeV}$ to $3 \mathrm{TeV}$ with mixing angle $\theta_{1}\left(\theta_{2}\right)$.

Analogous results, but for $t \bar{t}$ production extended with the one-loop $(\circlearrowleft)$ continuum background amplitude, are presented in tables 6 and 7 . The $\mathrm{Sq}\left(h_{1+2}\right)$ integrated cross sections in the 1HSM displayed in table 6 exhibit a deviation from the SM cross section $\mathrm{Sq}(H)$ ranging from $-12 \%(-35 \%)$ to $-1 \%(-4 \%)$ for $M_{h_{2}}=1 \mathrm{TeV}$ and $M_{h_{2}}=3 \mathrm{TeV}$, respectively, and the mixing angle $\theta_{1}\left(\theta_{2}\right)$. We note that the extreme deviations occur for the same values of $M_{h_{2}}$ for $t \bar{t}$ and $W W$ production and that the deviations are larger in $t \bar{t}$ than in $W W$ production. When adding the interference with the tree-level continuum background, in the 1HSM and SM the cross section changes by a factor of approximately -40 , i.e. the interference at tree level is negative and about 40 times larger than the heavy resonance cross section. When the interference with the one-loop continuum background is included, the result changes sign and is at least twice as large. This implies that the interference with the one-loop continuum background is at least three times larger than the tree-level interference, with opposite sign. Already at the integrated cross section level,

\footnotetext{
${ }^{18}$ Note that $\theta_{2}$ is no longer compatible with experimental bounds.
} 
it is therefore important to include all one-loop contributions to obtain reliable signal plus interference results.

In table 7 , we show for $t \bar{t}$ production how interference affects the heavy Higgs cross section $\mathrm{Sq}\left(h_{2}\right)$. As before, $\mathrm{Sq}\left(h_{2}\right)$ decreases rapidly with increasing $M_{h_{2}}$ and, as expected, is roughly a factor 3-10 higher for $\theta_{2}$ than for $\theta_{1}$. The heavy Higgs cross section $\operatorname{Sq}\left(h_{2}\right)$ is substantially or even drastically altered when taking into account the interference with the light Higgs $\mathrm{I}\left(h_{1}, h_{2}\right)$, because the $t \bar{t}$ light Higgs cross section $\mathrm{Sq}\left(h_{1}\right)$ is much larger than $\mathrm{Sq}\left(h_{2}\right)$ (see table 12 in appendix $\mathrm{B}$ ). The cross section ratio $\left(h_{2}+\mathrm{I}\left(h_{1}\right)\right) / \mathrm{Sq}\left(h_{2}\right)$ ranges from $0.400(0.527)$ to $-5.92 \times 10^{3}(-1870)$ when $M_{h_{2}}$ increases from $700 \mathrm{GeV}$ to $3 \mathrm{TeV}$ with mixing angle $\theta_{1}\left(\theta_{2}\right)$, where the ratio is negative for $M_{h_{2}} \gtrsim 1 \mathrm{TeV}$. When comparing the integrated cross sections $\mathrm{Sq}\left(h_{2}\right), h_{2}+\mathrm{I}\left(h_{1}\right)$ and $h_{2}+\mathrm{I}\left(\mathrm{C}+h_{1}\right)$, where the heavy Higgs(tree-level-)continuum background interference has been added in the third quantity, it is apparent that the heavy Higgs-light Higgs interference $\mathrm{I}\left(h_{1}, h_{2}\right)$ and $\mathrm{I}\left(h_{2}, \mathrm{C}\right)$ typically have opposite signs (see also table 12 in appendix B). In contrast to $W W$ production, despite the opposite sign, the result is a strong increase of the interference impact on the heavy resonance for $M_{h_{2}} \gtrsim 1 \mathrm{TeV}$. As seen in table 7 , the cross section ratio $\left(h_{2}+\mathrm{I}\left(\mathrm{C}+h_{1}\right)\right) / \mathrm{Sq}\left(h_{2}\right)$ ranges from $26.3(17.6)$ to $7.36 \times 10^{4}\left(2.07 \times 10^{4}\right)$ when $M_{h_{2}}$ increases from 1 to $3 \mathrm{TeV}$ with mixing angle $\theta_{1}\left(\theta_{2}\right)$. Furthermore, the rightmost column of table 7 demonstrates that it is essential to take into account the interference with the one-loop continuum background. The cross section ratio $\left(h_{2}+\mathrm{I}\left(\mathrm{C}_{+}+h_{1}\right)\right) / \mathrm{Sq}\left(h_{2}\right)$ ranges from $45.80(45.2)$ to $-9.7 \times 10^{4}$ $\left(-1.47 \times 10^{4}\right)$ when $M_{h_{2}}$ increases from 1 to $3 \mathrm{TeV}$ with mixing angle $\theta_{1}\left(\theta_{2}\right)$. In all studied cases, the inclusion of the one-loop continuum background changes the cross section substantially or even drastically.

Additional insight is gained by studying differential distributions. For $W W$ production, the $M_{W W}$ distribution shown in figure 4 illustrates several characteristics. First, as expected, $d \sigma\left(\left|\mathcal{M}_{h_{1}}\right|^{2}\right)$ resembles $d \sigma\left(\left|\mathcal{M}_{H}\right|^{2}\right)$ (see figure 26). Comparing the same figures, one also finds that, as expected, $d \sigma\left(2 \operatorname{Re}\left(\mathcal{M}_{h_{1}}^{*} \mathcal{M}_{\text {cont }}\right)\right)$ and $d \sigma\left(2 \operatorname{Re}\left(\mathcal{M}_{H}^{*} \mathcal{M}_{\text {cont }}\right)\right)$ have the same shape. Secondly, figure 4 illustrates that $d \sigma\left(2 \operatorname{Re}\left(\mathcal{M}_{h_{2}}^{*} \mathcal{M}_{\text {cont }}\right)\right)$ and $d \sigma\left(2 \operatorname{Re}\left(\mathcal{M}_{h_{2}}^{*} \mathcal{M}_{h_{1}}\right)\right)$ have opposite sign behaviour with respect to $M_{W W}=M_{h_{2}}$. Furthermore, the sign behaviour of $d \sigma\left(2 \operatorname{Re}\left(\mathcal{M}_{h_{1 / 2}}^{*} \mathcal{M}_{\text {cont }}\right)\right)$ at $M_{W W}=M_{h_{1 / 2}}$ is identical to the sign behaviour of $d \sigma\left(2 \operatorname{Re}\left(\mathcal{M}_{H}^{*} \mathcal{M}_{\text {cont }}\right)\right)$ at $M_{W W}=M_{H}$, which is dictated by unitarity cancellations at high energy. $d \sigma\left(2 \operatorname{Re}\left(\mathcal{M}_{h_{2}}^{*}\left(\mathcal{M}_{\text {cont }}+\mathcal{M}_{\mathrm{h}_{1}}\right)\right)\right)$ illustrates the compensation between the two types of interference for the heavy resonance, which was discussed for table 5 above, at the differential level. We note the strong interference reduction in the vicinity of the $h_{2}$ peak. But, the mitigating effect of the heavy Higgs-light Higgs interference decreases steadily down to the $W W$ threshold. The $M_{T, W W}$ distribution shown in figure 5 is related to the $M_{W W}$ distribution discussed above by the fact that $M_{T, W W} \leq M_{W W}$ is guaranteed for the $M_{T, W W}$ definition used here. The $M_{\ell \bar{\ell}^{\prime}}$ distribution displayed in figure 6 demonstrates that the interference impact for the heavy Higgs signal is largest for $M_{\ell \bar{\ell}^{\prime}} \lesssim 150 \mathrm{GeV}$ and decreases continuously for higher dilepton invariant masses. The $\Delta \eta_{\bar{\ell} \bar{\ell}^{\prime}}, \Delta \phi_{\ell \overline{\ell^{\prime}}}$ and $\Delta R_{\bar{\ell}_{\ell^{\prime}}}$ distributions displayed in figures 7, 8 and 9, respectively, illustrate that the interference impact for the heavy Higgs signal is large except for approximately back-to-back dilepton configurations. As small dilepton opening angles are typically selected in Higgs $\rightarrow W W$ 
searches [101], this implies that the angular dependence of interference effects is important and should be taken into account in such studies.

For $t \bar{t}$ production, the $M_{t \bar{t}}$ and $M_{T, W W}$ distributions are shown in figures 10 and 11, respectively. Comparing the $M_{t \bar{t}}$ distributions in the 1HSM (figure 10) and the SM (figure 28, see also figure 29) yields: first, in analogy to $W W$ production, shape agreement is found when $h_{1}$-dependent $1 \mathrm{HSM}$ cross sections are compared with the corresponding $H$-dependent SM cross sections. Secondly, the same pattern for the sign behaviour of $d \sigma\left(2 \operatorname{Re}\left(\mathcal{M}_{h_{2}}^{*} \mathcal{M}_{\text {cont }}\right)\right)$ and $d \sigma\left(2 \operatorname{Re}\left(\mathcal{M}_{h_{2}}^{*} \mathcal{M}_{h_{1}}\right)\right)$ is found as in $W W$ production. As new feature, the typically dominant impact of the one-loop continuum background amplitude

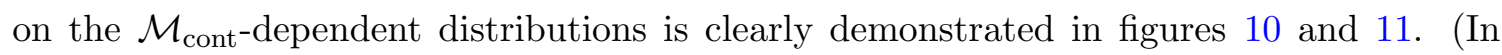
figures 28 and 29, the same is demonstrated for the corresponding SM distributions.) In these figures and all other $t \bar{t}$ distributions, it is apparent that interference is the leading cross section contribution and the Higgs resonance cross section is subleading. The $M_{\ell \bar{\ell}^{\prime}}$, $\Delta \eta_{\ell \overline{\ell^{\prime}}}, \Delta \phi_{\ell \bar{\ell}^{\prime}}$ and $\Delta R_{\ell \bar{\ell}^{\prime}}$ distributions displayed in figures 12, 13, 14 and 15, respectively, confirm both statements for the dilepton invariant mass and angular observables.

Employing the Higgs invariant mass distribution and considering all benchmark points, for $W W$ production in figures 16 and 17 and for $t \bar{t}$ production without and with one-loop continuum background amplitude in figures 18 and 20 and figures 19 and 21, respectively, the relative deviation of the Higgs cross section in the 1HSM and SM $\left(\left|\mathcal{M}_{h_{1}}+\mathcal{M}_{h_{2}}\right|^{2}\right.$, $\left.\left|\mathcal{M}_{H}\right|^{2}\right)$ including interference with the continuum background is shown. Near the heavy Higgs resonance and in extended neighbouring regions the deviation can be $\mathcal{O}(100 \%)$ or even significantly larger. Elsewhere, the deviation is $\mathcal{O}(10 \%)$.

Similarly, for $t \bar{t}$ production in the SM and 1 HSM various distributions shown in figures 22, 23, 24 and 25 illustrate the relative deviation of the interference cross section without and with one-loop continuum background amplitude at the differential level. For $M_{t \bar{t}}$ and $M_{\ell \bar{\ell}^{\prime}}$ the deviation significantly exceeds $100 \%$ in large invariant mass regions. For $\Delta \eta_{\ell \bar{\ell}^{\prime}}$ and $\Delta \phi_{\ell \bar{\ell}^{\prime}}$ the deviation is $\mathcal{O}(2-4)$ and its differential variation is non-negligible, but less pronounced.

\section{Conclusions}

A detailed study of Higgs interference effects at the one-loop level in the 1HSM was presented for the $W W$ and $t \bar{t}$ decay modes with fully leptonic $W W$ decay. We calculated with massive top and bottom quarks and explored interference effects for benchmark points with a heavy Higgs mass that significantly exceeds $2 m_{t}$. More specifically, the $M_{h_{2}}$ range $700-$ $3000 \mathrm{GeV}$ was studied with corresponding mixing angles compatible with current limits as well as a second set of mixing angles, roughly twice as large, to illustrate the dependence on the mixing angle. In the $W W$ channel, the Higgs signal and the interfering continuum background are loop induced. In the $t \bar{t}$ channel, which features a tree-level background, we also calculated the interference with the one-loop background (applying the NWA to $t$ and $\bar{t}$, which, due to the appearance of the absorptive part, was found to dominate the studied distributions. More generally, our results indicate that NLO interference contributions substantially change the normalisation and shape of BSM and SM differential 
Higgs cross section distributions in invariant-mass as well as angular kinematic variables. This can be understood via the appearance of a non-trivial phase that is caused by loop corrections to the continuum background. Full NLO corrections are therefore essential and, when available, should be taken into account in all interference-affected experimental searches for heavy Higgs resonances. We conjecture that the same applies to searches for other heavy resonances. As corollary, we find that the commonly used geometric average $K$-factor approximation $K_{\text {interference }} \approx\left(K_{\text {Higgs }} K_{\text {background }}\right)^{1 / 2}$ is not appropriate.

Finally, we note that our 1HSM and SM implementation in SherPA+OpenLoops, which can be used as parton-level integrator or event generator, is included in the arXiv submission as ancillary file. Supplementary figures with distributions for all studied quantities, models and benchmark points are available as Web download.

\section{Acknowledgments}

We are grateful to Silvan Kuttimalai and Stefan Höche for useful suggestions and SHERPA and MatPlotliB support. N.K. would like to thank CERN for hospitality and partial financial support through the CERN Theory Institute on LHC and the Standard Model: Physics and Tools and the general assembly meetings of the LHC Higgs Cross Section Working Group. N.K. is also grateful to C. Anastasiou and V. Hirschi for hospitality at ETH Zurich as well as access to the RHUL Physics HPC cluster. A.L. would like to thank the Department of Physics, Royal Holloway, University of London, for supplementary financial support and the Theoretical Particle Physics Group, University of Sussex, for hospitality and access to their computer cluster. This work was supported in part by STFC grant ST/P000738/1 and by the U.S. Department of Energy under contract number DE-AC0276SF00515. W.S. has received funding from the European Union's Horizon 2020 research and innovation programme under the Marie Skłodowska-Curie grant agreement (GA) No 665593 awarded to the Science and Technology Facilities Council. Feynman graphs were drawn with JAXODRAW [103]. We are grateful to J. Collins for providing a remedy for a known JaxoDraw flaw. Plots were created with Rivet [102] and Matplotlib [104].

\section{A Partial decay widths for $h_{2} \rightarrow h_{1} h_{1}, h_{1} h_{1} h_{1}, h_{1} h_{1} h_{1} h_{1}$}

The partial decay widths for $h_{2} \rightarrow h_{1} h_{1}, h_{2} \rightarrow h_{1} h_{1} h_{1}$ and $h_{2} \rightarrow h_{1} h_{1} h_{1} h_{1}$ for the considered benchmark points are given in table $8 .{ }^{19}$

\footnotetext{
${ }^{19}$ The absorptive part of the occurring $h_{2}$ propagators, which due to the kinematic constraints cannot be on-shell, was neglected. We checked, by iterating once with the obtained values for $\Gamma_{h_{2}}$, that the resulting uncertainty ranges from $0.05 \%$ to $5 \%$, depending on the benchmark point, and is hence generally small.
} 


\begin{tabular}{|l|c|c|c|}
\hline \multicolumn{4}{|c|}{$\theta=\theta_{1}$} \\
\hline$M_{h_{2}}[\mathrm{GeV}]$ & $\Gamma\left(h_{2} \rightarrow h_{1} h_{1}\right)[\mathrm{GeV}]$ & $\Gamma\left(h_{2} \rightarrow h_{1} h_{1} h_{1}\right)[\mathrm{GeV}]$ & $\Gamma\left(h_{2} \rightarrow h_{1} h_{1} h_{1} h_{1}\right)[\mathrm{GeV}]$ \\
\hline 700 & $2.1556(1)$ & $0.00468(2)$ & $6.24(4) \times 10^{-7}$ \\
\hline 1000 & $6.0953(1)$ & $0.1692(7)$ & $0.001718(9)$ \\
\hline 1500 & $9.8911(1)$ & $0.218(2)$ & $0.001632(8)$ \\
\hline 3000 & $20.658(1)$ & $0.306(2)$ & $0.001060(7)$ \\
\hline
\end{tabular}

\begin{tabular}{|l|c|c|c|}
\hline \multicolumn{4}{|c|}{$\theta=\theta_{2}$} \\
\hline$M_{h_{2}}[\mathrm{GeV}]$ & $\Gamma\left(h_{2} \rightarrow h_{1} h_{1}\right)[\mathrm{GeV}]$ & $\Gamma\left(h_{2} \rightarrow h_{1} h_{1} h_{1}\right)[\mathrm{GeV}]$ & $\Gamma\left(h_{2} \rightarrow h_{1} h_{1} h_{1} h_{1}\right)[\mathrm{GeV}]$ \\
\hline 700 & $4.1798(1)$ & $0.507(2)$ & $0.01451(8)$ \\
\hline 1000 & $11.604(1)$ & $7.34(4)$ & $2.46(2)$ \\
\hline 1500 & $27.26(1)$ & $12.9(2)$ & $3.91(2)$ \\
\hline 3000 & $66.8(1)$ & $21.4(2)$ & $4.17(2)$ \\
\hline
\end{tabular}

Table 8. Partial decay widths for $h_{2} \rightarrow h_{1} h_{1}, h_{2} \rightarrow h_{1} h_{1} h_{1}$ and $h_{2} \rightarrow h_{1} h_{1} h_{1} h_{1}$. Other details as in tables 1 and 2 .

\section{B Nonredundant complete set of integrated results}

In tables $9-12$, a nonredundant complete set of integrated results is given.

\begin{tabular}{|c|c|}
\hline \multicolumn{2}{|c|}{$\begin{array}{c}(\rightarrow H) \rightarrow W^{-} W^{+} \rightarrow \ell \bar{\nu} \bar{\ell}^{\prime} \nu^{\prime} \\
\mathrm{SM}, p p, \sqrt{s}=13 \mathrm{TeV}\end{array}$} \\
\hline$|\mathcal{M}|^{2}$ & $\sigma[\mathrm{fb}]$ \\
\hline $\mathrm{Sq}(\mathrm{C})$ & $27.616(7)$ \\
$\mathrm{Sq}(H)$ & $13.689(4)$ \\
$\mathrm{I}(H, \mathrm{C})$ & $-3.269(4)$ \\
\hline
\end{tabular}

Table 9. Cross sections for $g g(\rightarrow H) \rightarrow W^{-} W^{+} \rightarrow \ell \bar{\nu} \bar{\ell}^{\prime} \nu^{\prime}$ in $p p$ collisions at $\sqrt{s}=13 \mathrm{TeV}$ in the Standard Model. Mod-squared amplitude contributions are specified using the abbreviations defined in table 3. The selection cuts in (4.1) are applied. Cross sections are given for a single lepton flavour combination. The integration error is displayed in brackets. 


\begin{tabular}{|c|c|c|c|c|c|}
\hline & & $\begin{array}{r}g g(\rightarrow \\
1 \mathrm{H}\end{array}$ & $\begin{array}{l}\left.\left.h_{1}, h_{2}\right\}\right) \rightarrow W \\
{[\mathrm{fb}], p p, \sqrt{s}=} \\
\mathrm{M} \text { (see tables }\end{array}$ & $\begin{array}{l}W^{+} \rightarrow \ell \bar{\nu} \bar{\ell}^{\prime} \nu^{\prime} \\
13 \mathrm{TeV} \\
1 \text { and } 3)\end{array}$ & \\
\hline$|M|^{2}$ & $\theta$ & & & ${ }_{2}[\mathrm{GeV}]$ & \\
\hline & & 700 & 1000 & 1500 & 3000 \\
\hline $\mathrm{Se}(C)$ & $\theta_{1}$ & $27.616(7)$ & $27.616(7)$ & $27.616(7)$ & $27.616(7)$ \\
\hline $\mathrm{NQ}(\mathrm{C})$ & $\theta_{2}$ & $27.616(7)$ & $27.616(7)$ & $27.616(7)$ & $27.616(7)$ \\
\hline $\mathrm{S}_{c}\left(h_{1}\right)$ & $\theta_{1}$ & $13.048(4)$ & $13.048(4)$ & $13.393(4)$ & $13.619(4)$ \\
\hline Sq( $\left(l_{1}\right)$ & $\theta_{2}$ & $11.541(4)$ & $11.541(4)$ & $12.697(4)$ & $13.436(4)$ \\
\hline $\mathrm{Sa}\left(h_{h}\right)$ & $\theta_{1}$ & $0.07810(2)$ & $0.010824(2)$ & $0.00027818(5)$ & $5.3026(9) \times 10^{-7}$ \\
\hline $\mathrm{Sq}\left(n_{2}\right)$ & $\theta_{2}$ & $0.27776(5)$ & $0.035182(6)$ & $0.0008885(2)$ & $2.3605(4) \times 10^{-6}$ \\
\hline$J(h-b)$ & $\theta_{1}$ & $-0.03697(4)$ & $-0.02704(2)$ & $-0.006028(2)$ & $-0.00032061(7)$ \\
\hline $1\left(n_{1}, n_{2}\right)$ & $\theta_{2}$ & $-0.1041(1)$ & $-0.07363(3)$ & $-0.017115(5)$ & $-0.0010893(3)$ \\
\hline$C)$ & $\theta_{1}$ & $-3.132(3)$ & $-3.132(3)$ & $-3.205(4)$ & $-3.251(4)$ \\
\hline $1\left(n_{1}, \mathrm{C}\right)$ & $\theta_{2}$ & $-2.796(3)$ & $-2.796(3)$ & $-3.051(2)$ & $-3.221(4)$ \\
\hline $\mathrm{J}(h, C)$ & $\theta_{1}$ & $0.05478(5)$ & $0.03401(2)$ & $0.006963(3)$ & $0.00035468(8)$ \\
\hline $1\left(n_{2}, \circlearrowright\right)$ & $\theta_{2}$ & $0.1765(2)$ & $0.10678(4)$ & $0.021519(6)$ & $0.0012430(3)$ \\
\hline
\end{tabular}

Table 10. Cross sections for $g g\left(\rightarrow\left\{h_{1}, h_{2}\right\}\right) \rightarrow W^{-} W^{+} \rightarrow \ell \bar{\nu} \bar{\ell}^{\prime} \nu^{\prime}$ in $p p$ collisions at $\sqrt{s}=13 \mathrm{TeV}$ in the 1-Higgs-Singlet Extension of the SM with $M_{h_{1}}=125 \mathrm{GeV}, M_{h_{2}}=700,1000,1500,3000 \mathrm{GeV}$ and mixing angles $\theta_{1}$ and $\theta_{2}$ (see table 1). Other details as in table 9 .

\begin{tabular}{|c|c|}
\hline \multicolumn{2}{|c|}{$\begin{array}{c}g g(\rightarrow H) \rightarrow t \bar{t} \rightarrow b \bar{b} \ell \bar{\nu} \bar{\ell}^{\prime} \nu^{\prime} \\
\mathrm{SM}, p p, \sqrt{s}=13 \mathrm{TeV}\end{array}$} \\
\hline$|\mathcal{M}|^{2}$ & $\sigma[\mathrm{fb}]$ \\
\hline $\mathrm{Sq}(\mathrm{C})$ & $2535.5(6)$ \\
\hline $\mathrm{Sq}(H)$ & $0.13367(4)$ \\
\hline $\mathrm{I}(H, \mathrm{C})$ & $-5.117(2)$ \\
\hline $\mathrm{I}\left(H, \mathrm{C}_{\circlearrowleft}\right)$ & $15.967(5)$ \\
\hline
\end{tabular}

Table 11. Cross sections for $g g(\rightarrow H) \rightarrow t \bar{t} \rightarrow b \bar{b} \ell \bar{\nu} \bar{\ell}^{\prime} \nu^{\prime}$ in $p p$ collisions at $\sqrt{s}=13 \mathrm{TeV}$ in the Standard Model. Other details as in table 9. 


\begin{tabular}{|c|c|c|c|c|c|}
\hline \multicolumn{6}{|c|}{$\begin{array}{c}g g\left(\rightarrow\left\{h_{1}, h_{2}\right\}\right) \rightarrow t \bar{t} \rightarrow b \bar{b} \ell \bar{\nu} \bar{\ell}^{\prime} \nu^{\prime} \\
\sigma[\mathrm{fb}], p p, \sqrt{s}=13 \mathrm{TeV} \\
1 \mathrm{HSM}(\text { see tables } 1 \text { and } 3)\end{array}$} \\
\hline \multirow{2}{*}{$|\mathcal{M}|^{2}$} & \multirow{2}{*}{$\theta$} & \multicolumn{4}{|c|}{$M_{h_{2}}[\mathrm{GeV}]$} \\
\hline & & 700 & 1000 & 1500 & 3000 \\
\hline \multirow{2}{*}{$\mathrm{Sq}(\mathrm{C})$} & $\theta_{1}$ & $2535.2(6)$ & $2535.2(6)$ & $2535.2(6)$ & $2535.2(6)$ \\
\hline & $\theta_{2}$ & $2535.2(6)$ & $2535.2(6)$ & $2535.2(6)$ & $2535.2(6)$ \\
\hline \multirow{2}{*}{$\operatorname{Sq}\left(h_{1}\right)$} & $\theta_{1}$ & $0.12228(4)$ & $0.12228(4)$ & $0.12827(4)$ & $0.13233(4)$ \\
\hline & $\theta_{2}$ & $0.09734(3)$ & $0.09734(3)$ & $0.11631(3)$ & $0.12911(4)$ \\
\hline \multirow{2}{*}{$\operatorname{Sq}\left(h_{2}\right)$} & $\theta_{1}$ & $0.015207(4)$ & $0.0012148(4)$ & $1.2910(4) \times 10^{-5}$ & $7.858(3) \times 10^{-9}$ \\
\hline & $\theta_{2}$ & $0.05395(2)$ & $0.004151(2)$ & $5.566(2) \times 10^{-5}$ & $8.503(3) \times 10^{-8}$ \\
\hline \multirow{2}{*}{$\mathrm{I}\left(h_{1}, h_{2}\right)$} & $\theta_{1}$ & $-0.009140(9)$ & $-0.005293(3)$ & $-0.0009301(3)$ & $-4.656(2) \times 10^{-5}$ \\
\hline & $\theta_{2}$ & $-0.02553(2)$ & $-0.014530(5)$ & $-0.0027239(8)$ & $-0.00015904(5)$ \\
\hline \multirow{2}{*}{$\mathrm{I}\left(h_{1}, \mathrm{C}\right)$} & $\theta_{1}$ & $-4.893(2)$ & $-4.893(2)$ & $-5.011(2)$ & $-5.090(2)$ \\
\hline & $\theta_{2}$ & $-4.365(2)$ & $-4.365(2)$ & $-4.772(2)$ & $-5.027(2)$ \\
\hline \multirow{2}{*}{$\mathrm{I}\left(h_{2}, \mathrm{C}\right)$} & $\theta_{1}$ & $-0.01350(2)$ & $0.03602(3)$ & $0.009967(4)$ & $0.0006248(2)$ \\
\hline & $\theta_{2}$ & $-0.07772(8)$ & $0.08367(9)$ & $0.02335(1)$ & $0.0019221(6)$ \\
\hline \multirow{2}{*}{$\mathrm{I}\left(h_{1}, \mathrm{C}_{\circlearrowleft}\right)$} & $\theta_{1}$ & $15.277(5)$ & $15.277(5)$ & $15.644(5)$ & $15.890(5)$ \\
\hline & $\theta_{2}$ & $13.629(4)$ & $13.629(4)$ & $14.898(5)$ & $15.695(5)$ \\
\hline \multirow{2}{*}{$\mathrm{I}\left(h_{2}, \mathrm{C}_{\circlearrowleft}\right)$} & $\theta_{1}$ & $0.7040(6)$ & $0.06697(7)$ & $-0.01183(2)$ & $-0.0013431(7)$ \\
\hline & $\theta_{2}$ & $2.485(2)$ & $0.4122(4)$ & $0.01486(2)$ & $-0.003009(3)$ \\
\hline
\end{tabular}

Table 12. Cross sections for $g g\left(\rightarrow\left\{h_{1}, h_{2}\right\}\right) \rightarrow t \bar{t} \rightarrow b \bar{b} \ell \bar{\nu} \bar{\ell}^{\prime} \nu^{\prime}$ in $p p$ collisions at $\sqrt{s}=13 \mathrm{TeV}$ in the 1HSM. Other details as in tables 9 and 10 .

\section{Standard Model distributions}

Invariant mass and transverse invariant mass distributions for $W W$ and $t \bar{t}$ production in the SM are displayed in figures $26-29$. 


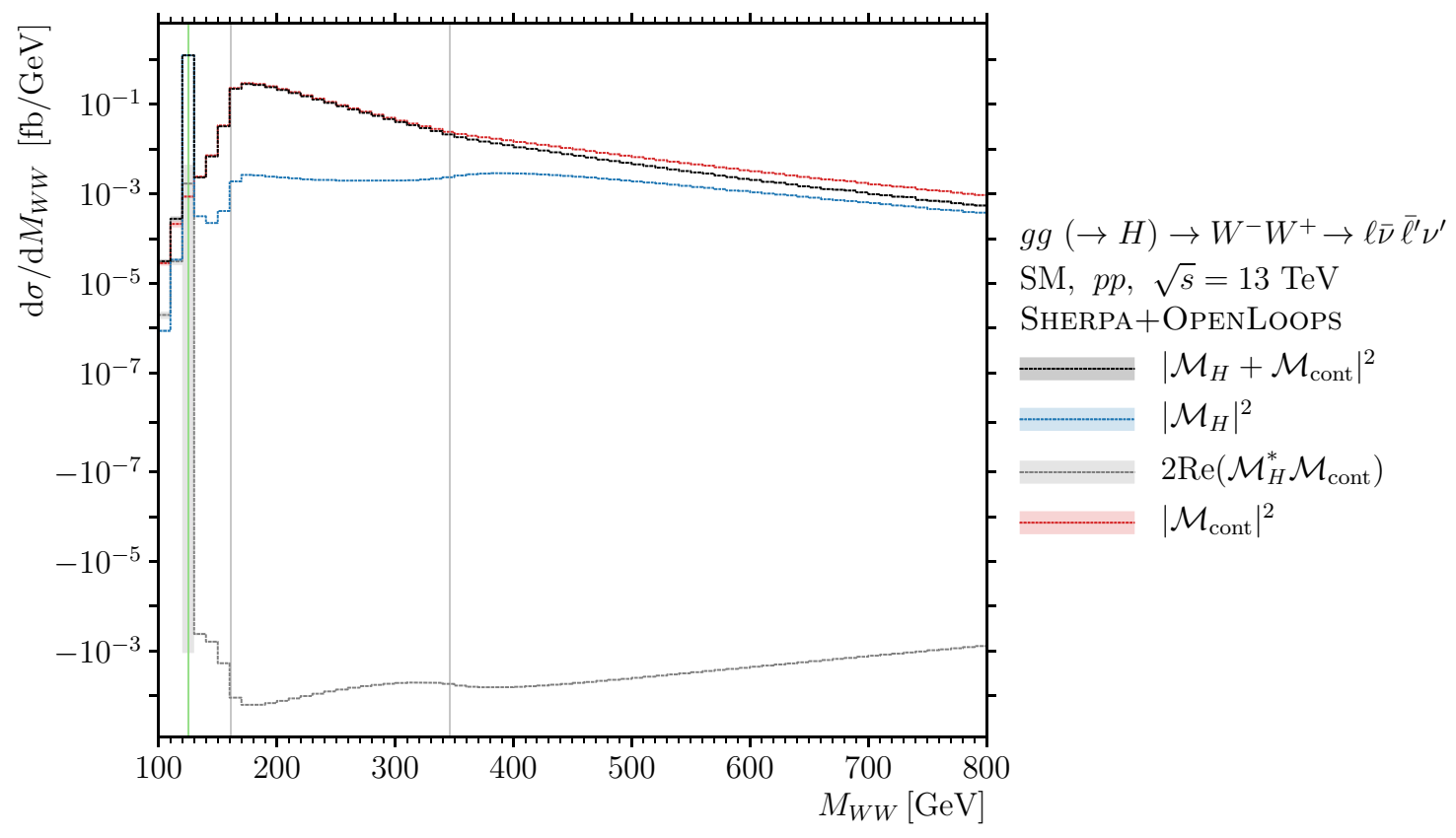

Figure 26. $\quad M_{W W}$ distributions for the process $g g(\rightarrow H) \rightarrow W^{-} W^{+} \rightarrow \ell \bar{\nu} \bar{\ell}^{\prime} \nu^{\prime}$ in the SM including its interference with the background in $p p$ collisions at $\sqrt{s}=13 \mathrm{TeV}$. Other details as in figure 4 .

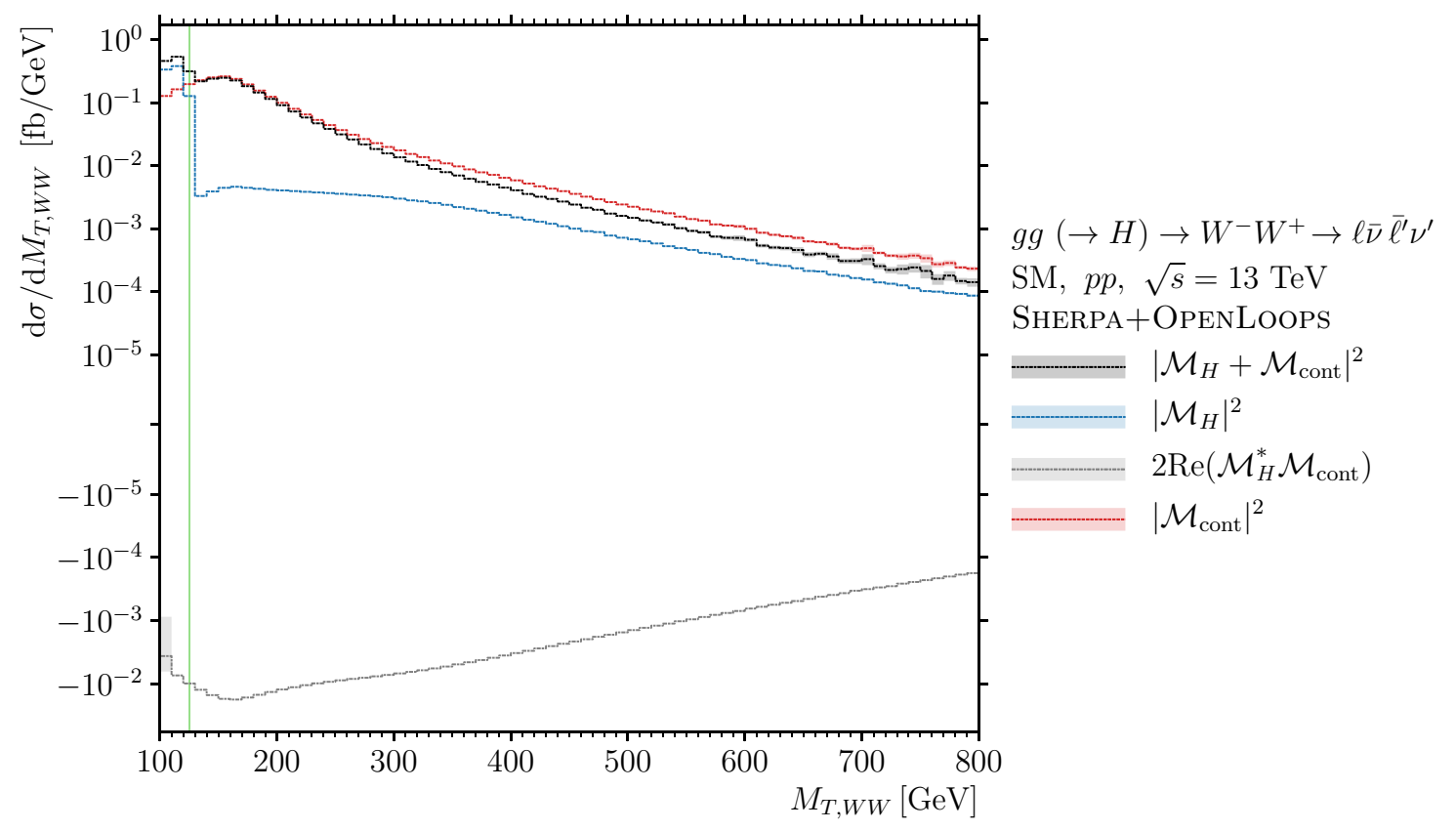

Figure 27. $M_{T, W W}$ distributions for the process $g g(\rightarrow H) \rightarrow W^{-} W^{+} \rightarrow \ell \bar{\nu} \bar{\ell}^{\prime} \nu^{\prime}$ in the SM including its interference with the background in $p p$ collisions at $\sqrt{s}=13 \mathrm{TeV}$. Other details as in figure 4 . 


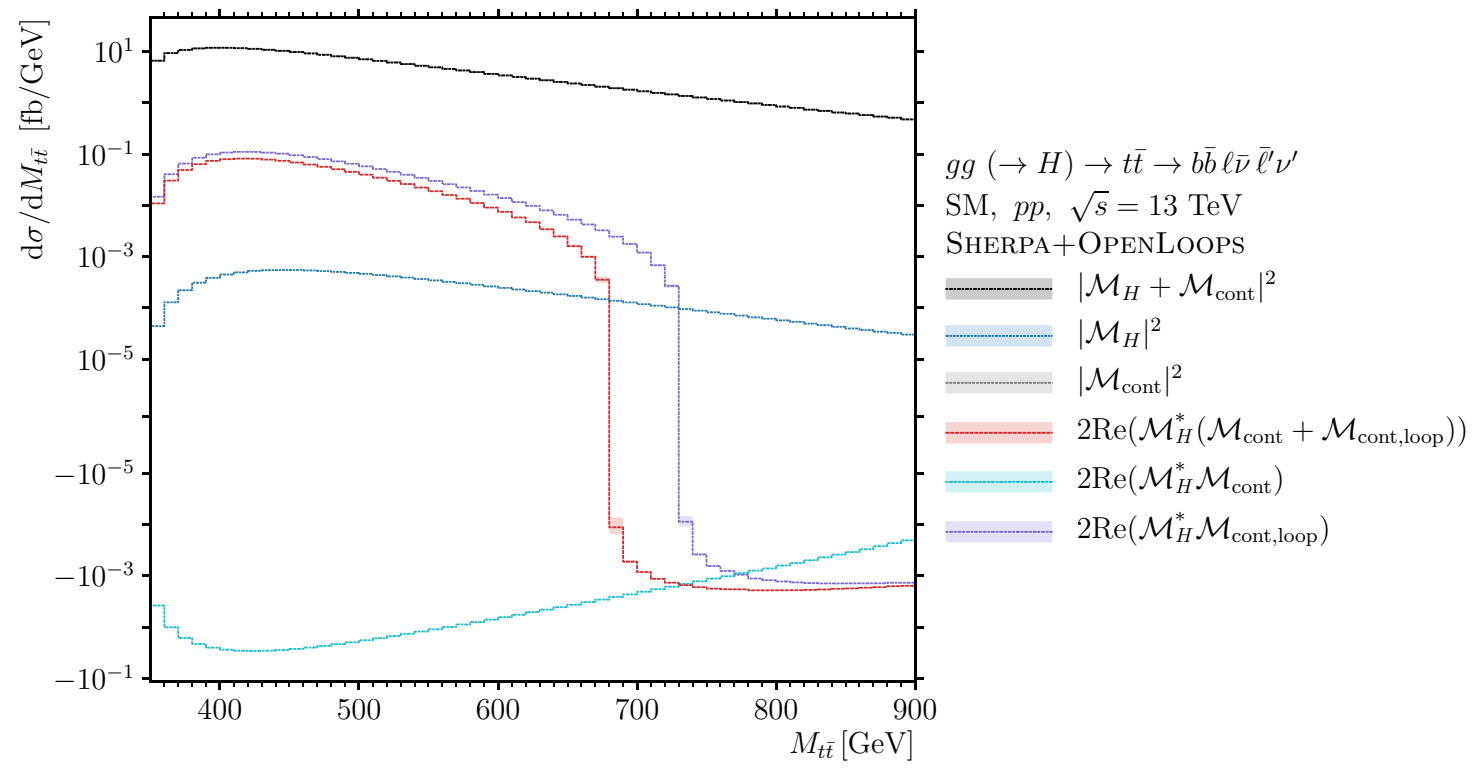

Figure 28. $M_{t \bar{t}}$ distributions for the process $g g(\rightarrow H) \rightarrow t \bar{t} \rightarrow b \bar{b} \ell \bar{\nu} \bar{\ell}^{\prime} \nu^{\prime}$ in the SM including its interference with the background in $p p$ collisions at $\sqrt{s}=13 \mathrm{TeV}$. Other details as in figure 4.

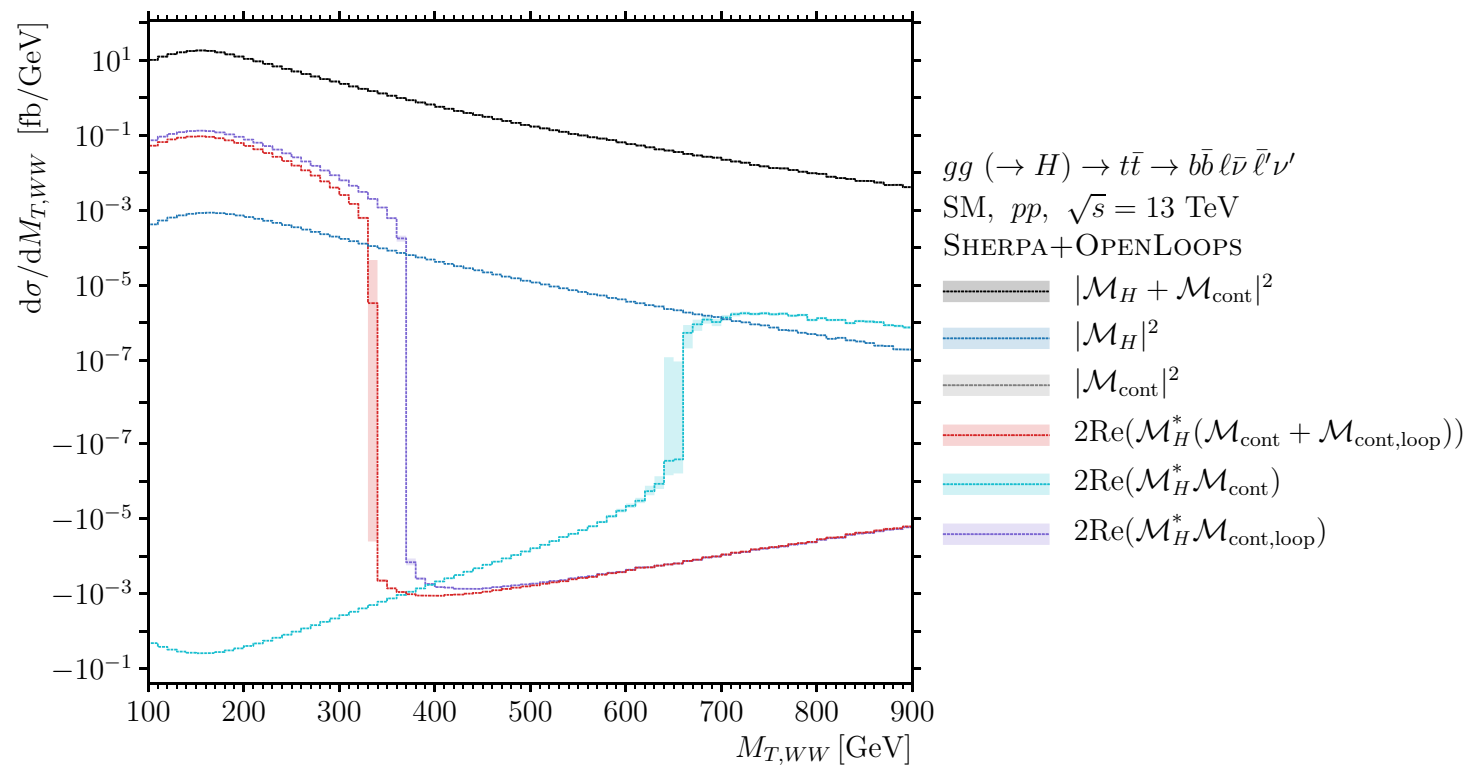

Figure 29. $\quad M_{T, W W}$ distributions for the process $g g(\rightarrow H) \rightarrow t \bar{t} \rightarrow b \bar{b} \ell \bar{\nu} \bar{\ell}^{\prime} \nu^{\prime}$ in the SM including its interference with the background in $p p$ collisions at $\sqrt{s}=13 \mathrm{TeV}$. Other details as in figure 4 . 
Open Access. This article is distributed under the terms of the Creative Commons Attribution License (CC-BY 4.0), which permits any use, distribution and reproduction in any medium, provided the original author(s) and source are credited.

\section{References}

[1] ATLAS collaboration, Observation of a new particle in the search for the Standard Model Higgs boson with the ATLAS detector at the LHC, Phys. Lett. B 716 (2012) 1 [arXiv: 1207.7214] [INSPIRE].

[2] CMS collaboration, Observation of a New Boson at a Mass of $125 \mathrm{GeV}$ with the CMS Experiment at the LHC, Phys. Lett. B 716 (2012) 30 [arXiv:1207.7235] [INSPIRE].

[3] P.W. Higgs, Broken symmetries, massless particles and gauge fields, Phys. Lett. 12 (1964) 132 [INSPIRE].

[4] P.W. Higgs, Broken Symmetries and the Masses of Gauge Bosons, Phys. Rev. Lett. 13 (1964) 508 [INSPIRE].

[5] P.W. Higgs, Spontaneous Symmetry Breakdown without Massless Bosons, Phys. Rev. 145 (1966) 1156 [INSPIRE].

[6] F. Englert and R. Brout, Broken Symmetry and the Mass of Gauge Vector Mesons, Phys. Rev. Lett. 13 (1964) 321 [INSPIRE].

[7] G.S. Guralnik, C.R. Hagen and T.W.B. Kibble, Global Conservation Laws and Massless Particles, Phys. Rev. Lett. 13 (1964) 585 [INSPIRE].

[8] T. Binoth and J.J. van der Bij, Influence of strongly coupled, hidden scalars on Higgs signals, Z. Phys. C 75 (1997) 17 [hep-ph/9608245] [INSPIRE].

[9] R.M. Schabinger and J.D. Wells, A Minimal spontaneously broken hidden sector and its impact on Higgs boson physics at the large hadron collider, Phys. Rev. D 72 (2005) 093007 [hep-ph/0509209] [INSPIRE].

[10] B. Patt and F. Wilczek, Higgs-field portal into hidden sectors, hep-ph/0605188 [INSPIRE].

[11] M. Bowen, Y. Cui and J.D. Wells, Narrow trans-TeV Higgs bosons and $H \rightarrow$ hh decays: Two LHC search paths for a hidden sector Higgs boson, JHEP 03 (2007) 036 [hep-ph/0701035] [INSPIRE].

[12] V. Barger, P. Langacker, M. McCaskey, M.J. Ramsey-Musolf and G. Shaughnessy, LHC Phenomenology of an Extended Standard Model with a Real Scalar Singlet, Phys. Rev. D 77 (2008) 035005 [arXiv:0706.4311] [INSPIRE].

[13] V. Barger, P. Langacker, M. McCaskey, M. Ramsey-Musolf and G. Shaughnessy, Complex Singlet Extension of the Standard Model, Phys. Rev. D 79 (2009) 015018 [arXiv:0811. 0393] [INSPIRE].

[14] G. Bhattacharyya, G.C. Branco and S. Nandi, Universal Doublet-Singlet Higgs Couplings and phenomenology at the CERN Large Hadron Collider, Phys. Rev. D 77 (2008) 117701 [arXiv: 0712.2693] [INSPIRE].

[15] S. Dawson and W. Yan, Hiding the Higgs Boson with Multiple Scalars, Phys. Rev. D 79 (2009) 095002 [arXiv:0904.2005] [INSPIRE]. 
[16] S. Bock, R. Lafaye, T. Plehn, M. Rauch, D. Zerwas and P.M. Zerwas, Measuring Hidden Higgs and Strongly-Interacting Higgs Scenarios, Phys. Lett. B 694 (2011) 44 [arXiv: 1007.2645] [INSPIRE].

[17] P.J. Fox, D. Tucker-Smith and N. Weiner, Higgs friends and counterfeits at hadron colliders, JHEP 06 (2011) 127 [arXiv: 1104.5450] [INSPIRE].

[18] C. Englert, T. Plehn, D. Zerwas and P.M. Zerwas, Exploring the Higgs portal, Phys. Lett. B 703 (2011) 298 [arXiv: 1106.3097] [INSPIRE].

[19] C. Englert, J. Jaeckel, E. Re and M. Spannowsky, Evasive Higgs Maneuvers at the LHC, Phys. Rev. D 85 (2012) 035008 [arXiv:1111.1719] [INSPIRE].

[20] B. Batell, S. Gori and L.-T. Wang, Exploring the Higgs Portal with 10/fb at the LHC, JHEP 06 (2012) 172 [arXiv:1112.5180] [INSPIRE].

[21] C. Englert, T. Plehn, M. Rauch, D. Zerwas and P.M. Zerwas, LHC: Standard Higgs and Hidden Higgs, Phys. Lett. B 707 (2012) 512 [arXiv:1112.3007] [InSPIRE].

[22] R.S. Gupta and J.D. Wells, Higgs boson search significance deformations due to mixed-in scalars, Phys. Lett. B 710 (2012) 154 [arXiv:1110.0824] [INSPIRE].

[23] M.J. Dolan, C. Englert and M. Spannowsky, New Physics in LHC Higgs boson pair production, Phys. Rev. D 87 (2013) 055002 [arXiv: 1210.8166] [INSPIRE].

[24] B. Batell, D. McKeen and M. Pospelov, Singlet Neighbors of the Higgs Boson, JHEP 10 (2012) 104 [arXiv:1207.6252] [INSPIRE].

[25] J.M. No and M. Ramsey-Musolf, Probing the Higgs Portal at the LHC Through Resonant di-Higgs Production, Phys. Rev. D 89 (2014) 095031 [arXiv:1310.6035] [inSPIRE].

[26] R. Coimbra, M.O.P. Sampaio and R. Santos, ScannerS: Constraining the phase diagram of a complex scalar singlet at the LHC, Eur. Phys. J. C 73 (2013) 2428 [arXiv:1301.2599] [INSPIRE].

[27] S. Profumo, M.J. Ramsey-Musolf, C.L. Wainwright and P. Winslow, Singlet-catalyzed electroweak phase transitions and precision Higgs boson studies, Phys. Rev. D 91 (2015) 035018 [arXiv: 1407.5342] [INSPIRE].

[28] H.E. Logan, Hiding a Higgs width enhancement from off-shell $g g\left(\rightarrow h^{*}\right) \rightarrow Z Z$ measurements, Phys. Rev. D 92 (2015) 075038 [arXiv:1412.7577] [INSPIRE].

[29] C.-Y. Chen, S. Dawson and I.M. Lewis, Exploring resonant di-Higgs boson production in the Higgs singlet model, Phys. Rev. D 91 (2015) 035015 [arXiv:1410.5488] [INSPIRE].

[30] R. Costa, A.P. Morais, M.O.P. Sampaio and R. Santos, Two-loop stability of a complex singlet extended Standard Model, Phys. Rev. D 92 (2015) 025024 [arXiv:1411.4048] [INSPIRE].

[31] A. Falkowski, C. Gross and O. Lebedev, A second Higgs from the Higgs portal, JHEP 05 (2015) 057 [arXiv: 1502.01361] [INSPIRE].

[32] V. Martn Lozano, J.M. Moreno and C.B. Park, Resonant Higgs boson pair production in the $h h \rightarrow b \bar{b} W W \rightarrow b \bar{b} \ell^{+} \nu \ell^{-} \bar{\nu}$ decay channel, JHEP 08 (2015) 004 [arXiv: 1501. 03799] [INSPIRE].

[33] S. Kanemura, M. Kikuchi and K. Yagyu, Radiative corrections to the Higgs boson couplings in the model with an additional real singlet scalar field, Nucl. Phys. B 907 (2016) 286 [arXiv: 1511.06211] [INSPIRE]. 
[34] S. Kanemura, M. Kikuchi and K. Yagyu, One-loop corrections to the Higgs self-couplings in the singlet extension, Nucl. Phys. B 917 (2017) 154 [arXiv:1608.01582] [INSPIRE].

[35] S. Kanemura, M. Kikuchi, K. Sakurai and K. Yagyu, H-COUP: a program for one-loop corrected Higgs boson couplings in non-minimal Higgs sectors, Comput. Phys. Commun. 233 (2018) 134 [arXiv: 1710.04603] [INSPIRE].

[36] I.M. Lewis and M. Sullivan, Benchmarks for Double Higgs Production in the Singlet Extended Standard Model at the LHC, Phys. Rev. D 96 (2017) 035037 [arXiv:1701.08774] [InSPIRE].

[37] J.A. Casas, D.G. Cerdeño, J.M. Moreno and J. Quilis, Reopening the Higgs portal for single scalar dark matter, JHEP 05 (2017) 036 [arXiv:1701.08134] [INSPIRE].

[38] L. Altenkamp, M. Boggia and S. Dittmaier, Precision calculations for $h \rightarrow W W / Z Z \rightarrow 4$ fermions in a Singlet Extension of the Standard Model with Prophecy 4 f, JHEP 04 (2018) 062 [arXiv: 1801.07291] [INSPIRE].

[39] G.M. Pruna and T. Robens, Higgs singlet extension parameter space in the light of the LHC discovery, Phys. Rev. D 88 (2013) 115012 [arXiv:1303.1150] [InSPIRE].

[40] T. Robens and T. Stefaniak, Status of the Higgs Singlet Extension of the Standard Model after LHC Run 1, Eur. Phys. J. C 75 (2015) 104 [arXiv:1501.02234] [INSPIRE].

[41] T. Robens and T. Stefaniak, LHC Benchmark Scenarios for the Real Higgs Singlet Extension of the Standard Model, Eur. Phys. J. C 76 (2016) 268 [arXiv:1601.07880] [inSPIRE].

[42] A. Ilnicka, T. Robens and T. Stefaniak, Constraining Extended Scalar Sectors at the LHC and beyond, Mod. Phys. Lett. A 33 (2018) 1830007 [arXiv: 1803.03594] [INSPIRE].

[43] CMS collaboration, Search for a standard-model-like Higgs boson with a mass in the range 145 to $1000 \mathrm{GeV}$ at the LHC, Eur. Phys. J. C 73 (2013) 2469 [arXiv:1304.0213] [INSPIRE].

[44] ATLAS collaboration, Search for a high-mass Higgs boson decaying to a $W$ boson pair in pp collisions at $\sqrt{s}=8 \mathrm{TeV}$ with the ATLAS detector, JHEP 01 (2016) 032 [arXiv: 1509.00389] [INSPIRE].

[45] CMS collaboration, Search for a Higgs boson in the mass range from 145 to $1000 \mathrm{GeV}$ decaying to a pair of $W$ or $Z$ bosons, JHEP 10 (2015) 144 [arXiv: 1504.00936] [INSPIRE].

[46] ATLAS collaboration, Searches for heavy diboson resonances in pp collisions at $\sqrt{s}=13 \mathrm{TeV}$ with the ATLAS detector, JHEP 09 (2016) 173 [arXiv:1606.04833] [INSPIRE].

[47] ATLAS collaboration, Search for diboson resonances with boson-tagged jets in pp collisions at $\sqrt{s}=13 \mathrm{TeV}$ with the ATLAS detector, Phys. Lett. B 777 (2018) 91 [arXiv:1708.04445] [INSPIRE].

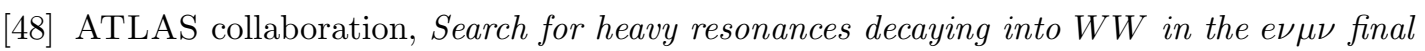
state in pp collisions at $\sqrt{s}=13 \mathrm{TeV}$ with the ATLAS detector, Eur. Phys. J. C 78 (2018) 24 [arXiv: 1710.01123 ] [INSPIRE].

[49] ATLAS collaboration, Search for $W W / W Z$ resonance production in $\ell \nu q q$ final states in $p p$ collisions at $\sqrt{s}=13 \mathrm{TeV}$ with the ATLAS detector, JHEP 03 (2018) 042 [arXiv: 1710.07235] [INSPIRE].

[50] ATLAS collaboration, Search for Heavy Higgs Bosons A/H Decaying to a Top Quark Pair in pp Collisions at $\sqrt{s}=8 T e V$ with the ATLAS Detector, Phys. Rev. Lett. 119 (2017) 191803 [arXiv:1707.06025] [INSPIRE]. 
[51] ATLAS collaboration, Search for heavy particles decaying into top-quark pairs using lepton-plus-jets events in proton-proton collisions at $\sqrt{s}=13$ TeV with the ATLAS detector, Eur. Phys. J. C 78 (2018) 565 [arXiv: 1804.10823] [inSPIRE].

[52] N. Kauer and G. Passarino, Inadequacy of zero-width approximation for a light Higgs boson signal, JHEP 08 (2012) 116 [arXiv:1206.4803] [INSPIRE].

[53] E. Maina, Interference effects in Heavy Higgs production via gluon fusion in the Singlet Extension of the Standard Model, JHEP 06 (2015) 004 [arXiv:1501.02139] [INSPIRE].

[54] N. Kauer and C. O'Brien, Heavy Higgs signal-background interference in $g g \rightarrow V V$ in the Standard Model plus real singlet, Eur. Phys. J. C 75 (2015) 374 [arXiv:1502.04113] [INSPIRE].

[55] C. Englert, I. Low and M. Spannowsky, On-shell interference effects in Higgs boson final states, Phys. Rev. D 91 (2015) 074029 [arXiv:1502.04678] [INSPIRE].

[56] N. Greiner, S. Liebler and G. Weiglein, Interference contributions to gluon initiated heavy Higgs production in the Two-Higgs-Doublet Model, Eur. Phys. J. C 76 (2016) 118 [arXiv: 1512.07232] [INSPIRE].

[57] C. Englert, Y. Soreq and M. Spannowsky, Off-Shell Higgs Coupling Measurements in BSM scenarios, JHEP 05 (2015) 145 [arXiv: 1410.5440] [INSPIRE].

[58] S. Liebler, G. Moortgat-Pick and G. Weiglein, Off-shell effects in Higgs processes at a linear collider and implications for the LHC, JHEP 06 (2015) 093 [arXiv: 1502.07970] [INSPIRE].

[59] A. Ballestrero and E. Maina, Interference Effects in Higgs production through Vector Boson Fusion in the Standard Model and its Singlet Extension, JHEP 01 (2016) 045 [arXiv: 1506. 02257] [INSPIRE].

[60] D. Dicus, A. Stange and S. Willenbrock, Higgs decay to top quarks at hadron colliders, Phys. Lett. B 333 (1994) 126 [hep-ph/9404359] [INSPIRE].

[61] W. Bernreuther, P. Galler, C. Mellein, Z.G. Si and P. Uwer, Production of heavy Higgs bosons and decay into top quarks at the LHC, Phys. Rev. D 93 (2016) 034032 [arXiv: 1511.05584] [INSPIRE].

[62] B. Hespel, F. Maltoni and E. Vryonidou, Signal background interference effects in heavy scalar production and decay to a top-anti-top pair, JHEP 10 (2016) 016 [arXiv:1606.04149] [INSPIRE].

[63] W. Bernreuther, P. Galler, Z.-G. Si and P. Uwer, Production of heavy Higgs bosons and decay into top quarks at the LHC. II: Top-quark polarization and spin correlation effects, Phys. Rev. D 95 (2017) 095012 [arXiv:1702.06063] [INSPIRE].

[64] M. Carena and Z. Liu, Challenges and opportunities for heavy scalar searches in the t $\bar{t}$ channel at the LHC, JHEP 11 (2016) 159 [arXiv:1608.07282] [INSPIRE].

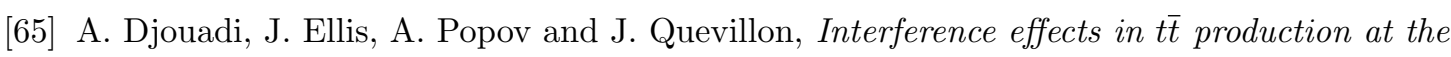
LHC as a window on new physics, JHEP 03 (2019) 119 [arXiv:1901.03417] [INSPIRE].

[66] L.J. Dixon and M.S. Siu, Resonance continuum interference in the diphoton Higgs signal at the LHC, Phys. Rev. Lett. 90 (2003) 252001 [hep-ph/0302233] [INSPIRE].

[67] N. Kauer, C. O'Brien and E. Vryonidou, Interference effects for $H \rightarrow W W \rightarrow \ell \nu q \bar{q}^{\prime}$ and $H \rightarrow Z Z \rightarrow \bar{\ell} q \bar{q}$ searches in gluon fusion at the LHC, JHEP 10 (2015) 074 [arXiv: 1506.01694] [INSPIRE]. 
[68] J. Campbell, M. Carena, R. Harnik and Z. Liu, Interference in the gg $\rightarrow h \rightarrow \gamma \gamma$ On-Shell Rate and the Higgs Boson Total Width, Phys. Rev. Lett. 119 (2017) 181801 [arXiv: 1704.08259] [INSPIRE].

[69] U. Haisch and G. Polesello, Searching for heavy Higgs bosons in the $t \bar{t} Z$ and $t b W$ final states, JHEP 09 (2018) 151 [arXiv: 1807.07734] [INSPIRE].

[70] J. Baglio, S. Dawson and I.M. Lewis, NLO effects in EFT fits to $W^{+} W^{-}$production at the LHC, Phys. Rev. D 99 (2019) 035029 [arXiv: 1812.00214] [InSPIRE].

[71] LHC Higgs Cross Section Working Group collaboration, Handbook of LHC Higgs Cross Sections: 3. Higgs Properties, arXiv:1307.1347 [INSPIRE].

[72] LHC Higgs Cross Section Working Group collaboration, Handbook of LHC Higgs Cross Sections: 4. Deciphering the Nature of the Higgs Sector, arXiv:1610.07922 [INSPIRE].

[73] J. Butterworth et al., PDF4LHC recommendations for LHC Run II, J. Phys. G 43 (2016) 023001 [arXiv: 1510.03865] [INSPIRE].

[74] N. Kauer, Signal-background interference in $g g \rightarrow H \rightarrow V V$, arXiv:1201.1667 [INSPIRE].

[75] A. Denner and S. Dittmaier, The Complex-mass scheme for perturbative calculations with unstable particles, Nucl. Phys. Proc. Suppl. 160 (2006) 22 [hep-ph/0605312] [INSPIRE].

[76] A. Denner, S. Dittmaier, M. Roth and D. Wackeroth, Predictions for all processes $e^{+} e^{-} \rightarrow 4$ fermions + gamma, Nucl. Phys. B 560 (1999) 33 [hep-ph/9904472] [INSPIRE].

[77] A. Djouadi, J. Kalinowski and M. Spira, HDECAY: A Program for Higgs boson decays in the standard model and its supersymmetric extension, Comput. Phys. Commun. 108 (1998) 56 [hep-ph/9704448] [INSPIRE].

[78] A. Djouadi, J. Kalinowski, M. Muehlleitner and M. Spira, HDECAY: Twenty $y_{++}$years after, Comput. Phys. Commun. 238 (2019) 214 [arXiv:1801.09506] [InSPIRE].

[79] A. Bredenstein, A. Denner, S. Dittmaier and M.M. Weber, Precise predictions for the Higgs-boson decay $H \rightarrow W W / Z Z \rightarrow 4$ leptons, Phys. Rev. D 74 (2006) 013004 [hep-ph/0604011] [INSPIRE].

[80] A. Bredenstein, A. Denner, S. Dittmaier and M.M. Weber, Precision calculations for the Higgs decays $H \rightarrow Z Z / W W \rightarrow 4$ leptons, Nucl. Phys. Proc. Suppl. 160 (2006) 131 [hep-ph/0607060] [INSPIRE].

[81] A. Bredenstein, A. Denner, S. Dittmaier and M.M. Weber, Radiative corrections to the semileptonic and hadronic Higgs-boson decays $H \rightarrow W W / Z Z \rightarrow 4$ fermions, JHEP 02 (2007) 080 [hep-ph/0611234] [INSPIRE].

[82] A. Alloul, N.D. Christensen, C. Degrande, C. Duhr and B. Fuks, FeynRules 2.0 - A complete toolbox for tree-level phenomenology, Comput. Phys. Commun. 185 (2014) 2250 [arXiv:1310.1921] [INSPIRE].

[83] N.D. Christensen and C. Duhr, FeynRules - Feynman rules made easy, Comput. Phys. Commun. 180 (2009) 1614 [arXiv:0806.4194] [INSPIRE].

[84] C. Degrande, C. Duhr, B. Fuks, D. Grellscheid, O. Mattelaer and T. Reiter, UFO - The Universal FeynRules Output, Comput. Phys. Commun. 183 (2012) 1201 [arXiv:1108.2040] [INSPIRE]. 
[85] J. Alwall et al., The automated computation of tree-level and next-to-leading order differential cross sections and their matching to parton shower simulations, JHEP 07 (2014) 079 [arXiv: 1405.0301] [INSPIRE].

[86] F. Cascioli, P. Maierhofer and S. Pozzorini, Scattering Amplitudes with Open Loops, Phys. Rev. Lett. 108 (2012) 111601 [arXiv:1111.5206] [INSPIRE].

[87] A. Denner, S. Dittmaier and L. Hofer, Collier: a fortran-based Complex One-Loop LIbrary in Extended Regularizations, Comput. Phys. Commun. 212 (2017) 220 [arXiv:1604.06792] [INSPIRE].

[88] T. Gleisberg et al., Event generation with SHERPA 1.1, JHEP 02 (2009) 007 [arXiv:0811.4622] [INSPIRE].

[89] F. Krauss, R. Kuhn and G. Soff, AMEGIC++ 1.0: A Matrix element generator in $\mathrm{C}++$, JHEP 02 (2002) 044 [hep-ph/0109036] [INSPIRE].

[90] A. Buckley et al., LHAPDF6: parton density access in the LHC precision era, Eur. Phys. J. C 75 (2015) 132 [arXiv: 1412.7420] [INSPIRE].

[91] A. Denner, S. Dittmaier, M. Roth and D. Wackeroth, Electroweak radiative corrections to $e^{+} e^{-} \rightarrow W W \rightarrow 4$ fermions in double pole approximation: The RACOONWW approach, Nucl. Phys. B 587 (2000) 67 [hep-ph/0006307] [INSPIRE].

[92] J.M. Campbell, R.K. Ellis, E. Furlan and R. Röntsch, Interference effects for Higgs boson mediated Z-pair plus jet production, Phys. Rev. D 90 (2014) 093008 [arXiv:1409.1897] [INSPIRE].

[93] V.S. Fadin, V.A. Khoze and A.D. Martin, How suppressed are the radiative interference effects in heavy instable particle production?, Phys. Lett. B 320 (1994) 141 [hep-ph/9309234] [INSPIRE].

[94] T. Kinoshita, Mass singularities of Feynman amplitudes, J. Math. Phys. 3 (1962) 650 [INSPIRE].

[95] T.D. Lee and M. Nauenberg, Degenerate Systems and Mass Singularities, Phys. Rev. 133 (1964) B1549 [INSPIRE].

[96] T. Binoth et al., A Proposal for a Standard Interface between Monte Carlo Tools and One-Loop Programs, Comput. Phys. Commun. 181 (2010) 1612 [arXiv:1001.1307] [INSPIRE].

[97] M. Grazzini, S. Kallweit, M. Wiesemann and J.Y. Yook, ZZ production at the LHC: NLO QCD corrections to the loop-induced gluon fusion channel, JHEP 03 (2019) 070 [arXiv: 1811.09593] [INSPIRE].

[98] ATLAS collaboration, Measurements of gluon-gluon fusion and vector-boson fusion Higgs boson production cross-sections in the $H \rightarrow W W^{*} \rightarrow e \nu \mu \nu$ decay channel in pp collisions at $\sqrt{s}=13 \mathrm{TeV}$ with the ATLAS detector, Phys. Lett. B 789 (2019) 508 [arXiv:1808.09054] [INSPIRE].

[99] CMS collaboration, Measurements of the Higgs boson width and anomalous HVV couplings from on-shell and off-shell production in the four-lepton final state, Phys. Rev. D 99 (2019) 112003 [arXiv: 1901.00174] [INSPIRE].

[100] http://users.hepforge.org/ nkauer/arXiv/plots_08May2019.pdf. 
[101] M. Dittmar and H.K. Dreiner, How to find a Higgs boson with a mass between 155-GeV-180-GeV at the LHC, Phys. Rev. D 55 (1997) 167 [hep-ph/9608317] [INSPIRE].

[102] A. Buckley et al., Rivet user manual, Comput. Phys. Commun. 184 (2013) 2803 [arXiv: 1003.0694] [INSPIRE].

[103] D. Binosi and L. Theussl, JaxoDraw: A Graphical user interface for drawing Feynman diagrams, Comput. Phys. Commun. 161 (2004) 76 [hep-ph/0309015] [INSPIRE].

[104] J.D. Hunter, Matplotlib: A 2D Graphics Environment, Comput. Sci. Eng. 9 (2007) 90 [INSPIRE]. 